\title{
Sepsis 2017 Paris
}

Paris, France. September 11-13, 2017

Published: 11 September 2017

P1

Is Post-Sepsis Syndrome different from Post-Intensive Care (ICU) syndrome? A cohort and propensity score matched analysis

Kelly Thompson, Colman Taylor, Stephen Jan, Qiang Li, John Myburgh, Bala Venkatesh, Simon Finfer

The George Institute for Global Health, Newtown, NSW 2042, Australia Correspondence: Kelly Thompson (kthompson@georgeinstitute.org.au) Intensive Care Medicine Experimental 2017, 5(Suppl 1):P1

\section{Background}

Post-Sepsis Syndrome and Post-ICU Syndrome are terms recently adopted to characterise the health-related quality of life (QoL) issues affecting patients after sepsis or after treatment in an ICU.[1, 2] Whether these syndromes are different is not known.

Objectives

To determine QoL, late mortality, costs and healthcare resource use of ICU patients with sepsis compared to those without sepsis.

Methods

After obtaining ethical committee consent we analysed data of ICU patients with sepsis recruited to a randomised controlled trial in New South Wales, Australia. We conducted a cohort and propensity score matched (PSM) analysis using baseline variables including indices of severity of illness compared to ICU patients without sepsis. We assessed QoL using the EQ-5D-3 L at 6 months and mortality, costs (in $\$ A U$ ) and healthcare resource use at 2 years.

Results

QoL was assessed in 4975 patients: 1320 with sepsis and 3655 without. There were no significant differences in QoL. For the analysis of mortality, costs and healthcare resource use, 3442 patients were included: 905 patients with sepsis and 2537 without. At 2 years, patients with sepsis had higher mortality: $371 / 905$ (41\%) vs $860 / 2537$ (34\%) HR1.34, 95\%Cl 1.18 to1.52, $p<0.01$; increased costs: ICU: $47,206 \pm 55,121$ vs $34,142 \pm 43,175 ; p<0.0001$; Hospital: 73,516 \pm 61,100 vs $64,676 \pm 56,293 ; p<0.0001$; extended length of stay: ICU: $10.0 \pm 11.9$ vs $7.1 \pm 9.1$ days; $p<0.0001$; Hospital: $22.7 \pm 21.6$ vs 20.4 \pm 19.7 days; $p=0.003$. Patients with sepsis had fewer hospital readmissions: $506 / 904$ (56\%) vs $1597 / 2563$ (63\%); $p=0.0002 .1612$ patients were included in the PSM analysis: 806 with sepsis and 806 without. 90/905 (10\%) patients with sepsis could not be matched due to high illness severity. There were no differences in QoL, late mortality or hospital readmissions. Costs were higher for patients with sepsis: ICU $47,298 \pm 53,730$ vs $38,952 \pm 46,778 ; p=0.009$; Hospital $74,120 \pm 60,750$ vs $65,806 \pm 56,856 ; p=0.005$, and length of stay: ICU: $10.0 \pm 11.9$ vs $8.0 \pm 9.8$ days; $p<0.0001$; Hospital: $22.8 \pm 21.2$ vs $19.1 \pm 19.0$ days; $p=0.0003$.

Conclusions

Compared to patients without sepsis, patients with sepsis report similar QoL but have increased hospital costs, length of stay and fewer hospital readmissions. Aside from cost, these differences are not significant when patients are matched for severity of illness and other baseline variables, notwithstanding the limitation of our inability to match $10 \%$ of patients in the sepsis cohort.

\section{References}

1. Winters, B.D., Eberlein, M., Leung, J., Needham, D., Pronovost, P., Sevransky, J. Long-term mortality and quality of life in sepsis: a systematic review. Crit Care Med, 2010. 38(5): p. 1276-1283.
2. Elliott, D., et al., Exploring the scope of post-intensive care syndrome therapy and care: engagement of non-critical care providers and survivors in a second stakeholders meeting. Crit Care Med, 2014. 42(12): p. 2518-2526

P2

Extracellular histones induce erythrocyte fragility and anaemia and identification of compounds that prevent this process Farzaneh Kordbacheh', Connor H. O'Meara', Lucy A. Coupland', Patrick M. Lelliott ${ }^{2}$, Chris R. Parish ${ }^{1}$

${ }^{1}$ Cancer and Vascular Biology Group, ACRF Department of Cancer Biology and Therapeutics, The John Curtin School of Medical Research, Australian National University, Canberra, Australia; ${ }^{2}$ Department of Immunology and Infectious Disease, John Curtin School of Medical Research, Australian National University, Canberra, ACT, 2601, Australia

Correspondence: Farzaneh Kordbacheh

(farzaneh.kordbacheh@anu.edu.au)

Intensive Care Medicine Experimental 2017, 5(Suppl 1):P2

\section{Background}

Sepsis is a life-threatening systemic inflammatory response to infection or trauma associated with, and mediated by, the activation of a number of host defence mechanisms. Neutrophils play an essential role in sepsis by releasing neutrophil extracellular traps (NETs) which exhibit very effective anti-microbial activity. Uncontrolled formation of NETs can, however, become pathogenic particularly via their associated histones that can be cytotoxic in the vasculature causing endothelial cell death, systemic vascular obstruction and multiple organ failure. They can also initiate coagulation by both activating platelets and damaging erythrocytes such that they become pro-thrombotic. Recently we discovered an additional effect of histones on erythrocyte, which is to enhance their fragility when they are subjected to sheer stress associated with blood flow through the vascular system and spleen in particular. In parallel studies our laboratory has synthesised a family of small polyanions (SPAs) which have been screened for their ability to neutralise the pathogenic effects of histones and NETs.

Methods

We have used a novel mechanically-induced sheer stress method as well as an in vitro spleen filtration model to investigate the effects of mammalian histones \pm SPAs, over a wide concentration range, on RBC fragility under both isotonic and hypotonic conditions. The in vitro findings were mirrored in vivo with the injection of histones and SPAs in mice.

Results

Our results revealed that free histones bind to RBCs and render RBCs susceptible to lysis by shear stress in a dose-dependent manner both in vitro and in vivo models as well as being retained by the spleen. Some SPAs have been identified that can totally prevent the ability of histones to induce RBC fragility in vitro and anaemia in vivo, with the compounds also preventing the rapid and acute histone-induced thrombocytopenia. Remarkably we have also discovered that the active SPAs can rapidly rescue histone damaged enythrocytes, the fragility of the restored RBCs returning to normal levels within minutes, even following prolonged exposure to histones.

Conclusion

Based on our in vitro and in vivo data we propose that erythrocytes can capture histones in the circulation and transport them to the spleen for disposal where overload of this disposal system could

\section{Springer Open}

C) The Author(s). 2017 Open Access This article is distributed under the terms of the Creative Commons Attribution 4.0 International License (http://creativecommons.org/licenses/by/4.0/), which permits unrestricted use, distribution, and reproduction in any medium, provided you give appropriate credit to the original author(s) and the source, provide a link to the Creative Commons license, and indicate if changes were made. 
cause the unexplained anaemia associated with sepsis, cancer and other pathological conditions and our SPAs can both prevent and reverse these effects.

\section{P3}

Innate immune receptor gene polymorphisms and its association with the risk of neonatal sepsis

Snehal L Martin', Saumil Desai ${ }^{2}$, Roshan B Colah', Kanjaksha Ghosh', Ruchi Nanavati ${ }^{2}$, Malay B Mukherjee ${ }^{1}$

${ }^{1}$ Department of Haematogenetics, National Institute of Immunohaematology (ICMR), KEM Hospital campus, Parel, Mumbai, India; ${ }^{2}$ Department of Neonatology, KEM Hospital, Parel, Mumbai, India Correspondence: Snehal Martin (snehalmartin88@gmail.com) Intensive Care Medicine Experimental 2017, 5(Suppl 1):P3

\section{Background}

Neonatal sepsis is one of the major causes of neonatal death in developing countries. Identifying single nucleotide polymorphisms (SNPs) in the genes involved in sepsis may help to understand the pathophysiology of neonatal sepsis. The aim of the present study to look for association between the sepsis in neonates and SNPs in different immune receptor genes involved in host responses to bacterial infection.

\section{Methods}

The study involved 151 preterm neonates with signs and symptoms of clinical sepsis and had a blood culture that was positive for a bacterial or fungal pathogen, 136 preterm neonates with signs and symptoms of sepsis along with a positive septic screen but negative blood culture and 146 healthy neonates born between June 2013 to June 2016. A total of 9 SNPs in 6 innate immune related genes were genotyped using PCR- RFLP and Real time SNP genotyping technique.

Results

Birth weight, gestational age, duration of rupture of membranes, respiratory distress, apnea, abdominal distension, sclerema and anemia were observed in sepsis cases. Incidence of early onset sepsis (EOS) in the blood culture positive neonates was significantly higher as compared to late onset sepsis $(p=0.01)$. Infections caused due to Gram negative organisms (67.92\%) were more common than Gram positive (18.86\%) and fungal infections (13.20\%). An overall mortality rate among the sepsis cases was found to be $26.82 \%$, however, a higher prevalence of mortality (27.94\%) was observed in clinical sepsis as compared to in the proven sepsis $(23.17 \%)$. Although polymorphisms in innate immune related genes did not show significant association with overall risk of developing sepsis, nevertheless, genotype CT of rs5744168 (TLR5 gene) was associated with a significantly reduced risk of developing sepsis $(p=0.02)$. Genotypes TT of rs2569190 (CD14 gene) and CT of rs5744168 (TLR5 gene) were significantly associated with a reduced risk of developing sepsis in the neonates with bacteriologically confirmed sepsis $(p=0.02)$ and clinical sepsis $(p=0.03)$ respectively. Among the patients with bacteriologically confirmed sepsis, genotype CC of rs3804100 (TLR2 gene) was associated with a significantly increased risk of developing severe sepsis ( $p=0.02$ ) while same genotype was found to be reduced the risk of developing sepsis $(p=0.04)$ in the neonates with clinical sepsis.

\section{Conclusions}

This study demonstrates that polymorphisms in the innate immune related genes seems to play a role in sepsis in preterm neonates by influencing susceptibility and the severity of the disease.

\section{Acknowledgement}

We would like to thank Indian council of Medical research (ICMR) for providing fellowship to Ms. Martin S.

\section{P4}

Association of Red cell Distribution Width with sepsis and sepsis related mortality in newborns

Snehal L Martin', Saumil Desai , Roshan B Colah', Kanjaksha Ghosh, Ruchi Nanavati ${ }^{2}$, Malay B Mukherjee ${ }^{1}$

${ }^{1}$ Department of Haematogenetics, National Institute of Immunohaematology (ICMR), KEM Hospital campus, Parel, Mumbai, India; ${ }^{2}$ Department of Neonatology, KEM Hospital, Parel, Mumbai, India Correspondence: Snehal Martin (snehalmartin88@gmail.com) Intensive Care Medicine Experimental 2017, 5(Suppl 1):P4

\section{Background}

Red Cell Distribution Width (RDW) is derived from the routine Complete Blood Count and provides a simple means to stratify patients by severity of illness to facilitate focused interventions without any additional costs. The rise in RDW among neonatal sepsis cases may therefore reflect the degree of the underlying inflammatory state and provide useful prognostic information about the risk of mortality. The present study was undertaken to look for association of Red cell distribution width (RDW) with neonatal sepsis and sepsis related mortality.

\section{Methods}

We performed a prospective observational study on 251 sepsis neonates along with 284 controls. RDW along with other RBC (Red blood cell) indices was performed on an automated cell counter (Sysmex, Japan). We categorized RDW at admission into tertiles of $\leq 16.9 \%, 17$ $20 \%$ and $\geq 20.1 \%$. The cumulative survival rate across the RDW levels during the neonatal period ( $\leq 30$ days) was assessed using the Kaplan-Meier curve and the log rank test.

Results

Of the RBC indices studied, the median (IQR) RDW levels were significantly higher among the neonatal sepsis cases (19.90\%) as compared to the controls $(18.90 \%)(p=0.001)$. The rate of mortality due to sepsis was $28.28 \%$ and RDW was significantly higher amongst the non-survivors (median: 20.30, IQR: 18.3-23.5) than the survivors (median: 19.80, IQR: 17.5-22) ( $p=0.003)$. Kaplan-Meier curve with log rank test showed that increased RDW tertiles are significantly associated with an increased mortality $(p=0.03)$ during the 28-day neonatal period.

Conclusions

The present study suggests that increased RDW is associated with neonatal sepsis and could be an independent outcome predictor for mortality associated with neonatal sepsis. The results of this study might help in the effective management of sepsis in neonates.

\section{Acknowledgement}

We would like to thank Indian council of Medical research (ICMR) for providing fellowship to Ms. Martin S.

P5 Effects of symbiotics on systemic inflammation in animal model of pediatric sepsis

Pricila Romão Marcondes Ávila, Monique Michels, Cleonice Maria Michelon, Maria Vitória Meller, Rafaela Bilésimo, Mariane Abatti, Felipe Dal Pizzol Universidade do Extremo Sul Catarinense- UNESC, Criciúma/SC, Brazil

Correspondence: Pricila Romão Marcondes Avila

(pricilaavila@hotmail.com)

Intensive Care Medicine Experimental 2017, 5(Suppl 1):P5

\section{Background}

Sepsis is a very common clinical syndrome, also affecting pediatric population. The main characteristic of this condition is the imbalance of inflammatory response. A relation between immune system and microbiome has been proposed. This study aimed to investigate the effects of symbiotics on systemic inflammation induced in animal models of pediatric sepsis.

\section{Methods}

Animals were supplemented for 15 consecutive days with prebiotic fructo-oligosaccharides (FOS), probiotics (bifidobacterium bifidum, lactobacillus rhamnosus, lactobacillus casei, lactobacillus acidophilus) or symbiotics. On the 15th day, when the animals were 22 days old, endotoxemia was induced by the administration of lipopolysaccharide from Escherichia coli (LPS). Afterwards, oxidative damage was evaluated by measuring TBARS, carbonyl, and systemic inflammation by myeloperoxidase activity (MPO) and cytokines (IL-1, IL- 6 and TNFa) on the brain, kidney, lung (Fig. 1), and gut (Fig. 2). Next, the role of intestinal microbiota through fecal microbiota transplant (FMT) was evaluated. The fecal donor animals received previous supplementation of two probiotic strains (lactobacillus rhamnosus and lactobacillus casei) for 15 consecutive days. The same parameters of oxidative damages and systemic inflammation already mentioned were evaluated on the brain, kidney, lung (Fig. 3), and gut (Fig. 4). 


\section{Results}

In general, results showed that FOS and probiotics treatment was protective against LPS. In addition, strains had different behaviors on each tissue and in each marker, but their protective effect was more pronounced in the gut tissue. When it was evaluated the role of the intestinal microbiota through FMT, it was observed that FMT was protective, independently of previous administration of probiotics to fecal donor groups.

Conclusion

In conclusion, symbiotics had protective effect upon endotoxemia that was independent of change in intestinal microbiota. However FMT may offer an additional immunomodulatory benefit that was independent on symbiotic modulation.

Keywords: Pediatric sepsis. Probiotics. Symbiotic. Fecal microbiota transplant.
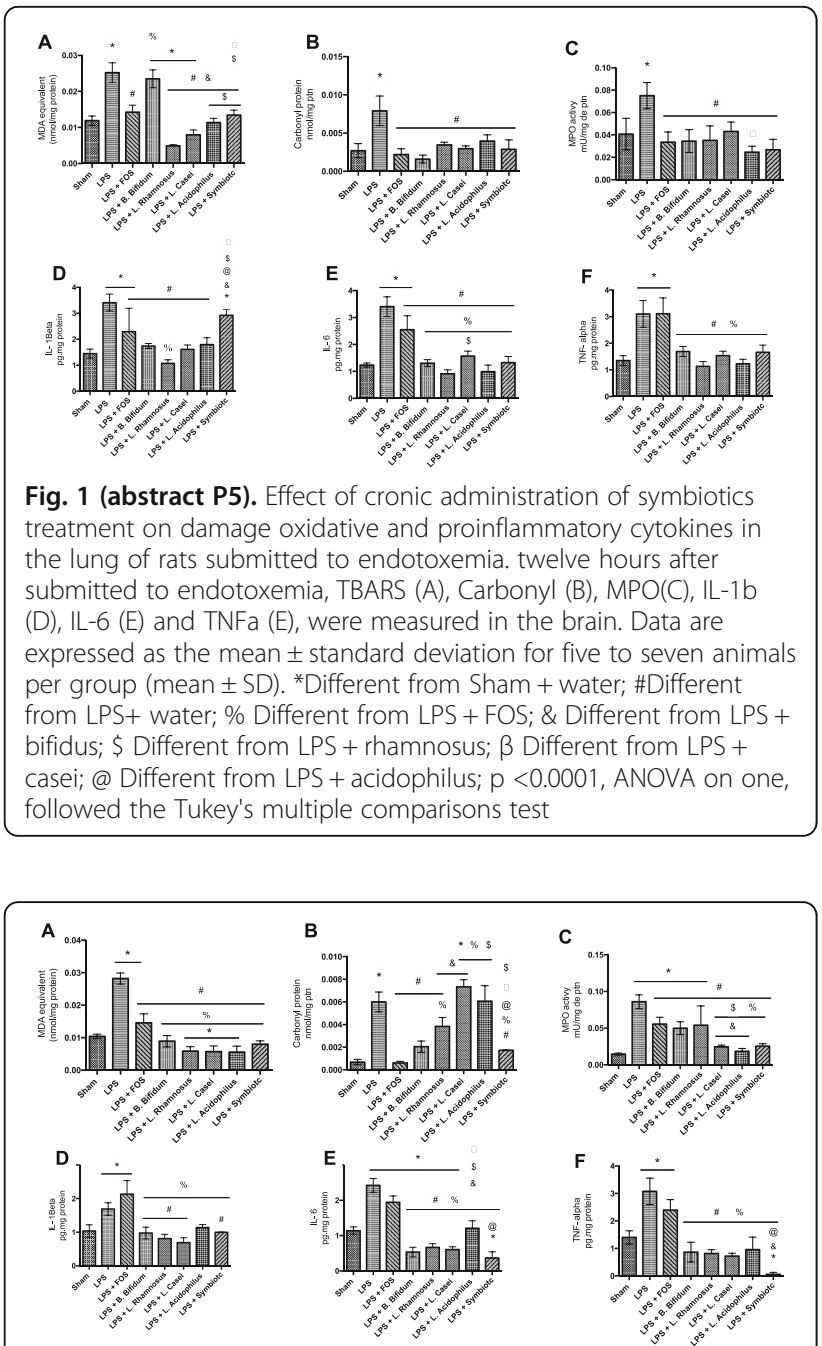

Fig. 2 (abstract P5). Effect of cronic administration of symbiotics treatment on damage oxidative and proinflammatory cytokines in the gut of rats submitted to endotoxemia. twelve hours after submitted to endotoxemia, TBARS (A), Carbonyl (B), MPO(C), IL-1b (D), IL-6 (E) and TNFa (E), were measured in the brain. Data are expressed as the mean \pm standard deviation for five to seven animals per group (mean $\pm \mathrm{SD})$. *Different from Sham + water; \#Different from LPS + water; \% Different from LPS + FOS; \& Different from LPS + bifidus; $\$$ Different from LPS + rhamnosus; $\beta$ Different from LPS + casei; @ Different from LPS + acidophilus; $p<0.0001$, ANOVA on one, followed the Tukey's multiple comparisons test

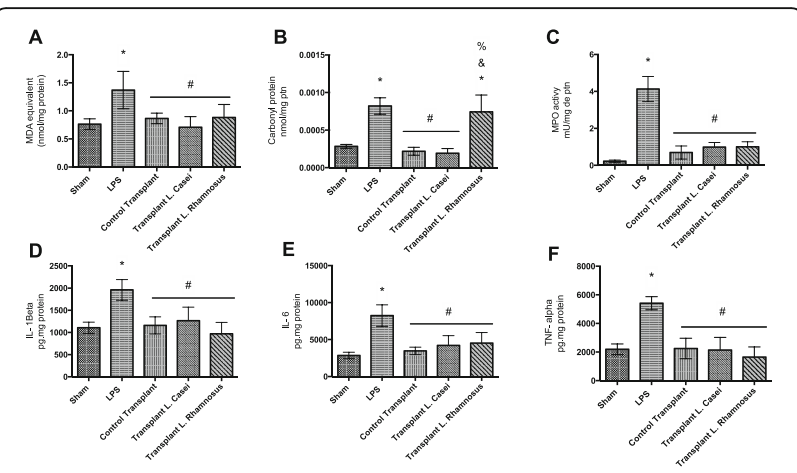

Fig. 3 (abstract P5). Effect of fecal microbiota transplant treatment on damage oxidative and proinflammatory cytokines in the lung of rats submitted to endotoxemia, TBARS (A), Carbonyl (B), MPO(C), IL-1b (D), IL-6 (E) and TNFa (E), were measured. Data are expressed as the mean \pm standard deviation for five to seven animals per group (mean \pm SD). *Different from Sham, \#Different from LPS, \& Different from control transplant, \% Different from transplant I casei; $p<0.0001$

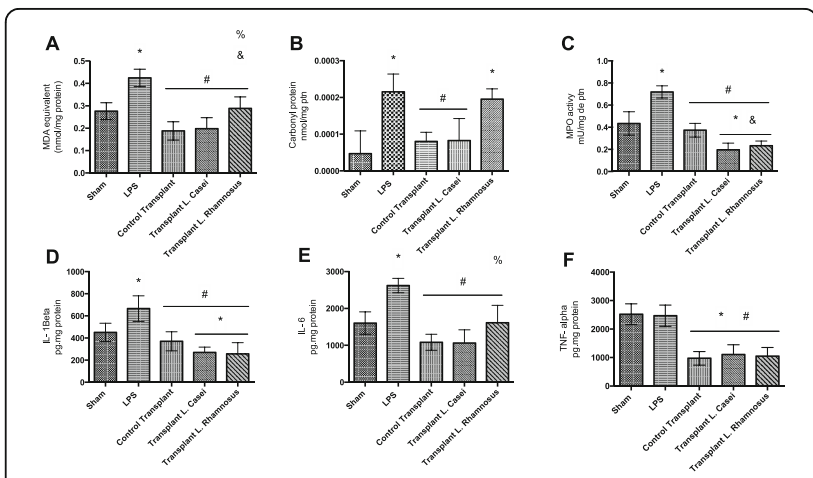

Fig. 4 (abstract P5). Effect of fecal microbiota transplant treatment on damage oxidative and proinflammatory cytokines in the gut of rats submitted to endotoxemia, TBARS (A), Carbonyl (B), MPO(C), IL-1b (D), IL-6 (E) and TNFa (E), were measured. Data are expressed as the mean \pm standard deviation for five to seven animals per group (mean \pm SD). *Different from Sham, \#Different from LPS, \& Different from control transplant, \% Different from transplant I casei; $p<0.0001$

P6

Der $\mathrm{p} 2$ from Dermatophagoides pteronissinus potentiates the endotoxic activity of lipopolysaccharide from Escherichia coli Isabella Prokhorenko', Anastasia Morozova', Dmitry Kabanov', Sergey Grachev ${ }^{1,2}$

${ }^{1}$ FSBSI Institute of Basic Biological Problems RAS, Pushchino, Moscow Region, Russia; ${ }^{2}$ SIEI HE I.M. Sechenov's First Moscow State Medical University of Russian's Ministry of Healthcare, Moscow, Russia

Correspondence: Dmitry Kabanov (kabanovd1@rambler.ru) Intensive Care Medicine Experimental 2017, 5(Suppl 1):P6

\section{Background}

CD14, Toll-like receptor 4 (TLR4) and TLR4-associated adaptor protein MD-2 are essential receptors involved in lipopolysaccharide (LPS) recognition leading to the synthesis and releasing of pro-inflammatory cytokines among them TNF-a playing a fundamental role [1]. It is well known that p38 MAP kinase pathway is involved in TLR4dependent TNF- $a$ production triggered by LPS·MD-2 complexes [2]. Recently, it has been shown that the main component from house dust mite Dermatophagoides pteronissinus extract (DpE) - Der $\mathrm{p} 2$ mimics to MD-2 and acts as LPS.MD-2 complex inducing synthesis of pro-inflammatory cytokines [3, 4]. However, LPS.Der p 2 induced 
intracellular signaling mechanism is not yet known. In this connection the effect of p38 MAP kinase inhibition on TNF-a production induced by LPS or DpE as well as by their combined action has been elucidated in whole blood model ex vivo. It is necessary to note that an inflammatory mechanism in allergic patients with suspected sepsis has not also fully explained.

Methods

Whole blood from healthy volunteers was collected into heparinized tubes (5\%), diluted with RPMI-1640 medium (1:5 v/v), and transferred into CellStar 24-well tissue culture plates. Blood samples were pre-incubated with $10 \mu \mathrm{M}$ of p38 MAP kinase inhibitor SB203580 for $15 \mathrm{~min}$ at $37{ }^{\circ} \mathrm{C}$ in a highly humidified atmosphere containing $5 \% \mathrm{CO} 2$. Then whole blood was stimulated by LPS Escherichia coli O55:B5 (40 ng/ml), DpE (5 $000 \mathrm{PNU} / \mathrm{ml})$ or in their combination during $6 \mathrm{~h}$. When incubation period had been finished, the cell culture plates were centrifuged for $10 \mathrm{~min}$ at $15^{\circ} \mathrm{C}$ and $1000 \mathrm{rpm}$. The supernatants were stored at $-20 \mathrm{C}$ until pending analysis. Concentrations of TNF- $a$ in the supernatants were measured by ELISA Kit according to the manufacturer's instructions. Statistical significance was determined by Mann-Whitney U-test.

Results

The incubation of whole blood with LPS E. coli caused cell activation as determined by TNF-a production, whereas combined applying of LPS E. coli and DpE further increased synthesis of TNF-a (Fig. 5). Very low amounts of TNF-a were detected in the response to DpE alone. Without LPS E. coli or DpE the TNF-a production was almost undetectable. Pre-incubation of whole blood with SB203580 followed by LPS and DpE, either alone or together, resulted in $43 \%$ and $48 \%$ inhibition of LPS-induced TNF-a production as compared to LPS $E$. coli or LPS plus DpE, respectively. DpE triggered TNF-a production was inhibited by SB203580 completely (Fig. 5). These results indicated that the effect of LPS E. coli or DpE on the induction of TNF-a synthesis at least in part depends on p38 MAPK activation. It is necessary to note that there were some variations in TNF-a production triggered by $\mathrm{DpE}$ or DpE and LPS E. coli together. These variations may be related to the use of primary whole blood cells rather than cell lines, with different donors having different sensitivities to the applying drugs.

\section{Conclusions}

In this study we have found that the synthesis of TNF-a was amplified during combined action of LPS E. coli and DpE. The similar suppressive effect of p38 MAP kinase inhibition on LPS- as well as on LPS and DpE-induced synthesis of TNF-a has been revealed. We have supposed that at least two signaling pathways (NF-kB and AP-1) may be involved in the observed TNF-a production $[5,6]$. It seems likely that the DpE leading to TNF-a production does not involve any additional intracellular signaling pathways but probably its effect is realized on the cell surface receptor level $[3,4]$. Our study may result in a better understanding of intracellular signaling mechanisms triggered by combined action of LPS E. coli and Der $\mathrm{p} 2$ from Dermatophagoides pteronissinus extract.

\section{References}

1. Christiansen D, Brekke O, Stenvik J, et al: Differential effect of inhibiting MD-2 and CD14 on LPS- versus whole E. coli bacteria-induced cytokine responses in human blood. Adv Exp Med Biol 2012, 946: 237-251.

2. Fehr S, Unger A, Schaeffeler $E$, et al: Impact of p38 MAP kinase inhibitors on LPS-induced release of TNF-a in whole blood and primary cells from different species. Cell Physiol Biochem 2015, 36: 2237-2249.

3. Park B, Lee $\mathrm{N}$, Kim $\mathrm{M}$, et al: Interaction of Der $\mathrm{p} 2$ with Toll-like receptor 4 and its effect on cytokine secretion. Biomed Sci Lett 2015, 21: 152-159.

4. Trompette A, Divanovic S, Visintin A, et al: Allergenicity resulting from functional mimicry of a Toll-like receptor complex protein. Nature 2009. 457: 585-588.

5. Takashiba S, Shapira L, Amar S, Van Dyke T: Cloning and characterization of human TNF alpha promoter region. Gene 1993, 131: 307-308.

6. Karin M: The regulation of AP-1 activity by mitogen-activated protein kinases. J Biol Chem 1995, 270: 16483-16486.

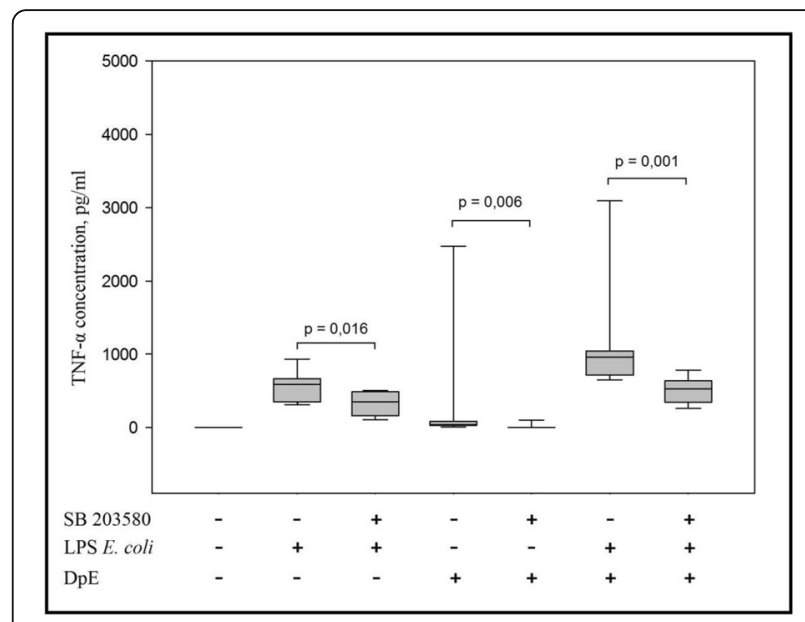

Fig. 5 (abstract P6). See text for description

P7

Chemiluminescent analysis of the neutrophil function: from bench to bedside

Igor V. Obraztsov ${ }^{1,3}$, Marina A. Sukhina', Vadim N. Sinyutin², Sergey I. Achkasov', Oleg I. Sushkov', Anton L. Safin ${ }^{1}$

${ }^{1}$ A. N. Ryzhikh State Scientific Centre for Coloproctology, Moscow, Russia; ${ }^{2}$ M. V. Lomonosov Moscow State University, Moscow, Russia; ${ }^{3} \mathrm{D}$. Rogachev National Scientific and Clinical Centre for Pediatric Haematology, Oncology and Immunology, Moscow, Russia

Correspondence: Igor Obraztsov (igor_obraztsov@bk.ru) Intensive Care Medicine Experimental 2017, 5(Suppl 1):P7

\section{Background}

Neutrophils play a crucial role in antimicrobial resistance; evaluation of reactive oxygen species (ROS) production by the neutrophils could provide valuable clinical data on neutrophils' microbicidal capacity. Our aim was to develop a standardized methodology for the neutrophil's oxidative output assessment and to implement it in a clinical practice for management of surgical patients.

Methods

Oxidative metabolism was evaluated in the whole blood by means of luminol-enhaced chemiluminescence $(\mathrm{CL})$ induced by priming with $50 \mathrm{ng} / \mathrm{ml}$ 4-phorbol-12-myristate-13-acetate (PMA) prior to $10 \mu \mathrm{M} \mathrm{N}$-formyl-methionyl-leucyl-phenylalanine (fMLP) stimulation. We defined an experiment design in order to obtain a signal of high intensity and reproducible pattern. We assessed the time-dependent neutrophils' phenotypic shift by means of CD64, CD16, CD62L, CD11b, CD15 and CD33 expression evaluation after PMA stimulation.

Results

We revealed changes of CD62L $(p<0,05)$, CD11b $(p<0,01)$ and CD15 $(p<0,05)$ expression as well as CD11b/CD15(high) and CD11b/ CD15(bright) subsets formation $(p=0,001)$ under PMA priming of the neutrophils. Surface markers expression under PMA stimulation has various impacts on oxidative output kinetics. PMA priming of the neutrophils also leads to a dramatic increase of $\mathrm{AMLP}$-induced ROS production $(p<0,001)$ that is accompanied by reduction of relative intracellular ROS production $(p<0,001)$. We built reference $C L$ parameters in a control group of 95 healthy individuals. Investigation of 17 patients with severe burns in dynamics revealed association between intracellular CL insufficiency and development of severe septic complications. We built a fitting linear-logarithmic function to create an unbiased approach to intracellular $C L$ quantification and substantiated clinically relevant kinetic parameters (Fig. 6). We used prognostic coefficient $C=12 / 13$ to perform a precise quantification of intracellular oxidative output in 21 colorectal cancer patients after surgery in 
dynamics. CD16, CD64 as well as CD11b expression by the CD11b/ CD15(bright) subset drive $C$ to the higher levels $(p<0,05)$, while CD62L and CD11b expression by the CD11b/CD15(high) subset tend to low $C(p<0,05)$. Assessment of colorectal cancer patients on the first four days after surgery has shown an increase of $C(p<0,05)$ as well as CD64+ neutrophils $(p<0,01)$ and procalcitonin $(p<0,01)$ levels in patients with longer hospitalization duration due to subsequent inflammatory complications.

Conclusion

Thus kinetic $\mathrm{CL}$ assay being high-sensitive, cost-effective, easy-tostandardize and easy-to-perform, is promising for use in a clinical routine of a surgical hospital.

\section{Acknowledgement}

Authors express their deepest gratitude to prof. Yu. A. Vladimirov and all the stuff of the department for medical biophysics at M. V. Lomonosov Moscow State University for the encouragement, advice and help.

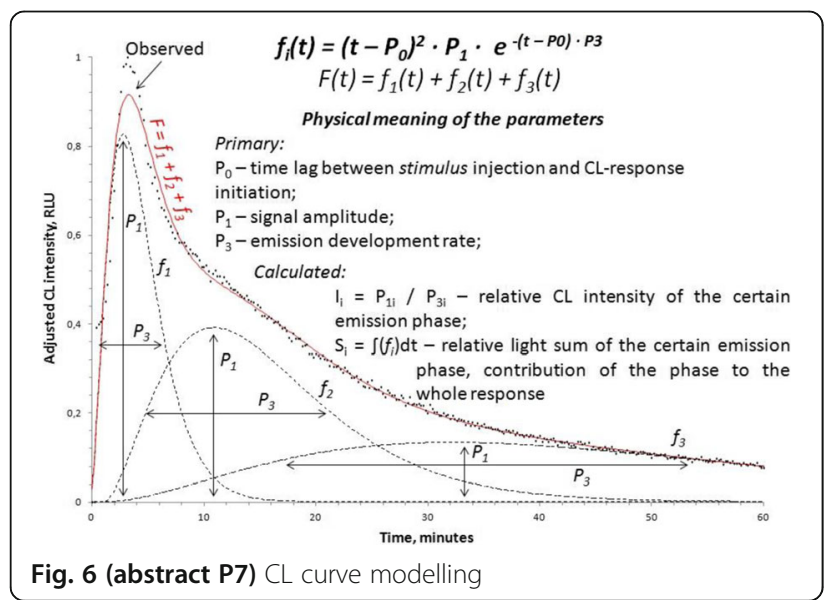

\section{P8}

\section{Withdrawn}

P9

Preventive diagnosis of sepsis in newborns with respiratory diseases on mechanical ventilation

Marina Puchtinskaya, Viadimir Estrin

Department of Anesthesiology and Critical Care Medicine,

Rostov-on-Don State Medical University, Rostov-on-Don, Russia

Correspondence: Marina Puchtinskaya (puhmar@mail.ru)

Intensive Care Medicine Experimental 2017, 5(Suppl 1):P9

\section{Background}

The decision on the confirmation of the diagnosis of sepsis remains a challenge of neonatology[1,2]. The purpose of this study was to improve the efficiency of preventive diagnosis of sepsis in newborns with respiratory diseases on mechanical ventilation.

Methods

The presentation of the monitored, randomized clinical trials was approved by the local committee on ethical issues and bioethics of the Research Institute of Obstetrics and Pediatrics, Rostov-on-Don, Russia (protocol №8/1 of 26.11.2008 and №20/1 of 14.01.2009). Patients were included in the study with the written permission of their parents or other legal representatives. The text of the authorization and the research protocol are formulated in accordance with the Declaration of Helsinki, Federal Law N323-F of the Zot on November 21, 2011 "On the fundamentals of protecting the health of citizens in the Russian Federation"; the basis of the legislation of the Russian Federation "On the protection of public health, the rules for clinical practice in the Russian Federation" (Order of the Ministry of Health of the Russian Federation N266 of 19.07.03, order of Roszdravnadzor N2325-Pr/06 of 17.10.06). 200 full-term newborns with respiratory pathology, admitted to the neonatal intensive care unit on a ventilator in 1 day of life, without clinical signs of bacterial infection on admission, 3-5, 20 days was determined by ELISA (Victor Multilabel Coulter-21420, Finland) plasma concentration of IL-1ß, IL-6, IL-8, TNF-a, G-CSF, s-Fas, FGF, NO; method of immunophenotyping (Beckman Coulter Epics XL-II, USA) CD3 + CD19-, CD3-CD19+, CD3 + CD4+, CD3 + CD8+, CD69+, CD71+, CD95+, HLA-DR+, CD34+, CD14+, CD3-CD56+; lymphocytes with the expression of AnnexinV-FITC + PI- and AnnexinV-FITC + PI+. Retrospectively the patients were divided into two groups: with sepsis and without sepsis. Cluster analysis formed the base of the immunological data of preventive diagnosis of sepsis, the assessment of the value of the correlation coefficient by the method of "decision Trees" was performed in the statistical environment "R".

Results

Of the 200 patients develop sepsis confirmed at 45 . Statistical cluster analysis of a set of immunological criteria of diagnosis of sepsis at admission to ICU confirmed the presence of two clusters (the presence or absence of sepsis: these two components explain $60.81 \%$ of the point variability). The method of "decision Trees" formulated the diagnostic rule for preventive diagnosis of sepsis: if $\mathrm{CD} 95 \geq 16.8 \% \mathrm{NO} \leq 9.6 \mathrm{mkmol} / \mathrm{l}$ or $\mathrm{CD} 95 \leq 16.8 \%$ and $\mathrm{CD} 34 \leq 0.2 \%$ and $\mathrm{CD} 69 \geq 4.12 \%$ or CD95 $\leq 16.8 \%$ and $\mathrm{CD} 34 \leq 0.2 \%$ CD69 $\leq 4.12 \%$ and lymphocytes with the expression of AnnexinV-FITC $+\mathrm{PI}-\geq 12.3$ per cent - is the development of sepsis. Diagnostic accuracy $95.41 \%$; sensitivity $97.06 \%$; specificity $94.67 \%$; false-positive diagnoses, the share of $5.33 \%$; the proportion of false-negative diagnoses of $2.94 \%$, the accuracy of a positive result $89.19 \%$; the accuracy of a negative result up $98.61 \%$.

Conclusions

Determination of the relative content of lymphocyte expression of CD95+, CD69+, AnnexinV-FITC + Pl-; stem cells CD34+ and plasma NO concentration to diagnose sepsis in newborns with respiratory pathology on a ventilator at admission to the NICU. A significant role in the development of sepsis belongs to prevalence of process of alteration of immunocompetent cells on the proliferation and endogenous synthesis of nitric oxide.

\section{References}

1. Puchtinskaya M., Estrin V. Prevention of sepsis by correcting apoptosis// Critical Care. - 2013. - Vol. 17, Suppl 2. - P. 8.

2. Puchtinskaya M. Effect of inhaled nitric oxide on apoptosis of lymphocytes in newborns in critical states//Critical Care. - 2014. - Vol. 18, Suppl 1. - P. 288.

P10

The mortality attributable to sepsis in adult patients in intensive care Marinelle Schout ${ }^{1,2,3}$, Naomi Hammond ${ }^{2,3,4}$, Frances Bass ${ }^{2,3}$, Anthony Delaney $^{2,3,4}$, Simon Finfer 2,3,4 $^{2,4}$

${ }^{1}$ Radboud University, Nijmegen, The Netherlands; ${ }^{2}$ Intensive Care Unit, Royal North Shore Hospital, Sydney, Australia; ${ }^{3}$ University of Sydney, Sydney, Australia; ${ }^{4}$ The George Institute for Global Health, Sydney, Australia Correspondence: Marinelle Schout (marinelleschout@gmail.com) Intensive Care Medicine Experimental 2017, 5(Suppl 1):P10 


\section{Background}

Chronic comorbid conditions are present in the majority of sepsis patients and these patients have a significantly worse outcome than patients without comorbidities [1,2]. It is thought that sepsis occurs as a terminal event in a proportion of sepsis patients dying from a disease process other than sepsis $[1,3]$

Methods

We conducted an inception cohort study including all patients admitted to the ICU at a tertiary hospital in Sydney, Australia between October 1st 2016 and December 31st 2016. In all patients diagnosed with sepsis using clinical criteria[4] we used an adjudication system where clinicians estimated the pre-sepsis life expectancy of patients who died in-hospital within 60 days. Patients who had an estimated life expectancy of less than 90 days were adjudicated to have died with sepsis rather than from sepsis.

Results

From 864 patients admitted, $146(16.9 \%)$ patients were prospectively diagnosed with sepsis and included (Fig. 7). Comorbidities were present in 117/146 (80.1\%) of patients (Table 1). 39/146 (26.7\%) died in-hospital within 60 days. 30/39 (76.9\%) of deceased patients were adjudicated not to be in a terminal phase of an underlying disease process before onset of sepsis. These deaths were therefore adjudicated to be directly attributable to sepsis.

Conclusions

Despite the presence of comorbidities in the majority of patients, most patients who died were not in a terminal stage of an underlying disease process when sepsis occurred. Therefore, sepsis did result in an appreciable loss of life years. Further research in this area is warranted.

\section{References}

1. Angus DC, Linde-Zwirble WT, Lidicker J, Clermont G, Carcillo J, Pinsky MR. Epidemiology of severe sepsis in the United States: analysis of incidence, outcome, and associated costs of care. Crit Care Med. 2001:29(7):1303-1310

2. Kaukonen KM, Bailey M, Suzuki S, Pilcher D, Bellomo R. Mortality related to severe sepsis and septic shock among critically ill patients in Australia and New Zealand, 2000-2012. JAMA. 2014;311(13):1308-1316

3. Daviaud F, Grimaldi D, Dechartres A, et al. Timing and causes of death in septic shock. Ann Intensive Care. 2015;5(1):16.

4. Finfer S, Bellomo R, Lipman J, French C, Dobb G, Myburgh J. Adult-population incidence of severe sepsis in Australian and New Zealand intensive care units. Intensive Care Med. 2004;30(4):589-596.

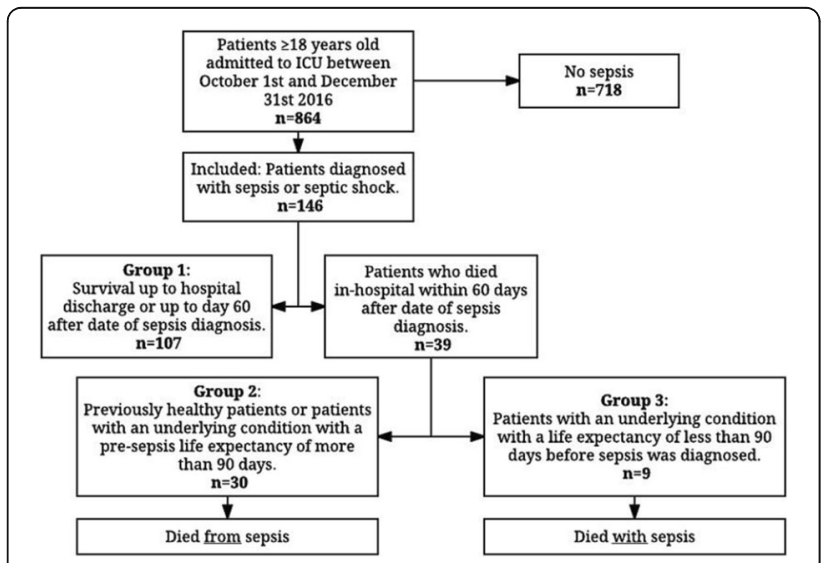

Fig. 7 (abstract P10). Inclusion and adjudication process
Table 1 (abstract P10). Patient characteristics of all included patients and per adjudication group

\begin{tabular}{|c|c|c|c|c|c|}
\hline & $\begin{array}{l}\text { All } \\
\text { patients } \\
\mathrm{N}=146\end{array}$ & $\begin{array}{l}\text { Group } \\
1^{*} \\
N=107\end{array}$ & $\begin{array}{l}\text { Group } \\
2^{* *} \\
\mathrm{~N}=30\end{array}$ & $\begin{array}{l}\text { Group } \\
3^{* * *} \\
\mathrm{~N}=9\end{array}$ & $\begin{array}{l}\mathrm{p} \text { - } \\
\text { value } \\
* * * *\end{array}$ \\
\hline Age, mean (SD) & $\begin{array}{l}64.5 \\
(15.4)\end{array}$ & $\begin{array}{l}64.4 \\
(15.7)\end{array}$ & $\begin{array}{l}65.6 \\
(14.0)\end{array}$ & $\begin{array}{l}62.0 \\
(17.6)\end{array}$ & 0.53 \\
\hline Male sex, $n$ (\%) & $90(61.6)$ & $70(65.4)$ & $13(43.3)$ & $6(66.7)$ & 0.27 \\
\hline \multicolumn{6}{|l|}{ Comorbidities, $n(\%)$} \\
\hline Myocardial infarction & $20(13.7)$ & $16(15.0)$ & $4(13.3)$ & $0(0.0)$ & 0.56 \\
\hline Congestive heart failure & $14(9.6)$ & $10(9.3)$ & $3(10.0)$ & $1(11.1)$ & 0.99 \\
\hline Peripheral vascular disease & $8(5.5)$ & $8(7.5)$ & $0(0.0)$ & $0(0.0)$ & N/A \\
\hline Cerebrovascular disease & $13(8.9)$ & $10(9.3)$ & $3(10.0)$ & $0(0.0)$ & 0.99 \\
\hline Dementia & $7(4.8)$ & $4(3.7)$ & $310.0)$ & $0(0.0)$ & 0.99 \\
\hline Chronic pulmonary disease & $31(21.2)$ & $19(17.8)$ & $11(36.7)$ & $1(11.1)$ & 0.23 \\
\hline Connective tissue disease & $14(9.6)$ & $7(6.5)$ & $5(16.7)$ & $2(22.2)$ & 0.65 \\
\hline Ulcer disease & $6(4.1)$ & $4(3.7)$ & $2(6.7)$ & $0(0.0)$ & 0.99 \\
\hline Mild liver disease & $12(8.2)$ & $8(7.5)$ & $2(6.7)$ & $2(22.2)$ & 0.22 \\
\hline Diabetes & $33(22.6)$ & $21(19.6)$ & $8(26.7)$ & $4(44.4)$ & 0.42 \\
\hline Hemiplegia & $10(6.8)$ & $9(8.4)$ & $1(3.3)$ & $0(0.0)$ & 0.99 \\
\hline Moderate/severe renal disease & $5(3.4)$ & $4(3.7)$ & $0(0.0)$ & $1(11.1)$ & 0.23 \\
\hline $\begin{array}{l}\text { Diabetes with end organ } \\
\text { damage }\end{array}$ & $3(2.1)$ & $3(2.8)$ & $0(0.0)$ & $0(0.0)$ & N/A \\
\hline Any (solid) tumour & $9(6.2)$ & $7(6.5)$ & $2(6.7)$ & $0(0.0)$ & 0.99 \\
\hline Leukaemia & $8(5.5)$ & $5(4.7)$ & $3(10.0)$ & $0(0.0)$ & 0.99 \\
\hline Lymphoma & $5(3.4)$ & $3(2.8)$ & $0(0.0)$ & $2(22.2)$ & 0.05 \\
\hline Moderate/severe liver disease & $5(3.4)$ & $2(1.9)$ & $2(6.7)$ & $1(11.1)$ & 0.56 \\
\hline Metastatic solid tumour & $13(8.9)$ & $7(6.5)$ & $3(10.0)$ & $3(33.3)$ & 0.12 \\
\hline $\begin{array}{l}\text { Comorbidities per patient, } \\
\text { mean (SD) }\end{array}$ & $1.5(1.1)$ & $1.4(1.1)$ & $1.8(0.9)$ & $1.9(1.1)$ & - \\
\hline $\begin{array}{l}\text { Patients with } \geq 1 \text { comorbidity, } n \\
\text { (\%) }\end{array}$ & $117(80.1)$ & $82(76.6)$ & $27(90.0)$ & $8(88.9)$ & - \\
\hline CCS, median (IQR) & $2(1-3)$ & $2(1-3)$ & $2(1-3)$ & $4(2-6)$ & 0.14 \\
\hline None (0), $n(\%)$ & $29(19.9)$ & $25(23.4)$ & $3(10.0)$ & $1(11.1)$ & - \\
\hline Low (1-2), n (\%) & $70(47.9)$ & $53(49.5)$ & $15(50.0)$ & $2(22.2)$ & - \\
\hline Moderate (3-4), n (\%) & $28(19.2)$ & $19(17.8)$ & $7(23.3)$ & $2(22.2)$ & - \\
\hline High ( $\geq 5), n(\%)$ & $19(13.0)$ & $10(9.3)$ & $5(16.7)$ & $4(44.4)$ & - \\
\hline
\end{tabular}

*Patients who survived up to hospital discharge, or up to day 60 after sepsis diagnosis. **Patients who died in-hospital within 60 days after sepsis diagnosis with a pre-sepsis life expectancy of at least 90 days. ***Patients who died in-hospital within 60 days after sepsis diagnosis with a pre-sepsis life expectancy of less than 90 days. ****P-values refer to the difference between Group 2 and Group 3

P11

Automated screening system for sepsis in hospital wards (Serial Patient Screen for Infectious Source, SePSIS)

Rosemary Grant ${ }^{1}$, Natalie Kress², Benjamin Wolpaw², David Carlbom ${ }^{3}$ ${ }^{1}$ Clinical Education, Harborview Medical Center, Seattle, WA, USA;

${ }^{2}$ University of Washington School of Medicine, Seattle, WA, USA;

${ }^{3}$ Pulmonary and Critical Care Medicine, Harborview Medical Center, Seattle, WA, USA

Correspondence: Rosemary Grant (mitchell@uw.edu) Intensive Care Medicine Experimental 2017, 5(Suppl 1):P11

\section{Background}

Early identification and timely intervention are essential in the management of patients with sepsis. Numerous clinical studies have shown that patients developing sepsis on acute care hospital wards often 
experience delays in diagnosis and treatment resulting in increased length of stay, ICU transfer, and higher mortality. At Harborview Medical Center, hospital-acquired sepsis is associated with a $26 \%$ mortality rate, which is $70 \%$ worse than expected and markedly elevated compared just $5 \%$ for sepsis present on admission.

Our sepsis early detection and warning system (Serial Patient Screen for Infectious Source, SePSIS) was implemented in 2012 to address this disparity. SePSIS makes use of vital sign and laboratory data present in the electronic health record (EHR) to generate an automated screening form to query bedside nurses for suspicion of infection. Concern for infection is subsequently communicated to providers to prompt additional diagnostic tests and treatment interventions. The SePSIS warning system is very sensitive (89\%), but not very specific $(51 \%)$, leading to a high number of false positive screens and subsequent alert fatigue.

Using a Rapid Process Improvement (RPI), we assembled a multidisciplinary team of nurses and physicians. With senior physician and process improvement specialist mentorship, we re-designed and implemented a successful overhaul of our current screening system, focusing on improving workflow delays, communication and user satisfaction.

Methods

We identified three areas for improvement: 1) Our low specificity algorithm, 2) Delays in response times to automated alerts, and 3) Lack of closed-loop communication. We simplified the algorithm, improved workflow, created a new "Sepsis Order Set," and trained our teams. Clinical informatics staff helped develop refinements to our algorithm.

We will track several outcomes in admitted patients with a discharge diagnosis of sepsis: Mortality, Length of Stay, ICU Transfer Rate, Time to Completion of 3 Hour Bundle.

Results

In February 2017 we implemented the workflow changes. We will implement our updated algorithm and expect improved specificity.

Ten weeks after implementation, rates of compliance with our order set reached 54\%, markedly higher than previously implemented order sets at our institution. Nurses now complete screening tasks within two hours in $90 \%$ of alerts.

We believe that systematic changes to our EHR-based sepsis screening will improve time to delivery and reduce mortality.

Conclusions

Automated screening for sepsis in acute care hospital wards may be helpful in the early identification and treatment of sepsis.

P12

The incidence of sepsis in an Australian ICU: a prospective clinical diagnosis versus a retrospective database diagnosis Manon Heldens ${ }^{1,2,3}$, Naomi Hammond ${ }^{2,3,4}$, Frances Bass ${ }^{2,3,4}$ Anthony Delaney ${ }^{2,3}$, Simon Finfer $2,3,4$

${ }^{1}$ Radboud University, Nijmegen, The Netherlands; ${ }^{2}$ Intensive Care Unit, Royal North Shore Hospital, Sydney, Australia; ${ }^{3}$ University of Sydney, Sydney, Australia; ${ }^{4}$ The George Institute for Global Health, Sydney, Australia

Correspondence: Manon Heldens (heldensmanon@gmail.com) Intensive Care Medicine Experimental 2017, 5(Suppl 1):P12

\section{Background}

Estimates of the incidence of sepsis are often based on retrospective database or coding studies and the accuracy of these estimates in comparison with prospective cohort studies is not clear. The objective of this study is to compare the incidence of sepsis derived from a prospective inception cohort study (clinically diagnosed sepsis) and that derived from retrospective database (Australian and New Zealand Intensive Care Society Evaluation Centre for Outcome and Resource Evaluation (ANZICS CORE)) coding in ICU patients.

Methods

Design, setting, patients: This study is an inception cohort study including all patients admitted between the 1st of October and the 31st of December 2016 at the ICU of The Royal North Shore Hospital,
St Leonards, Australia. Main outcome measures: Incidence of sepsis and septic shock using a clinical and a database definition.

Results

A total of 864 patients were admitted during the study period (Table 2), of these 146 patients $(16.9 \%)$ met the clinical definition of sepsis, of which 49/146 (33.6\%) met the clinical definition for septic shock. A total of $98 / 864$ patients (11.3\%) met the database definition of sepsis, with 83/98 (84.7\%) diagnosed with septic shock (Figs. 8 and 9). Hospital mortality was higher in patients who met the clinical definition of sepsis $(39 / 146,26.7 \%)$, compared to patients that met the database definition of sepsis (17/ $98,17.3 \%)$.

Conclusions

Our study showed that the incidence of sepsis using the ANZICS CORE database definition is lower and the incidence of septic shock higher when compared with a prospective clinical definition. The database definition also provides a falsely low estimate of sepsis mortality.

\section{Acknowledgement}

Radboud Honours Programme 'Beyond the Frontiers'

Table 2 (abstract P12). Patient characteristics

\begin{tabular}{|c|c|c|c|c|c|c|c|}
\hline \multirow[t]{2}{*}{ Groups $^{a}$} & $\begin{array}{l}\text { All } \\
\text { patients }\end{array}$ & $\begin{array}{l}\text { Clinical } \\
\text { Sepsis }\end{array}$ & $\begin{array}{l}\text { No } \\
\text { clinical } \\
\text { sepsis }\end{array}$ & $\begin{array}{l}\text { Database } \\
\text { sepsis }\end{array}$ & $\begin{array}{l}\text { No } \\
\text { database } \\
\text { sepsis }\end{array}$ & Neither & \multirow[t]{2}{*}{$\begin{array}{l}\mathrm{P} \\
\text { values }^{\mathrm{d}}\end{array}$} \\
\hline & $n=864$ & $n=146$ & $n=718$ & $\mathrm{n}=98$ & $n=766$ & $\begin{array}{l}n= \\
696\end{array}$ & \\
\hline Male, n (\%) & $\begin{array}{l}484 \\
(56.0)\end{array}$ & $\begin{array}{l}86 \\
(58.9)\end{array}$ & $\begin{array}{l}398 \\
(55.2)\end{array}$ & $50(51.0)$ & $434(56.7)$ & $\begin{array}{l}385 \\
(55.3)\end{array}$ & 0.24 \\
\hline $\begin{array}{l}\text { APACHE III } \\
\text { score, mean } \\
(\text { SD) }\end{array}$ & $\begin{array}{l}45.0 \\
(23.5)\end{array}$ & $\begin{array}{l}62.2 \\
(24.7)\end{array}$ & $\begin{array}{l}41.4 \\
(21.7)\end{array}$ & $\begin{array}{l}59.8 \\
(25.6)\end{array}$ & $\begin{array}{l}43.1 \\
(22.5)\end{array}$ & $\begin{array}{l}41.4 \\
(21.7)\end{array}$ & 0.44 \\
\hline $\begin{array}{l}\text { Age in years, } \\
\text { mean (SD) }\end{array}$ & $\begin{array}{l}62.1 \\
(17.6)\end{array}$ & $\begin{array}{l}64.4 \\
(15.4)\end{array}$ & $\begin{array}{l}61.6 \\
(18.0)\end{array}$ & $\begin{array}{l}64.7 \\
(15.6)\end{array}$ & $\begin{array}{l}61.8 \\
(17.8)\end{array}$ & $\begin{array}{l}61.6 \\
(18.2)\end{array}$ & 0.89 \\
\hline $\begin{array}{l}\text { Postoperative } \\
\text { admissions, n } \\
(\%)\end{array}$ & $\begin{array}{l}380 \\
(44.0)\end{array}$ & $\begin{array}{l}19 \\
(13.0)\end{array}$ & $\begin{array}{l}361 \\
(50.3)\end{array}$ & $12(12.2)$ & $368(48.0)$ & $\begin{array}{l}354 \\
(50.9)\end{array}$ & 0.99 \\
\hline $\begin{array}{l}\text { Length of ICU } \\
\text { stay in days, } \\
\text { median (IQR) }\end{array}$ & $2(1-4)$ & $\begin{array}{l}4.5(2- \\
8)\end{array}$ & $2(1-3)$ & $3(2-6.8)$ & $2(1-3)$ & $2(1-3)$ & 0.02 \\
\hline $\begin{array}{l}\text { Died in ICU, n } \\
(\%)\end{array}$ & $64(7.4)$ & $\begin{array}{l}32 \\
(21.9)\end{array}$ & $32(4.5)$ & $16(16.3)$ & $48(6.3)$ & $31(4.5)$ & 0.33 \\
\hline $\begin{array}{l}\text { Died in } \\
\text { hospital, } n(\%)^{c}\end{array}$ & $83(9.6)$ & $\begin{array}{l}39 \\
(26.7)\end{array}$ & $44(6.1)$ & $17(17.3)$ & $66(8.6)$ & $42(6.0)$ & 0.12 \\
\hline $\begin{array}{l}\text { Died within } \\
28 \text { days, } n(\%)^{c}\end{array}$ & $76(8.8)$ & $\begin{array}{l}34 \\
(23.3)\end{array}$ & $42(5.8)$ & $14(14.3)$ & $62(8.1)$ & $41(5.9)$ & 0.10 \\
\hline $\begin{array}{l}\text { Septic shock, n } \\
\text { (\%) }\end{array}$ & & $\begin{array}{l}49 \\
(33.6)\end{array}$ & & $83(84.7)$ & & & $<0.0005$ \\
\hline $\begin{array}{l}\text { Died in ICU, n } \\
(\%)\end{array}$ & & $\begin{array}{l}\bullet 18 \\
(36.7)\end{array}$ & & •12 (14.5) & & & 0.005 \\
\hline $\begin{array}{l}\text { Died in } \\
\text { hospital, n (\%) }\end{array}$ & & $\begin{array}{l}\bullet 18 \\
(36.7)\end{array}$ & & •13 (15.7) & & & 0.01 \\
\hline $\begin{array}{l}\text { Died within } \\
28 \text { days, } n(\%)^{c}\end{array}$ & & $\begin{array}{l}\bullet 16 \\
(32.7)\end{array}$ & & •11 (13.3) & & & 0.013 \\
\hline $\begin{array}{l}\text { No septic } \\
\text { shock, } \mathrm{n}(\%)\end{array}$ & & $\begin{array}{l}97 \\
(66.4)\end{array}$ & & $15(15.3)$ & & & $<0.0005$ \\
\hline
\end{tabular}

${ }^{a}$ Patients admitted more than once were classified as sepsis if they had sepsis during any admission and classified as not having sepsis if they did not have sepsis during any admission admission and classified as not having sepsis if they did not have sepsis during any admission
b To calculate the APACHE III score and length of stay for those who did not have sepsis, data from the first admission is included in this table. For those who did have sepsis, data from the first admission with sepsis is included in this table

C For mortality data all patients were followed until hospital discharge

${ }^{d} \mathrm{P}$ values refer to the difference between the clinical sepsis group and the database sepsis group 


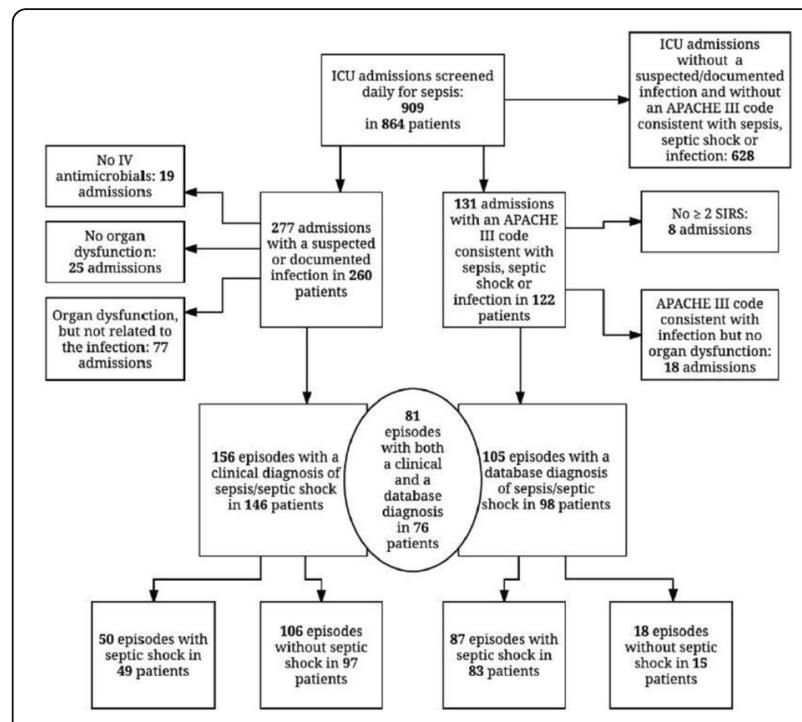

Fig. 8 (abstract P12). Outcome of $909 \mathrm{ICU}$ admissions. The difference between the number of admissions and patients is due to readmissions

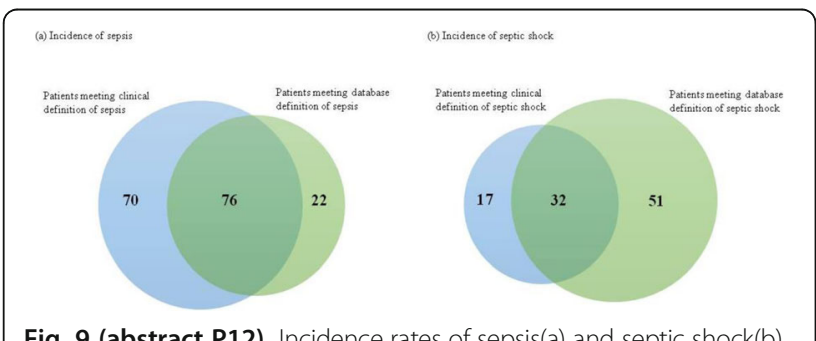

Fig. 9 (abstract P12). Incidence rates of sepsis(a) and septic shock(b)

P13

Antibiotic administration and lactate level measurement timing in septic shock patients admitted in the medical ICU: hospital ward versus emergency department

Veerapong Vattanavanit ${ }^{1}$, Theerapat Buppodom ${ }^{2}$

${ }^{1}$ Department of Internal Medicine, Prince of Songkla University, Hat Yai, Songkhla, Thailand; ${ }^{2}$ Faculty of Medicine, Prince of Songkla University, Hat Yai, Songkhla, Thailand

Correspondence: Theerapat Buppodom (ph.theera13@gmail.com) Intensive Care Medicine Experimental 2017, 5(Suppl 1):P13

\section{Background}

The timing of lactate level measurement and the timing of intravenous antibiotics administration are associated with survival of septic shock patients. Septic shock patients were admitted to medical intensive care unit (MICU) from 2 major sources, hospital ward and emergency department. The differences in management on each sources will affect the lactate and intravenous antibiotics timing.

Methods

The medical data of adult patients with septic shock from hospital wards and emergency department, admitted to the MICU, Songklanagarind hospital, during January 2015 to December 2016, were collected. Timing of antibiotic administration and lactate level measurement from "time zero" (time diagnosis sepsis) were recorded. The association between timing and risk-adjusted 28-day mortality are assessed.

Results

Among 150 septic shock patients admitted to medical ICU, 58.7\% were from hospital ward. The median time (min) from time zero to antibiotic administration time was higher in hospital ward compared with the emergency department (290.5, interquartile range (IQR), 210 to 486 versus 122 , IQR 82 to 212 ; $P<0.10)$. With the same trend of lactate level measurement timing ( $\mathrm{min}$ ) (97, IQR, 42 to 164 versus $95, \mathrm{IQR}, 57$ to 183 ; $P=0.37$ ). A longer timing of antibiotic administration was associated with higher risk-adjusted 28-day mortality (odds ratio 0.99 ; $95 \%$ confidence interval, 0.99 $0.99 ; P<0.05)$.

Conclusions

Septic shock patients from hospital wards had longer timing of antibiotic administration and lactate level measurement.

P14

Antibacterial and antibiofilm activity of silver nanoparticles with reducing agent extracted from Eucalyptus coating on endotracheal tube surface: phase I study in the 'PSU endotracheal tube' innovation project

Bodin Anantravanit ${ }^{1}$, Veerapong Vattanavanit', Chalongrat Daengngam², Supayang Voravuthikunchai ${ }^{3}$

${ }^{1}$ Department of Internal Medicine, Faculty of Medicine, Prince of Songkla University, Hat Yai, Songkhla, Thailand; ${ }^{2}$ Department of Physics, Faculty of Science, Prince of Songkla University, Hat Yai, Songkhla, Thailand; ${ }^{3}$ Department of Microbiology, Faculty of Science, Prince of Songkla University, Hat Yai, Songkhla, Thailand

Correspondence: Bodin Anatravanit (Benfyrere@hotmail.com) Intensive Care Medicine Experimental 2017, 5(Suppl 1):P14

\section{Background}

Ventilator associated pneumonia (VAP) is a serious complication of mechanical ventilation that has been shown to be associated with increased medical costs and mortality. Bacterial biofilms contribute significantly to the recalcitrant nature of VAP. Commercial silvercoated endotracheal tube (Commercial ETT) had been developed but limit using due to highly cost and using metallic nanoparticles. To create and investigate antibacterial and antibiofilm efficacy of silvercoated green synthesis endotracheal tube with reducing agent extracted from Eucalyptus (AgNPs ETT).

Methods

Silver nanoparticles and Eucalyptus reducing agents were prepared and coated on endotracheal tubes by electrostatic selfassembly technique. Three types of ETT were compared antibacterial and antibiofilm activity; AgNPs ETT, commercial ETTs and uncoated ETTs. Staphylococcus aureus, Pseudomonas aeruginosa and Actinobacter baumanni were inoculated to form biofilm. We used modified Kirby-Bauer method to identify the diameter of growth inhibition zone $(\mathrm{mm})$, optical density and biofilm formation method to detect antibacterial activity and bacterial adherence respectively. 
Results

AgNPs ETT was found to be most effective in inhibition of P.aeruginosa followed by commercial ETT and uncoated ETT (4.0 (interquartile range (IQR) 2.2 to 5.0 versus $3.5, \mathrm{IQR} 2.6$ to $4.4, \mathrm{P}=0.02$ ). This effect also found on S.aureus (3.5, IQR 3.0 to 4.6 versus 3.5 , IQR 2.3 to $4.0, \mathrm{P}=0.02$ ) and A.baumanii (4.0, IQR 4.0 to 4.4 versus 3.0 , IQR 2.6 to $3.0, P<0.01$ ). In biofilm formation testing, AgNPs ETT had insignificant bacterial logarithm colony forming unit (log CFU/ $\mathrm{ml}$ ) when compared with commercial ETT but better than uncoated ETT. Conclusions

Silver-coated green synthesis ETT could inhibit bacterial growth and biofilm formation of $P$. aeruginosa, S.aureus and A.baumanii in vitro. Keywords: Antibiofilm, silver nanoparticle, endotracheal tube, Eucalyptus

\section{Acknowledgements}

This study shows no conflict of interest.

\section{P15}

Ferritin heavy chain controls glucose metabolism and affords disease tolerance in sepsis

Sebastian Weis ${ }^{1,2}$, Ana Rita Carlos ${ }^{1}$, Maria Raquel Moita', Sumnima Singh ${ }^{1}$ Birte Blankenhaus ${ }^{1}$, Silvia Cardoso', Rasmus Larsen', Sofia Rebelo', Sascha Schäuble ${ }^{3}$, Laura Del Barrio ${ }^{1}$, Gilles Mithieux ${ }^{4}$, Fabienne Rajas ${ }^{4}$, Sandro Lindig ${ }^{2,5}$, Michael Bauer ${ }^{2,5}$, Miguel P. Soares ${ }^{1}$

${ }^{1}$ Instituto Gulbenkian de Ciência, Oeiras, Portugal; ${ }^{2}$ Department of Anesthesiology and Intensive Care Medicine, Jena University Hospital, Jena, Germany ${ }^{3}$ Language \& Information Engineering Laboratory, Friedrich-Schiller-University, Jena, Germany; ${ }^{4}$ INSERM U1213, Université Claude Bernard Lyon, France, 5Center for Sepsis Control and Care, Jena University Hospital, Jena, Germany

Correspondence: Sebastian Weis (sebastian.weis@med.uni-jena.de) Intensive Care Medicine Experimental 2017, 5(Suppl 1):P15

\section{Background}

Sepsis results from maladaptive immune and metabolic responses to infection compromising host homeostasis[1, 2]. Infected organisms use 2 distinct defense mechanisms against infections, i.e. resistance and disease tolerance [3]. Resistance mechanisms aim at reducing the number of pathogens. Disease tolerance is a preserves host homeostasis, without exerting a direct negative impact on pathogens[3]. Instead, it relies on tissue damage control that provide metabolic adaptation and preserves the functional output of parenchymal tissues, maintaining homeostatic parameters within a dynamic range compatible with host survival[4]. Previously, it was shown that expression of the gene encoding the heavy/heart subunit of the iron sequestering molecule ferritin (Fth) is essential for the establishment of disease tolerance in malaria [5] as well as to viral and fungal infections in plants[6]. We hypothesized that expression of Fth protects against the deleterious outcome of bacterial sepsis via affording disease tolerance to infection.

Methods

We applied genetic loss-of function studies in different knock-out vs. control mice (Mx1CreFth $\Delta / \Delta$, AlbCreFth $\Delta / \Delta$, LyzMCreFth $\Delta / \Delta$, AlbCreERT2G6Pc1 $\Delta / \Delta$, Tlr4-/- and Ifng-/-) as well as adenovirus-mediated gene overexpresion in C57B6 mice. Mice underwent low- and high grade cecal ligation and puncture (CLP) or peritoneal contamination and infection (PCI) or were administered heme. Survival, disease severity, gene expression, cytokine content, cardio-vascular function, organ damage/dysfunction were assessed. Transcriptomics and hepatic metabolomics performed and integrated in a fluxvariance analysis.

Results

Here, we provide experimental evidence that deletion of Fth in sepsis is deleterious and that expression of Fth in the liver is critical to control glucose metabolism and establish disease tolerance to sepsis. Deletion of Fth in sepsis does not cause overt organ damage but instead results in failed metabolic adaptation and increased glycolysis, i.e. a parenchymal Warburg effect. The protective effect acts via a mechanism that counters iron-driven oxidative inhibition of the liver Glucose-6-Phosphatase (G6Pase). This is required to prevent the development of septic hypoglycemia that would compromise disease tolerance to sepsis, hence overcoming its lethal outcome. In addition, application of apoferritin reduces sepsis mortality via enhancing disease tolerance infection even when given 6 hours after infection.

\section{Conclusions}

Disease tolerance to sepsis relies on a cross talk between host iron and glucose adaptive metabolic responses. Ferritin sustains endogenous hepatic gluconeogenesis. This maintains blood glucose levels in response to infection. The described pathways can be targeted pharmacologically providing a novel therapeutic approach against sepsis that acts independently of pathogens.

\section{Acknowledgements}

Grant: DFG WE 4971/3 to SW. SFRH/BPD/101608/2014 to ARC. GlioPATH (grant FKZ 01ZX1402C) to SAS. BMBF; grant 01 EO1002 and

Meta-ZIK (03Z2J52) to MB. e:Med initiative ERC-2011-AdG 294709DAMAGECONTROL, PTDC/SAU-TOX/116627/2010, HMSP-ICT/0022/2010 to MPS.

The full manuscript has been published: Metabolic Adaptation Establishes Disease Tolerance to Sepsis.

Weis S, Carlos AR, Moita MR, Singh S, Blankenhaus B, Cardoso S, Larsen R,

Rebelo S, Schäuble S, Del Barrio L, Mithieux G, Rajas F, Lindig S, Bauer M, Soares MP.

Cell. 2017 Jun 15;169(7):1263-1275.e14.

\section{References}

1. Singer $M$, Deutschman $C S$, Seymour CW, Shankar-Hari M, Annane $D$, Bauer M, Bellomo R, Bernard GR, Chiche JD, Coopersmith CM et al: The Third International Consensus Definitions for Sepsis and Septic Shock (Sepsis-3). Jama 2016, 315(8):801-810.

2. Kotas ME, Medzhitov R: Homeostasis, Inflammation, and Disease Susceptibility. Cell 2015, 160(5):816-827.

3. Medzhitov R, Schneider DS, Soares MP: Disease Tolerance as a Defense Strategy. Science 2012, 335:936-941.

4. Soares MP, Gozzelino R, Weis S: Tissue damage control in disease tolerance. Trends in immunology 2014, 35(10):483-494.

5. Gozzelino R, Soares MP: Coupling heme and iron metabolism via ferritin H chain. Antioxid Redox Signal 2013, 20(11):1754-1769.

6. Deak M, Horvath GV, Davletova S, Torok K, Sass L, Vass I, Barna B, Kiraly Z, Dudits D: Plants ectopically expressing the iron-binding protein, ferritin, are tolerant to oxidative damage and pathogens. Nature biotechnology 1999, 17(2):192-196.

\section{P16}

Intestinal Alkaline Phosphatase (IAP) enhances the gastrointestinal barrier function and modulates tight junction expression in an experimental model of polymicrobial sepsis

Philip Plaeke', Joris G De Man ${ }^{1}$, Annemieke Smet ${ }^{1}$, Hanne Van Spaendonk ${ }^{1}$, Sara Nullens ${ }^{1}$, Philippe G Jorens ${ }^{1,2}$, Guy Hubens ${ }^{3,4}$ Benedicte Y De Winter

${ }^{1}$ Laboratory of Experimental Medicine and Paediatrics, University of Antwerp, Wilrijk, Belgium; ${ }^{2}$ Department of Intensive Care Medicine, Antwerp University Hospital, Edegem, Belgium; ${ }^{3}$ Antwerp Surgical Training, Anatomy and Research Centre, University of Antwerp, Wilrijk, Belgium; ${ }^{4}$ Department of Abdominal Surgery, Antwerp University Hospital, Edegem, Belgium

Correspondence: Philip Plaeke (philip.plaeke@uantwerpen.be) Intensive Care Medicine Experimental 2017, 5(Suppl 1):P16

\section{Background}

Sepsis is a severe condition characterized by the activation of a systemic and intestinal inflammatory cascade and mucosal barrier dysfunction. Intestinal alkaline phosphatase (IAP), an endogenously expressed, brush-border-bound enzyme is ubiquitously produced in the mucosa of the small intestine. IAP was demonstrated to detoxify several proinflammatory molecules (e.g. LPS, UDP) and is presumed to play a role in the maintenance of the healthy intestinal microbiome. Additionally, recent clinical trials established a nephroprotective function of IAP when administered systemically in septic patients. This study aimed to investigate the effects of intraperitoneally administered IAP on the intestinal barrier function of the small intestine in a cecal ligation and puncture (CLP)-model for sepsis.

Methods

Male OF-1 mice were randomized into 4 groups ( $n=12$ /group): sham + vehicle, sham + IAP, CLP + vehicle or CLP + IAP. On the 
first day, mice received an intraperitoneal (ip) injection of calf IAP (1 IU/g mouse, New England Biolabs) or saline (vehicle) 5 min. prior to a CLP-procedure (50\% ligation, single $21 \mathrm{G}$ puncture) or a sham-laparotomy. Subsequently, ip injections of IAP or the vehicle were repeated twice a day. Analgesia and fluid resuscitation were regularly provided. A validated clinical disease score [1] and the body weight were monitored daily. On the 2 nd postoperative day, the abdomen was reopened, $100 \mu \mathrm{l}$ of $4 \mathrm{kDa}$ FITC-Dextran was injected directly in the distally ligated ileum and quantified one hour later by fluorescencespectrophotometry to measure intestinal permeability. Total RNA was extracted from an ileal bowel specimen and the expression of cytokine and tight junction genes was determined by RTPCR.

Results

Septic mice had significantly worse clinical disease scores, lost more weight and had increased intestinal permeability compared to their sham-operated counterparts. After the CLPprocedure, gene expression of IL1 $\beta$, IL6, Tnfa and Cldn14 were significantly elevated in both vehicle- and IAP-treated mice (Table 3).

Similarly, Occludin and Cldn2 gene expression decreased significantly. IAP-treatment did not result in better clinical outcomes. Nevertheless, IAP did significantly reduce the observed disturbances in intestinal permeability in septic animals (Fig. 10). Additionally, IAPtherapy significantly elevated the expression levels of Cldn1 and Cldn14.

\section{Conclusion}

In mice, CLP-induced sepsis is associated with enhanced intestinal permeability, modified expression of intestinal tight junctions and increased expression of ileal inflammatory markers. Treatment of septic mice with IAP significantly lowered the permeability of the intestinal barrier at the small bowel and elevated expression levels of Cldn1 and Cldn14. We expect this increase to be linked to the observed improvements of the intestinal barrier function after IAP-treatment.

\section{Reference}

1. Heylen M.: Colonoscopy and MPET/CT are Valid Techniques to Monitor Inflammation in the Adoptive Transfer Colitis Model in Mice. Inflamm Bowel Dis. 2013, 19:967-97.

Table 3 (abstract P16). See text for description

\begin{tabular}{lllll}
\hline & Sham + Vehicle & Sham + IAP & CLP + Vehicle & CLP + IAP \\
\hline Clinical follow-up & & & & \\
Clinical disease score & $0.00 \pm 0.00$ & $0.08 \pm 0.08$ & $5.17 \pm 0.32^{*}$ & $4.25 \pm 0.36^{*}$ \\
Baseline Weight (g) & $36.24 \pm 1.15$ & $35.04 \pm 1.40$ & $36.92 \pm 1.41$ & $36.39 \pm 1.32$ \\
Weight loss (\%) day 2 & $5.35 \pm 1.01$ & $2.65 \pm 1.43$ & $10.37 \pm 0.70^{*}$ & $10.55 \pm 0.92^{*}$ \\
Permeability & & & & \\
FITC-Dextran & $1.00 \pm 0.17$ & $0.79 \pm 0.11$ & $6.88 \pm 1.51^{*}$ & $3.45 \pm 0.62^{*} \#$ \\
Concentration (1) & & & & \\
PCR-results & & & & \\
IL1ß & $1.19 \pm 0.19$ & $1.15 \pm 0.23$ & $10.28 \pm 3.73^{*}$ & $7.45 \pm 2.35^{*}$ \\
IL6 & $1.27 \pm 0.27$ & $1.36 \pm 0.29$ & $8.84 \pm 3.26^{*}$ & $5.68 \pm 1.56^{*}$ \\
TNFa & $1.17 \pm 0.19$ & $1.17 \pm 0.13$ & $2.03 \pm 0.30^{*}$ & $2.85 \pm 0.47^{*}$ \\
Claudin-1 (Cldn1) & $1.27 \pm 0.16$ & $1.55 \pm 0.23 \#$ & $1.34 \pm 0.28$ & $2.42 \pm 0.43 \#$ \\
Claudin-2 (Cldn2) & $1.14 \pm 0.14$ & $1.17 \pm 0.12$ & $0.79 \pm 0.08^{*}$ & $0.84 \pm 0.12^{*}$ \\
Claudin-14 (Cldn14) & $1.13 \pm 0.24$ & $2.37 \pm 0.84 \#$ & $2.53 \pm 0.43^{*}$ & $5.28 \pm 1.56^{*} \#$ \\
Occludin & $1.22 \pm 0.17$ & $1.26 \pm 0.19$ & $0.68 \pm 0.10^{*}$ & $0.69 \pm 0.10^{*}$ \\
\hline
\end{tabular}

Results are expressed as mean \pm SEM (Standard error of mean)

(1) Relative FITC-concentration to the FITC-levels measured in

vehicle-treated sham-mice

* Significantly different from sham-operated mice

\# Significantly different from vehicle-treated mice

Statistics - Two Way Analysis of Variance with LSD Post-Hoc analysis when

appropriate, a p-value of 0.05 was considered statistically significant

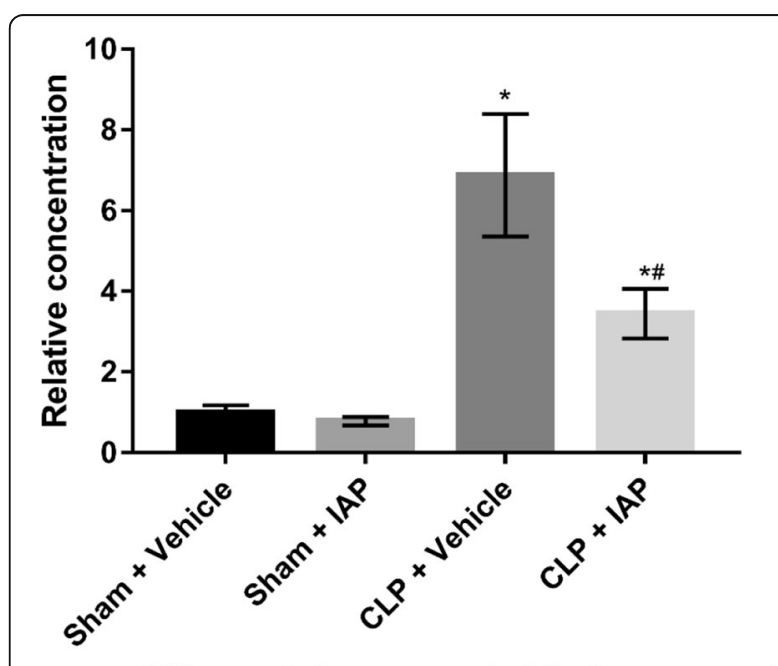

FITC-concentrations expressed relative to measurements in vehicle-treated sham-mice.

*Significantly different from sham-operated mice.

\#Significantly different from vehicle-treated mice

Fig. 10 (abstract P16). Permeability for FITC-Dextran (4 kDa) of the small intestine in a CLP-model for sepsis

P17

Possible mechanisms of dying of septic patients associated with aromatic microbial metabolites

NV Beloborodova', NI Fedotcheva², W Teplova², AK Pautova'

${ }^{1}$ Negovsky Research Institute of General Reanimatology, Federal

Research and Clinical Center of Intensive Care Medicine and

Rehabilitology, Petrovka, 25/2, Moscow, 107031, Russia; ${ }^{2}$ Institute of

Theoretical and Experimental Biophysics, Russian Academy of Sciences,

Institutskaya 3, Pushchino, Moscow region, 142290, Russia

Correspondence: NV Beloborodova (nvbeloborodova@yandex.ru)

Intensive Care Medicine Experimental 2017, 5(Suppl 1):P17

\section{Background}

The main causes of death in ICU are multiple organ failure (MOF) associated with sepsis and refractory septic shock[1]. Concept of mitochondrial dysfunction during sepsis is not new[2], but it remains unclear what role bacteria play. Our experimental approaches have revealed the influence of aromatic microbial metabolites (AMM) on some mitochondrial function[3]. We want to show how AMM is associated with mortality rates through the development of MOF and septic shock.

Methods

The study included adult non-survivors patients $(N=91)$, from which serum samples were taken and frozen for one or two days before the death. Serum samples from 20 donors and 24 survivors of sepsis were as comparison groups. The main equipment: GH-MS (Thermo Scientific) and GH-FID. All chemicals were from Sigma-Aldrich (US). Results

One day before death in $100 \%$ of cases only three AMM were increased, while other biochemical parameters of organs dysfunction in $9-40 \%$ of cases did not exceed normal values (Table 4). In the dynamics the closer the moment of death the increase of PhLA, p-HPhAA, pHPhLA were more significant than the growth of other routine parameters and severity scales (Table 5).

The serum levels of the mitochondrial metabolites - tricarboxylic acid cycle (TCA) were measured simultaneously with AMM. More than half of septic patients showed a multiple increase in succinic and fumaric acids, also the appearance in the blood of the alpha-ketoglutarate. This can be interpreted as a inhibition of the TCA. Correlations of mitochondrial metabolites (fumaric acid) with AMM $(r=0.68, p<0.01)$ and among themselves (fumaric with succinic) $(r=0.74, p<0.01)$ 
were noted. Direct correlation $(r=0.59, p<0.01)$ between $\Sigma 3$ AMM and presence of arterial hypotension was found. Only in septic shock patients but never in comparison groups the intermediate (DOPAC) and final (HVA) product of a minor pathway of tyrosine were detected in serum (Fig. 11). The microbial origin of these metabolites $[4,5]$ and their ability to inhibit the tyrosine hydroxylase are known[6,7]. The correlation between $\Sigma 3 \mathrm{AMM}$ and HVA reached a high rate, $r=0.76$.

Conclusions

AMM can be involved in dying of sepsis: 1) due the development of the MOF via the inhibition of TCA metabolism in the mitochondria and 2) through the mechanism of septic shock by inhibiting of the metabolic pathway of catecholamine synthesis, particularly tyrosine hydroxylase. The search in this direction will help to identify new therapeutic targets to improve the survival in sepsis.

\section{Acknowledgements}

Supported by Russian Science Foundation Grant №15-15-00110

\section{References}

1. Vincent $J L$, Nelson DR, Williams MD. Is worsening multiple organ failure the cause of death in patients with severe sepsis? Crit Care Med. 2011;39(5):1050-1055. doi:10.1097/CCM.0b013e31820eda29

2. Singer M. The role of mitochondrial dysfunction in sepsis-induced multiorgan failure. Virulence. 2014 Jan 1; 5(1): 66-72. PMCID: PMC3916385 DOl: 10.4161/viru.26907

3. Beloborodova N.V., Teplova V.V., Fedotcheva N.I. Monograph. "The role of microbial metabolites in mitochondrial dysfunction in sepsis" LAP Lambert Academic Publishing, Germany, 2013, 89 p. ISBN: 978-3659-43111-1

4. Martin M, Gibello A, Fernández J, Ferrer E, Garrido-Pertierra A. Catabolism of 3- and 4-hydroxyphenylacetic acid by Klebsiella pneumoniae. 1991 Mar;137(3):621-8

5. Sparnins VL, Chapman PJ, Dagley S. Bacterial degradation of 4hydroxyphenylacetic acid and homoprotocatechuic acid. J Bacteriol. 1974 Oct; 120(1):159-67

6. Laschinski, G., Kittner, B., Brautigam, M., 1986. Direct inhibition of tyrosine hydroxylase from PC12 cells by catechol derivaties. NaunynSchmiedeberg's Arch. Pharmacol. 3324, 346-350

7. Rong-Sen Shen. Potent inhibitory effects of tyrosine metabolites on dihydropteridine reductase from human and sheep liver. 1984, Biochimica et Biophysica Acta (BBA) - Protein Structure and Molecular Enzymology, 785, 3, 181-185

Table 4 (abstract P17). The frequency and severity of changes in the levels of aromatic microbial metabolites ( $\mathrm{p}$-HPhAA, PhLA, p-HPhLA) in comparison with other biochemical parameters of organ dysfunctions in septic patients in point of «the last day before death», median (IR 25-75\%)

\begin{tabular}{|c|c|c|c|}
\hline Parameter & $\begin{array}{l}\text { Donors, } \\
\mathrm{n}=20\end{array}$ & $\begin{array}{l}\text { Pts, the last day before } \\
\text { death, } n=91\end{array}$ & $\begin{array}{l}\text { The incidence of } \\
\text { abnormalities, \% }\end{array}$ \\
\hline${ }^{a} \mathrm{p}-\mathrm{HPhAA}, \mu \mathrm{M}$ & $0.87-2.53$ & $24.11(14.16-53.77)$ & 100 \\
\hline 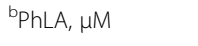 & $0.27-1.04$ & $7.23(5.10-17.00)$ & 99 \\
\hline${ }^{c} \mathrm{p}-\mathrm{HPhLA}, \mu \mathrm{M}$ & $0.50-2.27$ & $23.22(10.62-65.30)$ & 98 \\
\hline$\Sigma 3 \mathrm{AMM}, \mu \mathrm{M}$ & $1.80-5.15$ & $80.41(39.78-143.67)$ & 100 \\
\hline lactate, MM & $0.5-2.2$ & $5.6(3.3-10.0)$ & 91 \\
\hline urea, мM & $2.5-8.3$ & $22.3(14.7-32.2)$ & 90 \\
\hline creatinine, $\mu \mathrm{M}$ & $44-110$ & $257(173-348)$ & 88 \\
\hline total bilirubin, $\mathrm{MM}$ & $<18.8$ & $22.1(14,0-32.1)$ & 60 \\
\hline
\end{tabular}

${ }^{a} p-H P h A A$ p-hydroxyphenylacetic acid,

${ }^{\mathrm{b}} \mathrm{PhLA}$ phenyllactic acid,

${ }^{c} p$-HPhLA p-hydroxyphenyllactic acid
Table 5 (abstract P17). The increase in MODS score and some metabolites (median, IR 25-75\%) in septic patients during the last 48 hours before death $(n=34)$

\begin{tabular}{|c|c|c|c|c|}
\hline \multirow[t]{2}{*}{ Parameter } & \multicolumn{2}{|c|}{ Time to death (retrospective analysis) } & \multirow{2}{*}{$\begin{array}{l}\text { the multiplicity } \\
\text { of changes, Me }\end{array}$} & \multirow[t]{2}{*}{$p=$} \\
\hline & 48-25 hours & 24-0 hours & & \\
\hline $\mathrm{MODS}^{1}$ & $5(3-7)$ & $9(6-10)$ & 1,8 & $<0.001$ \\
\hline Lactate, mM & $3.6(2.4-5.1)$ & $4.2(3.0-10.6)$ & 1,2 & 0.03 \\
\hline PhLA, $\mu \mathrm{M}$ & $4.27(2.14-9.33)$ & $7.97(5.07-17.90)$ & 1,9 & $<0.001$ \\
\hline $\mathrm{p}$-HPhAA, $\mu \mathrm{M}$ & $10.45(1.99-25.81)$ & $20.14(7.27-65.49)$ & 1,9 & $<0.001$ \\
\hline p-HPhLA, $\mu \mathrm{M}$ & $9.14(5.73-25.51)$ & $22.06(13.64-67.04)$ & 2,4 & $<0.001$ \\
\hline$\sum 3 \mathrm{AMM}, \mu \mathrm{M}$ & $33.47(12.40-63.98)$ & $78.02(38.92-156.26)$ & 2,3 & $<0.001$ \\
\hline
\end{tabular}

${ }^{1}$ MODS - Multiple Organ Dysfunction Score

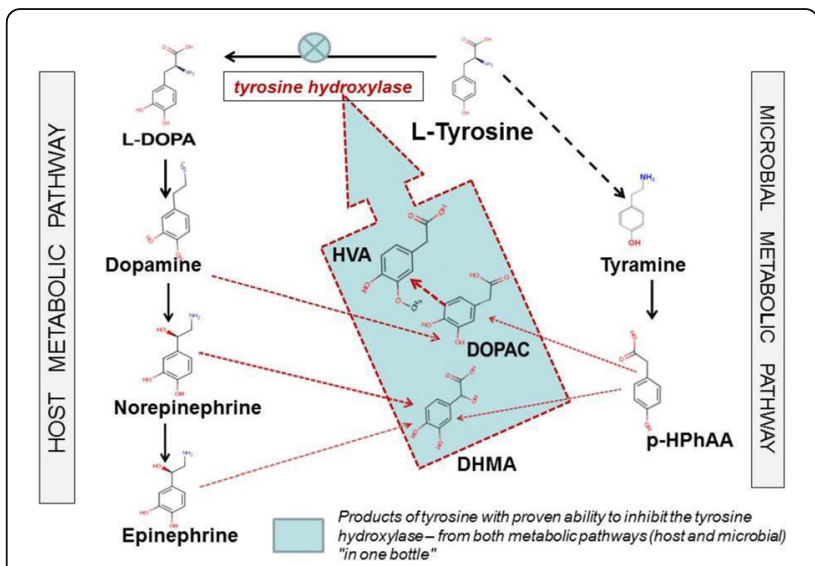

Fig. 11 (abstract P17). Inhibition of the metabolic pathway of catecholamine synthesis in sepsis

P18

Can base excess and anion gap predict lactate level in diagnosis of septic shock?

Werapon Pongmanee ${ }^{1}$, Veerapong Vattanavanit ${ }^{1}$

${ }^{1}$ Department of Internal Medicine, Faculty of Medicine, Prince of Songkla

University, Hat Yai, Songkhla, Thailand

Correspondence: Werapon Pongmanee

(sound_succeed@hotmail.com)

Intensive Care Medicine Experimental 2017, 5(Suppl 1):P18

\section{Background}

Lactate measurement is the key component in septic shock identification and resuscitation[1]. However, point-of-care lactate testing is not widely used due to lack of access. Biomarkers such as serum lactate, anion gap (AG), and base excess (BE) have been shown to be of use in determining shock in patients with seemingly normal vital signs.

Objective

To determine if these biomarkers can be used interchangeably in patients with septic shock in the emergency setting based on their test characteristics and correlation to each other.

Methods

A prospective observational cohort study was undertaken at a tertiary hospital in southern Thailand. Baseline point-of-care BE, AG, and serum lactate were recorded in all patients who presented with septic shock at the emergency department. Overall correlations including area under the receiver operating characteristic curve (AUROC) for both BE and AG to predict serum lactate level were calculated. 


\section{Results}

One hundred fifteen patients were enrolled. The median age was 66 years (interquartile range, 52-80 years), and 61\% were male. About thirty-five percent of septic shock were pneumonia. Pearson correlation of serum lactate to $B E$ was $-0.59(r 2=0.35$; $95 \%$ confidence interval $[\mathrm{Cl}],-0.69$ to $-0.44 ; P<.001)$, that of $\mathrm{BE}$ to $\mathrm{AG}$ was -0.67 $(\mathrm{r} 2=0.49 ; 95 \% \mathrm{Cl},-0.76$ to $-0.55 ; P<.001)$, and that for serum lactate to AG was 0.64 ( $\mathrm{r} 2=0.41 ; 95 \% \mathrm{Cl}, 0.52-0.74 ; P<.001)$ (Fig. 12). A cutoff point of AG 15.8 identified lactate level $\geq 2 \mathrm{mmol} / \mathrm{L}$ (sensitivity $71.4 \%$, specificity $80.7 \%, A U R O C 0.76$ ) and the best cutoff values to predict lactate level $\geq 4 \mathrm{mmol} / \mathrm{L}$ were 18.5 (sensitivity $64.2 \%$, specificity $85.5 \%$, AUROC 0.78 ) (Fig. 13).

\section{Conclusions}

In septic shock patients, lactate and AG showed strong correlation with each other; while, lactate and BE showed moderate correlation with each other. Thus, these biomarkers can be used interchangeably to help earlier determination of patients with septic shock.

Keywords: base excess, anion gap, lactate, septic shock

\section{Acknowledgements}

This study was fully funded by the Faculty of Medicine, Prince of Songkla University.

The authors report no conflicts of interest in this work.

\section{Reference}

1. Singer $M$, Deutschman CS, Seymour CW, Shankar-Hari M, Annane $D$ Bauer M, et al. The Third International Consensus Definitions for Sepsis and Septic Shock (Sepsis-3). Jama. 2016;315(8):801-10.
A

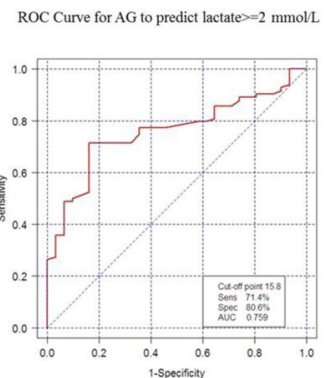

C

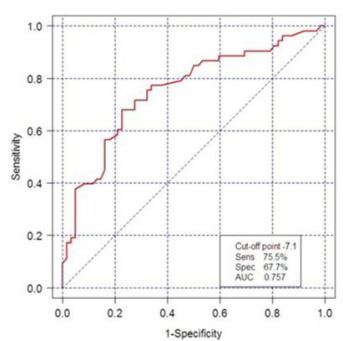

B

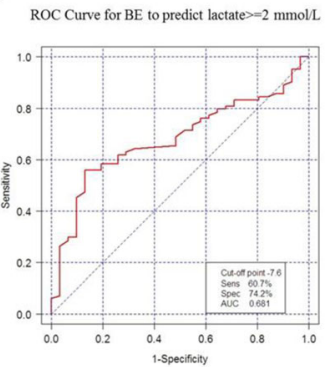

D

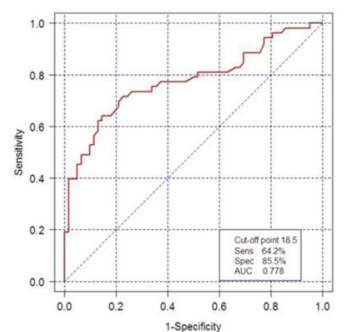

Fig. 13 (abstract P18). A-D,Reciever operator characteristic curve for AG plus BE to predict Lactate level $\geq 2$ and $\geq 4 \mathrm{mmol} / \mathrm{L}$

P19

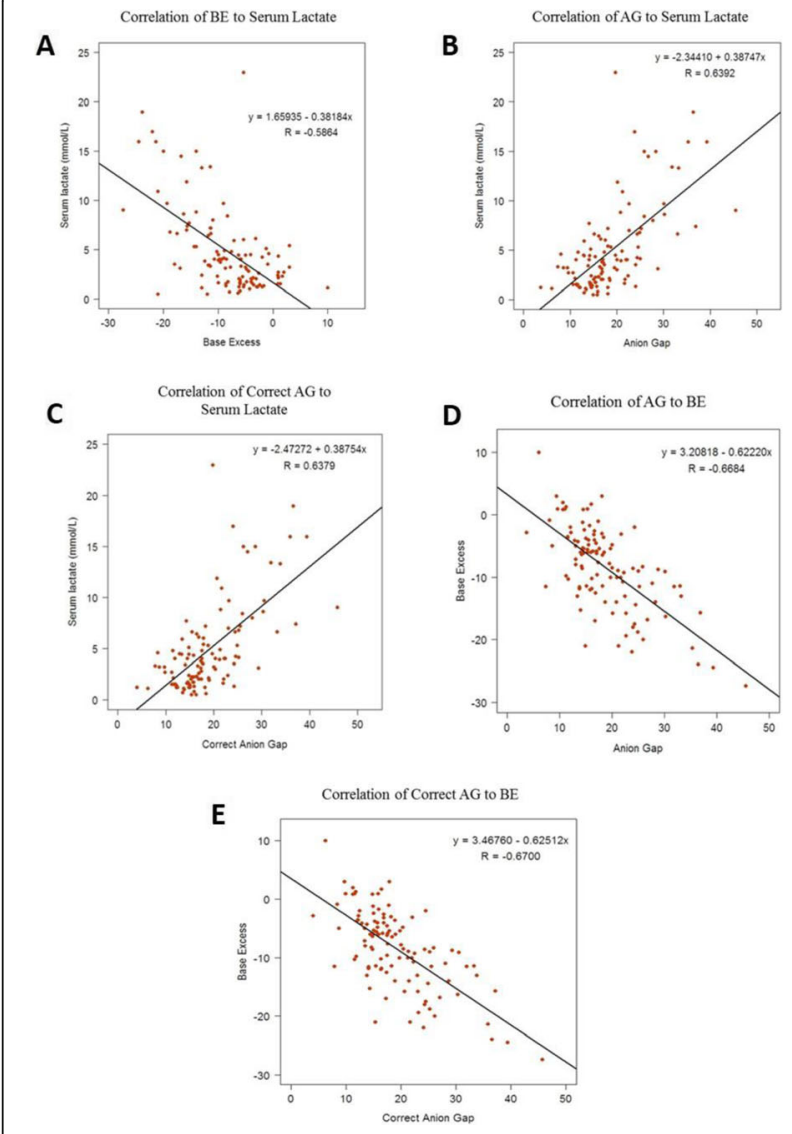

Fig. 12 (abstract P18). A-E, Correlation of (serum lactate to $B E, A G$, correct $A G)$ and (BE to $A G$, correct $A G)$
Cholecystokinin modulates the colonic inflammatory response and prevents epithelial barrier dysfunction in endotoxemic rats

Aline B. Ribeiro , Humberto Giusti', Rafael S. Saia

'Department of Physiology, Ribeirão Preto Medical School, University of São Paulo, Ribeirão Preto, São Paulo, Brazil

Correspondence: Rafael Simone Saia (rssaia@fmrp.usp.br)

Intensive Care Medicine Experimental 2017, 5(Suppl 1):P19

\section{Background}

The acute phase of sepsis is characterized by the exacerbated production of inflammatory mediators (chemokines, cytokines and nitric oxide). Especially the cytokines may contribute to the intestinal permeability, bacterial translocation and tight junction rearrangement. Cholecystokinin (CCK) was firstly described as a gastrointestinal hormone; however our research group has demonstrated an antiinflammatory involvement of this peptide in experimental models of endotoxemia and bacterial infection. Our aim was to determine the possible role of CCK on lipopolysaccharide (LPS)-induced intestinal epithelial barrier dysfunction. Furthermore, we evaluated its ability to modulate the mucosal inflammatory response and also the expression of proteins of the intestinal tight junctions.

Methods

To conduct these experiments, male rats had their jugular vein cannulated for endotoxin administration, one day before the experiment Rats were pre-treated with CCK ( 0.4 or $40 \mu \mathrm{g} / \mathrm{kg}$, intravenously) before LPS administration $(1.5 \mathrm{mg} / \mathrm{kg}$, intravenously). At $4 \mathrm{~h}$ after endotoxemia induction, the intestinal permeability was evaluated by injecting FITCdextran $4 \mathrm{kDa}$ in the ileum and colon; mesenteric lymph nodes were collected for microbiological analysis and also; cytokines were quantified in the plasma and intestinal mucosa by ELISA technique. Additionally, the integrity of the tight junctions was determined by the expression of its constituents proteins (occludin, claudin-1, junctional adhesion moleculeA) and the histological damages were evaluated in tissue sections.

Results

Our results demonstrated that CCK administration reduces the permeability only in the colon and prevented the bacterial translocation to the mesenteric lymph nodes. The levels of pro-inflammatory mediators TNF$a, \mathrm{LL}-1 \beta, \mathrm{IL}-6$ and IFN- $\gamma$ were reduced in CCK-treated rats, being in the colonic mucosa the most significant effect. Furthermore, treatment with 
CCK attenuated the LPS-induced epithelial barrier dysfunction, increasing the expression of occludin, claudin-1 and junctional adhesion moleculeA. Interestingly, the endotoxemia reduced only the expression of CCK-2 receptors in the colonic mucosa. Meanwhile, the CCK-treated rats showed higher expression of CCK-1 receptor in the mucosa, suggesting the possible mechanism of action for this peptide. Sections of the proximal colon did not evidence of histological damages or the presence of inflammatory cells infiltration.

\section{Conclusions}

Our present study sheds light on the fact that CCK treatment breaks the vicious cycle of local gut inflammation and barrier dysfunction in endotoxemia, especially preserving the integrity of one structure of this monolayer epithelium, the tight junctions.

\section{Acknowledgement}

FAPESP, CNPq and FAEPA.

\section{P20}

Superior accuracy of mid-regional proadrenomedullin over C reactive protein, procalcitonin and lactate for ICU mortality prediction in septic patients

Ana María Badía-Tejero', Fernando Martínez-Sagasti' ${ }^{2}$, Sara Domingo-Marín², Ma Ángeles del Dedo-Torre ${ }^{3}$, Mónica Requesens-Solera ${ }^{2}$,

Natalia González-Pereira ${ }^{3}$, Jorge Molina-del Pozo ${ }^{3}$, Christian Giersig-Heredia² Irene Cid-Tovar', Loreto López-Vergara ${ }^{2}$, Ma Dolores Ortega-De Heredia $^{3}$ ${ }^{1}$ Complutense University of Madrid, Madrid, Spain; ${ }^{2}$ Intensive Care Department, Hospital Clinico San Carlos, Madrid, Spain; ${ }^{3}$ Department of Laboratory Medicine, Hospital Clinico San Carlos, Madrid, Spain

Correspondence: Ana Maria Badia Tejero (anamatopeya@gmail.com)

Intensive Care Medicine Experimental 2017, 5(Suppl 1):P20

\section{Background}

Recently, studies of the potential usefulness of the mid-regional proadrenomedullin (MR-proADM) as mortality predictor in sepsis has provided interesting results[1,2 3,4]. The aim of our study was to evaluate the accuracy of MR-proADM to predict ICU mortality in septic patients compared with other more frequently used biomarkers, procalcitonin (PCT), C reactive protein (CRP) and lactate, and APACHE II and SOFA scores. Methods

This was a single-centre, prospective, observational, cohort, blind study, enrolling septic patients admitted to the ICU of a teaching tertiary hospital between November 2016 and April 2017. Blood samples were taken within the first 18 hours of ICU admission. The accuracy of the biomarkers and risk scores for ICU mortality was evaluated by calculating the area under the receiver operating characteristic (AUROC) curve. Survival was assessed by Kaplan Meier and COX regression analysis.

\section{Results}

Thirty-three consecutive patients with sepsis $(20.2 \%)$ or septic shock (78.8\%) were enrolled. The ICU mortality was $27.3 \%$. The mean age was 69.9 years (standard deviation [SD], 11.6) and $66.7 \%$ of patients were male. MR-proADM and lactate plasmatic levels were both significantly higher in non-survivors: $16.89 \mathrm{nmol} / \mathrm{L}(8.86-27.44)$ versus $3.83 \mathrm{nmol} / \mathrm{L}(1.04-8.86)(p$ $<0.001)$ and $5.56 \mathrm{mmol} / \mathrm{L}(1.50-10.50)$ versus $2.35 \mathrm{mmol} / \mathrm{L}(0.50-9.10)(p=$ $0.007)$, respectively. Non-surviving patients also had both a higher APACHE II score (31 [24-35] versus 20.5 [10-42]) $(p=0.004)$ and SOFA score (11 [8$14]$ versus $8[1-15])(p=0.032)$. Although plasmatic levels of CRP and PCT were higher in the patients who died, this effect was not statistically significant (Table 6). MR-proADM showed the best AUROC for ICU mortality prediction: $0.99(0.98-1 ; 95 \% \mathrm{Cl})(p<0.001)$. CRP levels were not associated with ICU mortality (Fig. 14 and Table 7). An optimal cut-off point of $8.58 \mathrm{nmol} / \mathrm{L}$ for MR-proADM was selected since it provided a sensitivity of $100 \%$ and specificity of $95.5 \%$. Kaplan Meier analysis showed that no patients with a MR-proADM value under this cut-off point died during their stay in the ICU (Fig. 15). Only MR-proADM and APACHE II were associated with ICU mortality in the multivariate analysis with a hazard ratio of 1.72 and 2.95 (both $p<0.05$ ), respectively (Table 8).

\section{Conclusions}

A unique determination of MR-proADM plasmatic levels in septic patients measured within the first 18 hours of admission in the ICU has better accuracy for ICU mortality prediction than CRP, PCT, lactate or APACHE II and SOFA scores.

\section{Acknowledgements}

The authors would like to thank the ICU nursing team and the lab staff for their contribution to this study collecting and processing the samples. They also thank Irene Serrano for helping with the statistical analysis and Dr Miguel Sánchez Garcia, head of the ICU for his advice interpreting the results. Finally, they would like to thank ThermoFisher for providing reagents for the MR-proADM evaluation.

\section{References}

1. Andaluz-Ojeda D, Cicuendez R, Calvo D., Nogales L, Muñoz M-F, Bueno P, Eiros J-M \& Gandía F. Sustained value of proadrenomedullin as mortality predictor in severe sepsis. J Infect 2015; 71:136-139.

2. Andaluz-Ojeda D., Nguyen H-B., Meunier-Beillard N., Cicuéndez R., Quenot J., Calvo D., Dargent A., Zarca E., Andrés C., Nogales L. et al. Superior accuracy of mid-regional proadrenomedullin for mortality prediction in sepsis with varying levels of illness severity. Ann. Intensive Care 2017; 7:15

3. Marino R, Struck J, Maisel A-S, Magrini L, Bergmann A \& Di Somma S. Plasma adrenomedullin is associated with short-term mortality and vasopressor requirement in patients admitted with sepsis. Crit Care 2014; 18:R34.

4. Valenzuela-Sanchez F, Valenzuela-Mendez B, Rodriguez-Gutierrez F, Estella-García A \& González-García MA. New role of biomarkers: midregional pro-adrenomedullin, the biomarker of organ failure. Ann Trans Med 2016

Table 6 (abstract P20). Characteristics of the patients

\begin{tabular}{|c|c|c|c|c|}
\hline & $\begin{array}{l}\text { Survivors } \\
(n=24)\end{array}$ & $\begin{array}{l}\text { Non-survivors } \\
(\mathrm{n}=9)\end{array}$ & $\begin{array}{l}\text { Total } \\
(n=33)\end{array}$ & $p$ \\
\hline \multicolumn{5}{|l|}{ Demographics } \\
\hline $\begin{array}{l}\text { - Patients } \\
(n, \%)\end{array}$ & $24(72.7)$ & $9(27.3)$ & $33(100)$ & \\
\hline $\begin{array}{r}- \text { Male } \\
(n, \%)\end{array}$ & $17(70.8)$ & $5(55.6)$ & $22(66.7)$ & 0.438 \\
\hline - Age, years (mean, SD) & $69.3(12.5)$ & $71.8(9.3)$ & $69,9(11.6)$ & 0.586 \\
\hline - BMI, kg/m² (mean, SD) & $25.6(7.1)$ & $25.1(4.4)$ & $25.5(6.4)$ & 0.848 \\
\hline \multicolumn{5}{|l|}{ Origin } \\
\hline $\begin{array}{l}\text { - Emergency department } \\
(n, \%)\end{array}$ & $7(29.2)$ & $3(33.3)$ & $10(30.3)$ & 0.644 \\
\hline - Ward (n, \%) & $4(16.7)$ & $3(33.3)$ & $7(21.2)$ & \\
\hline - Surgery (n, \%) & $12(50)$ & $3(33.3)$ & $15(45.5)$ & \\
\hline - Other hospital (n, \%) & $1(4.2)$ & - & $1(3)$ & \\
\hline \multicolumn{5}{|l|}{ Comorbidities } \\
\hline $\begin{array}{l}\text { - High blood } \\
\text { pressure (n, \%) }\end{array}$ & $12(50)$ & $8(88.9)$ & $20(60.6)$ & 0.056 \\
\hline $\begin{array}{l}\text { - Cardiovascular } \\
\text { disease }(n, \%)\end{array}$ & $9(37.5)$ & $4(44.4)$ & $13(39.4)$ & 1.000 \\
\hline - COPD (n, \%) & $6(25)$ & $1(11.1)$ & $7(21.2)$ & 0.642 \\
\hline $\begin{array}{l}\text { - Chronic kidney } \\
\text { disease }(n, \%)\end{array}$ & $5(20.8)$ & $1(11.1)$ & $6(18.2)$ & 1.000 \\
\hline $\begin{array}{l}\text { - Chronic liver } \\
\text { disease (n, \%) }\end{array}$ & $3(12.5)$ & $0(0)$ & $3(9.1)$ & 0.545 \\
\hline - Diabetes (n, \%) & $5(20.8)$ & $5(55.6)$ & $10(30.3)$ & 0.090 \\
\hline - Cancer (n, \%) & $6(25)$ & $8(88.9)$ & $14(42.4)$ & 0.002 \\
\hline $\begin{array}{l}\text { - Immunosuppression } \\
(\mathrm{n}, \%)\end{array}$ & $3(12.5)$ & $1(11.1)$ & $4(12.1)$ & 1.000 \\
\hline \multicolumn{5}{|l|}{ Infectious origin } \\
\hline - Respiratory (n, \%) & $11(45.8)$ & $2(22.2)$ & $13(39.4)$ & 0.329 \\
\hline - Abdominal (n, \%) & $11(45.8)$ & $5(55.6)$ & $16(48.5)$ & \\
\hline - Urinary (n, \%) & $1(4.2)$ & $1(11.1)$ & $2(6.1)$ & \\
\hline - Septic arthritis (n, \%) & $1(4.2)$ & - & $1(3)$ & \\
\hline - Soft tissues (n, \%) & - & $1(11.1)$ & $1(3)$ & \\
\hline
\end{tabular}


Table 6 (abstract P20). Characteristics of the patients

\section{(Continued)}

\begin{tabular}{|c|c|c|c|c|}
\hline \multicolumn{5}{|l|}{ Clinical variables } \\
\hline $\begin{array}{l}\text { - MAP, mmHg } \\
\text { (median, IQR) }\end{array}$ & $65.8(45-80)$ & $52(42-59)$ & $59(54.5-67)$ & $<0.001$ \\
\hline $\begin{array}{l}\text { - Heart rate, bpm } \\
\text { (median, IQR) }\end{array}$ & $113(68-147)$ & $148(115-170)$ & $120(104-134,5)$ & $<0.001$ \\
\hline $\begin{array}{c}\text { - } \text { Temperature, }{ }^{\circ} \mathrm{C} \\
\text { (median, IQR) }\end{array}$ & $37.1(35-41)$ & $37,8(35.1-38.5)$ & $37,2(35.8-38.3)$ & 0.858 \\
\hline $\begin{array}{l}\text { - Respiratory rate, } \\
\text { rpm (median, IQR) }\end{array}$ & $20(6-40)$ & $26(16-38)$ & $23(15-29)$ & 0.036 \\
\hline $\begin{array}{l}\text { - Glasgow score, } \\
\text { points (median, IQR) }\end{array}$ & $14(6-15)$ & $13(11-15)$ & $14(12-15)$ & 0.890 \\
\hline \multicolumn{5}{|l|}{ Laboratory variables } \\
\hline $\begin{array}{l}\text { - MR-proADM, nmol/L } \\
\text { (median, IQR) }\end{array}$ & $3.83(1.04-8.86)$ & $\begin{array}{l}16.89(8.86- \\
27.44)\end{array}$ & $4.98(3.22-8.72)$ & $<0.001$ \\
\hline $\begin{array}{l}\text { - Lactato, mmol/L } \\
\text { (median, IQR) }\end{array}$ & $2.35(0.50-9.10)$ & $5.56(1.50-10.50)$ & $3.20(1.50-4.25)$ & 0.007 \\
\hline $\begin{array}{l}\text { - PCT, ng/mL } \\
\quad \text { (median, IQR) }\end{array}$ & $\begin{array}{l}3.90(0.20- \\
118.70)\end{array}$ & $\begin{array}{l}29.79(2.40- \\
74.20)\end{array}$ & $\begin{array}{l}6.30(1.80- \\
35.05)\end{array}$ & 0.064 \\
\hline $\begin{array}{l}-\mathrm{CRP}, \mathrm{mg} / \mathrm{dL} \\
\text { (median, IQR) }\end{array}$ & $\begin{array}{l}16.30(1.65- \\
43.70)\end{array}$ & $\begin{array}{l}25.43(6.88- \\
49.70)\end{array}$ & $\begin{array}{l}17.35(10.1- \\
26.2)\end{array}$ & 0.228 \\
\hline $\begin{array}{l}\text { - White blood } \\
\text { cells, } \times 10^{3} / \mathrm{L} \\
\text { (median, IQR) }\end{array}$ & $\begin{array}{l}14.40(0,32- \\
35,00)\end{array}$ & $9.40(0.10-23.70)$ & $11.9(4.1-19.1)$ & 0.207 \\
\hline $\begin{array}{l}\text { - Platelets, } \times 10^{3} / \mathrm{L} \\
\text { (median, IQR) }\end{array}$ & $185.50(58-401)$ & $125(10-535)$ & $173(107.5-310)$ & 0.414 \\
\hline $\begin{array}{l}\text { - Creatinine, mg/dL } \\
\text { (median, IQR) }\end{array}$ & $1.13(0.35-4.10)$ & $2.30(0.21-6.09)$ & $1.6(0.9-2.2)$ & 0.032 \\
\hline \multicolumn{5}{|l|}{ Severity scores } \\
\hline $\begin{array}{l}\text { - APACHE II, points } \\
\text { (median, IQR) }\end{array}$ & $20.5(10-42)$ & $31(24-35)$ & $24(17-30)$ & 0.004 \\
\hline $\begin{array}{l}\text { - SOFA, points } \\
\text { (median, IQR) }\end{array}$ & $8(1-15)$ & $11(8-14)$ & $9(7-10,5)$ & 0.032 \\
\hline \multicolumn{5}{|l|}{ Other } \\
\hline $\begin{array}{l}\text { - Septic shock upon } \\
\text { ICU admission (n, \%) }\end{array}$ & $17(70.8)$ & $9(100)$ & $26(78.8)$ & 0.149 \\
\hline $\begin{array}{l}\text { - ICU stay, } \\
\text { days (median, IQR) }\end{array}$ & $7.5(2-31)$ & $15(2-46)$ & $9(3-17)$ & 0.193 \\
\hline - ICU mortality (n, \%) & & & $9(27.3)$ & \\
\hline - 28 day mortality (n, \%) & & & $14(42.4)$ & \\
\hline
\end{tabular}

$B M I$ body mass index, COPD chronic obstructive pulmonary disease, $T B C$ tuberculosis, $P C R$ polymerase chain reaction, IQR interquartilic range, IMV invasive mechanical ventilation, SOFA sequential [sepsis related] organ failure assessment

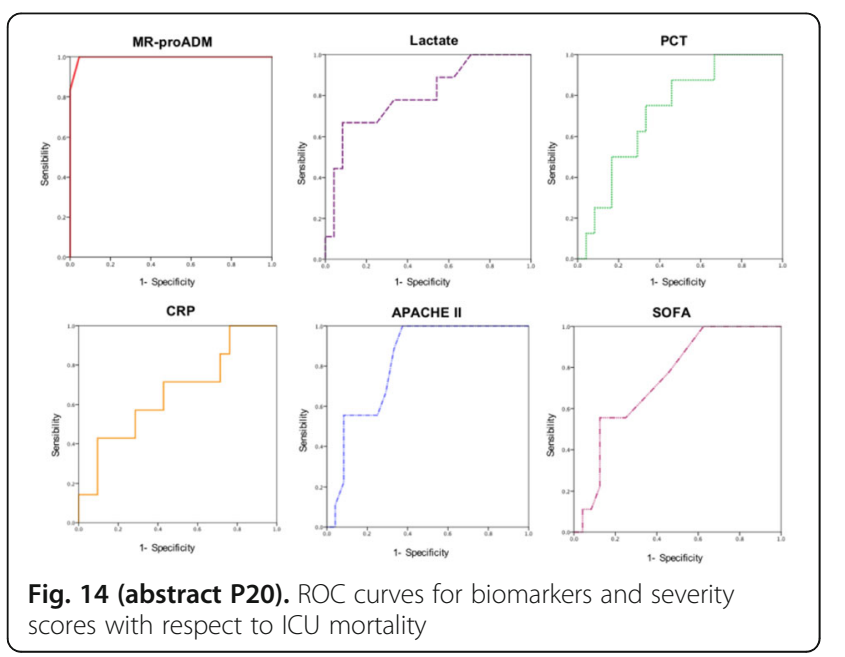

Table 7 (abstract P20). AUROC analysis for ICU mortality prediction

\begin{tabular}{llllllll}
\hline Biomarker & AUC $(95 \% \mathrm{Cl})$ & $p$ & Cutt-off point & Se \% & Sp \% & PPV \% & NPV \% \\
\hline MR- & $0.99(0.98-1.00)$ & $<0.001$ & 8.58 & 100 & 95.5 & 85.7 & 100 \\
proADM & & & & & & & \\
Lactate & $0.80(0.63-0.98)$ & 0.009 & 3.35 & 77.8 & 66.7 & 46.7 & 88.9 \\
PCT & $0.72(0.54-0.91)$ & 0.061 & 4.10 & 87.5 & 458 & 38.9 & 92.9 \\
CRP & $0.66(0.42-0.90)$ & 0.212 & 17.35 & 71.4 & 57.1 & 36.8 & 85.7 \\
APACHE II & $0.82(0.68-0.96)$ & 0.005 & 23.00 & 100 & 62.5 & 50 & 100 \\
SOFA & $0.74(0.57-0.92)$ & 0.034 & 8.50 & 77.8 & 54.2 & 38.9 & 86.7
\end{tabular}

AUC Area Under The Curve, Se sensibility, Sp specificity, PPV positive predictive value, $N P V$ negative predictive value

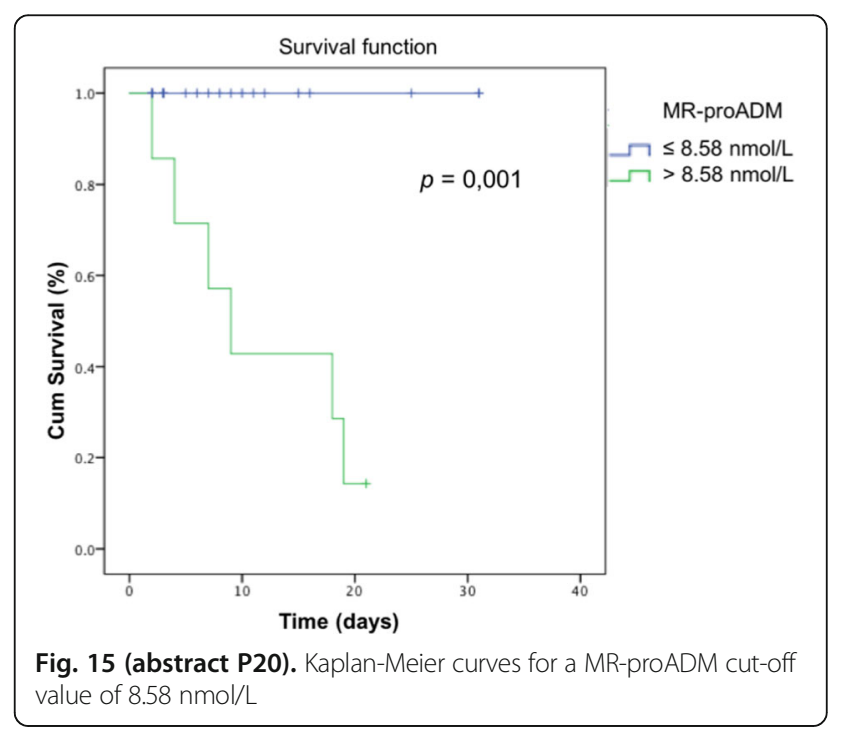

Table 8 (abstract P20). Univariate and multivariate COX regression analysis for ICU mortality prediction

\begin{tabular}{llllll}
\hline & \multicolumn{1}{l}{ Univariate } & & \multicolumn{2}{l}{ Multivariate } \\
\cline { 2 - 3 } & HR $(95 \% \mathrm{Cl})$ & $p$ & & HR $(95 \% \mathrm{Cl})$ & $p$ \\
\hline MR-proADM & $1.12(1.03-1.22)$ & 0.012 & & $1.72(1.02-2.92)$ & 0.043 \\
Lactate & $1.45(1.06-2.00)$ & 0.022 & & $1.37(0.88-2.14)$ & 0.160 \\
PCT & $1.01(0.98-1.03)$ & 0.677 & & - & - \\
CRP & $1.03(0.97-1.10)$ & 0.311 & & - & - \\
APACHE II & $1.18(1.04-1.34)$ & 0.011 & & $2.95(1.01-8.59)$ & 0.048 \\
SOFA & $1.28(0.97-1.69)$ & 0.083 & & $0.09(0.01-1.02)$ & 0.052 \\
\hline
\end{tabular}

P21

Cardiac overexpression of Melusin protects from LPS-induced sepsis cardiomyopathy

Pietro Arina', Giuseppe Rotondo', Nicoletta Vitale², Andrea Costamagna', Paola Cappello ${ }^{3}$, Lorenzo Del Sorbo ${ }^{4}$, Anna Mazzeo ${ }^{1}$, Luciana Mascia ${ }^{5}$, Luca Brazzi ', Vito Marco Ranieri' ${ }^{6}$, Fiorella Altruda ${ }^{2}$, Mara Brancaccio ${ }^{2}$, Vito Fanelli ${ }^{1}$ ${ }^{1}$ Department of Anesthesia and Critical Care - AOU Città della Salute e della Scienza di Torino - University of Turin, Turin, Italy; ${ }^{2}$ Department of Molecular Biotechnology and Health Sciences - University of Turin, Turin, Italy; ${ }^{3}$ CeRMS-Lab di Immunologia dei Tumori - University of Turin, Turin, Italy; ${ }^{4}$ Department of Medicine, Division of Respirology and Critical Care Medicine, Toronto General Hospital - University Health Network and Mount Sinai Hospital, Toronto, Canada; ${ }^{5}$ Department of Medico-Surgical Sciences and Biotechnologies, University of Rome Sapienza University of Rome, Rome, Italy; ${ }^{6}$ Department of Anesthesia and Intensive Care Medicine - Policlinico Umberto I Hospital - Sapienza University of Rome, Rome, Italy Correspondence: Pietro Arina (pietro.arina@gmail.com) Intensive Care Medicine Experimental 2017, 5(Suppl 1):P21 


\section{Background}

The aim of this work was to assess the role of Melusin in a model of LPSinduced Sepsis cardiomyopathy (SIC). Melusin is a muscle-specific chaperone protein involved in sustaining cardiomyocyte survival and adaptive hypertrophy in different stress conditions thorough the activation of ERK1/2 and AKT signaling pathways[1,2,3,4,5]. Accordingly, Melusin levels increase during the compensatory hypertrophy response to stress and decrease during later stages of heart failure[6, 7]. However, its role in LTCC dysfunction associated with sepsis is unknown. We hypothesized that Melusin overexpression could exert a protective effect on cardiac function during septic injury through the increased phosphorilation of AKT, a protein involved in the control of LTCC density in heart cells[8]. An experimental model of septic pre-clinical disease by comparing two different strains of mice have been developed, either with Knockout mice (KO) for Melusin gene (SV129 strain) or with cardiac-specific overexpression (OV) of Melusin (FVB strain). Each group was studied together with a control group.

\section{Methods}

Animals were treated with an intraperitoneal injection of lipopolysaccharide (LPS) at $12 \mathrm{mg} / \mathrm{kg}$ to induce a septic state at 0 hours.

Measurements and Main Results: At 0, 6 and 24 hours were recorded in both groups, cardiac and hemodynamic parameters through echocardiography and non-invasive detection of blood pressure. Another cohort of animals was sacrificed 6 hours after $20 \mathrm{mg} / \mathrm{kg}$ LPS treatment and cardiac tissues extracts were harvested for Western Blot analysis to quantify the expression of AKT, P-AKT and CACNA1C. Results

In both SV129 WT and KO Melusine mice, fractional shortening (FS) was significantly impaired after LPS challenge and was associated with compensatory tachycardia (Fig. 16). After 6 and 24 hours from LPS challenge, in Melusine KO mice, systolic, diastolic and mean arterial pressure were significantly lower than baseline. In Melusin WT mice, these differences were significant only at 24 hours (Fig. 17). FVB WT mice FS was significantly impaired after LPS challenge and was associated with compensatory tachycardia.In OV mice the tachycardia was also present at 6 hours after LPS challenge. Comparing the two groups, OV mice significantly differ for fractional shortening and heart rate at 6 hours after LPS challenge (Fig. 18).

Consistent with the increased AKT phosphorylation observed in OV mice, the expression of CACNA1C was also significantly higher both at basal levels and after LPS treatment in OV mice compared to WT mice (Fig. 20). Conclusions

Melusin has protective role in LPS induced cardiomyopathy, likely through Akt phosphorylation controlling the CACNA1C protein density.

\section{References}

1. Unsöld, Bernhard, Axel Kaul, Mauro Sbroggiò, Carola Schubert, Vera RegitzZagrosek, Mara Brancaccio, Federico Damilano, et al. 2014. "Melusin Protects from Cardiac Rupture and Improves Functional Remodelling after Myocardial Infarction." Cardiovascular Research 101 (1): 97-107. doi:10.1093/cvr/cvt235.

2. Penna, Claudia, Mara Brancaccio, Francesca Tullio, Cristina Rubinetto, Maria-Giulia Perrelli, Carmelina Angotti, Pasquale Pagliaro, and Guido Tarone. 2014. "Overexpression of the Muscle-Specific Protein, Melusin, Protects from Cardiac Ischemia/Reperfusion Injury." Basic Research in Cardiology 109 (4): 418. doi:10.1007/s00395-014-0418-9.

3. Sbroggiò, Mauro, Alessandro Bertero, Silvia Velasco, Federica Fusella, Emanuele De Blasio, Wadie F. Bahou, Lorenzo Silengo, Emilia Turco, Mara Brancaccio, and Guido Tarone. 2011. "ERK1/2 Activation in Heart is Controlled by Melusin, Focal Adhesion Kinase and the Scaffold Protein IQGAP1." Journal of Cell Science 124 (Pt 20): 3515-24. doi:10.1242/jcs.091140.

4. Brancaccio, Mara, Luigi Fratta, Antonella Notte, Emilio Hirsch, Roberta Poulet, Simona Guazzone, Marika De Acetis, et al. 2003. "Melusin, a Muscle-Specific Integrin beta1-Interacting Protein, Is Required to Prevent Cardiac Failure in Response to Chronic Pressure Overload." Nature Medicine 9 (1): 68-75. doi:10.1038/nm805.

5. De Acetis, Marika, Antonella Notte, Federica Accornero, Giulio Selvetella, Mara Brancaccio, Carmine Vecchione, Mauro Sbroggiò, et al. 2005. "Cardiac Overexpression of Melusin Protects from Dilated Cardiomyopathy due to Long-Standing Pressure Overload." Circulation Research 96 (10): 1087-94. doi:10.1161/01.RES.0000168028.36081.e0.

6. Sorge, Matteo, and Mara Brancaccio. 2016. "Melusin Promotes a Protective Signal Transduction Cascade in Stressed Hearts." Frontiers in Molecular Biosciences 3: 53. doi:10.3389/fmolb.2016.00053.
7. Tarone, Guido, and Mara Brancaccio. 2015. "The Muscle-Specific Chaperone Protein Melusin Is a Potent Cardioprotective Agent." Basic Research in Cardiology 110 (2): 10. doi:10.1007/s00395-015-0466-9.

8. Catalucci, Daniele, Deng-Hong Zhang, Jaime DeSantiago, Franck Aimond, Guillaume Barbara, Jean Chemin, Désiré Bonci, et al. 2009. "Akt Regulates L-Type Ca2+ Channel Activity by Modulating Cavalpha1 Protein Stability." The Journal of Cell Biology 184 (6): 923-33. doi:10.1083/jcb.200805063.
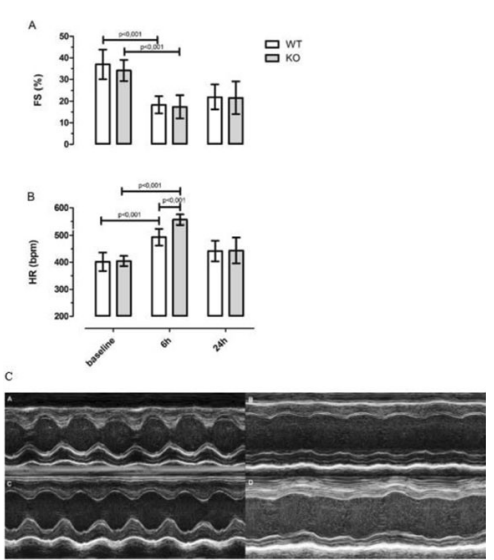

Fig. 16 (abstract P21). Panel A Changes in Fractional Shortening (FS) versus time. Experimental groups are: SV129 WT, SV129 KO for Melusin expression, where $\mathrm{n}=12$ for both groups. Panel $\mathrm{B}$ Changes in Heart Rate $(\mathrm{HR})$ versus time. The groups were the same in A. Panel C: A Mmode echography of Left Ventricle (LV) at baseline of SV129 WT mice. B M-mode echography of LV after $6 \mathrm{~h}$ of LPS challenge of SV129 WT mice. C M-mode echography of LV at baseline of SV129 KO mice. D M-mode echography of LV after $6 \mathrm{~h}$ of LPS challenge of SV129 KO mice
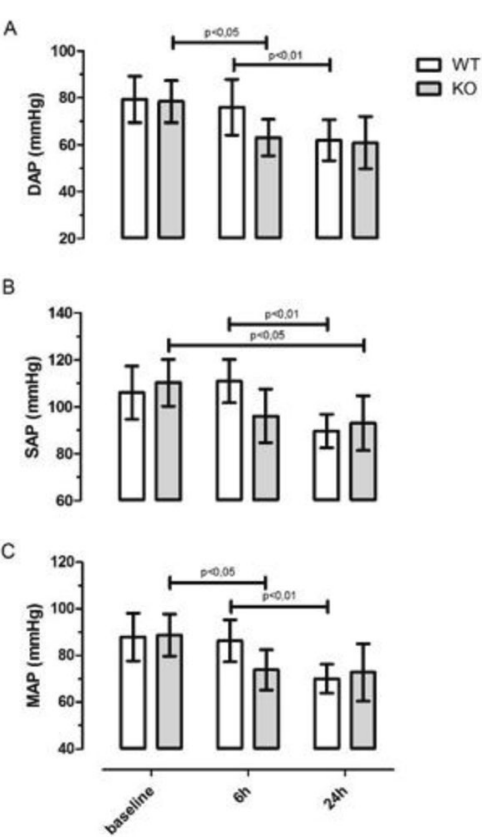

Fig. 17 (abstract P21). Panel A Changes in Diastolic Arterial Pressure (DAP) versus time. Experimental groups are: SV129 WT, SV129 KO for Melusin Expression, where $n=10$ for both groups. Panel B Changes in Systolic Arterial Pressure (SAP) versus time. Experimental groups are the same in $\mathrm{A}$. Panel $\mathrm{C}$ Changes in Mean Arterial Pressure (MAP) versus time. The groups are the same in A 

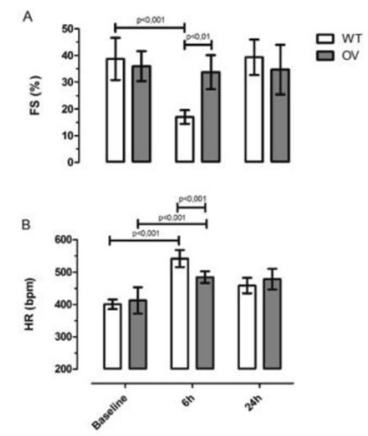

c

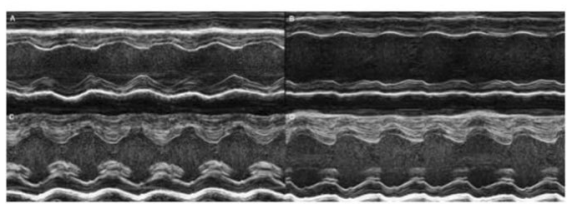

Fig. 18 (abstract P21). Panel A Changes in Fractional Shortening (FS) versus time. Experimental groups are: FVB WT, FVB OV for Melusin expression, where $n=8$ for FVB WT and $n=12$ for FVB OV. Panel $B$ Changes in Heart Rate (HR) versus time. The groups were the same in $A$. Panel C A M-mode echography of Left Ventricle (LV) at baseline of FVB WT mice. B M-mode echography of LV after $6 \mathrm{~h}$ of LPS challenge of FVB WT mice. C M-mode echography of LV at baseline of FVB OV mice. D M-mode echography of LV after $6 \mathrm{~h}$ of LPS challenge of FVB OV mice

A
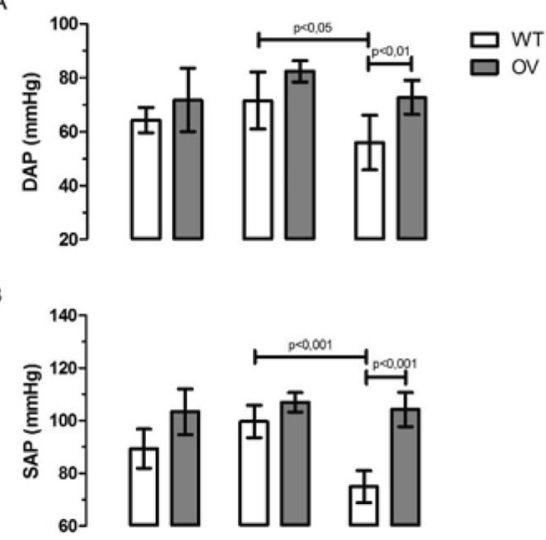

C

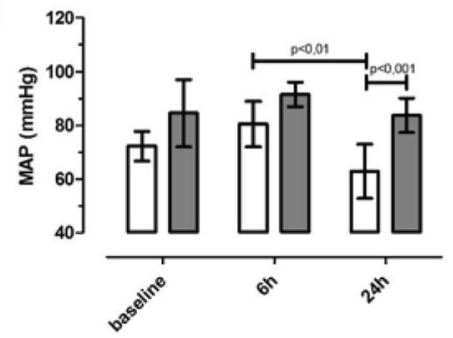

Fig. 19 (abstract P21). Panel A Changes in Diastolic Arterial Pressure (DAP) versus time. Experimental groups are: FVB WT and FVB OV for Melusin Expression, where $n=10$ for both groups. Panel $B$ Changes in Systolic Arterial Pressure (SAP) versus time. Experimental groups are the same in A. Panel C Changes in Mean Arterial Pressure (MAP) versus time. The groups are the same in A. (this figure wasn't uploaded)

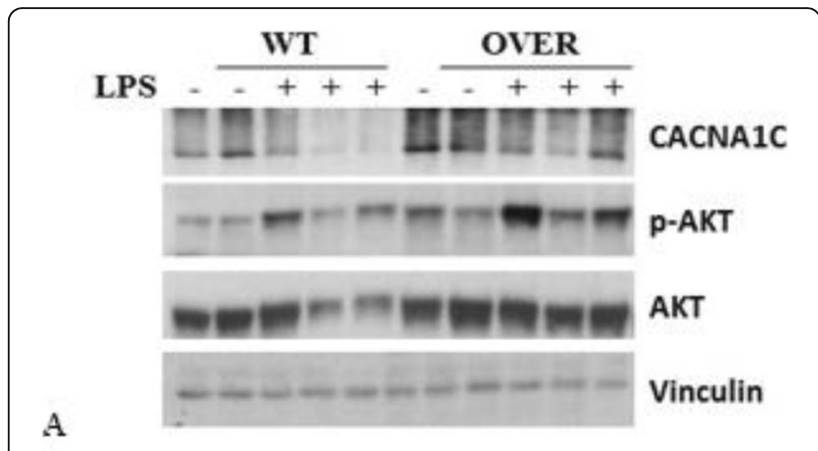

A
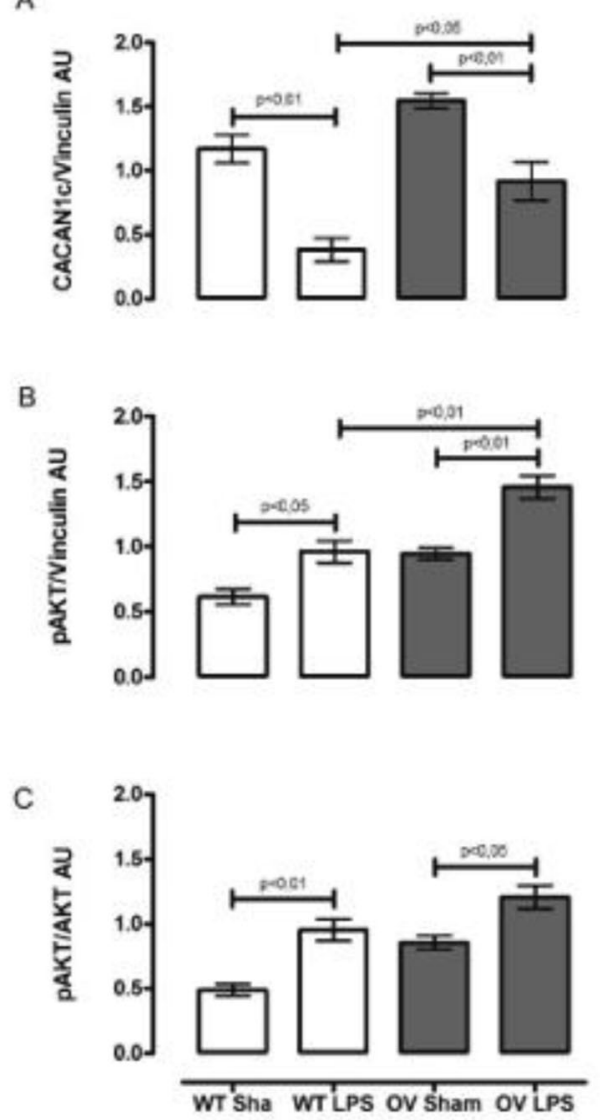

B

Fig. 20 (abstract P21). Panel A Western Blot Panel B: A Changes in CACNA1C expression versus LPS exposure. Experimental groups are: FVB WT sham and FVB OV for Melusin Expression sham, where $n=2$ for both groups, $B$ Changes in PAKT expression versus LPS exposure. Experimental groups are the same in A C Changes in PAKT/AKT expression versus LPS exposure. Experimental groups are the same in A

P22

The derivation and validation of three novel sepsis molecular subtypes to help guide precision therapies

Timothy E Sweeney ${ }^{1,2}$, Tej D Azad ${ }^{1,2}$, Michele Donato ${ }^{1,2}$, Winston A Haynes ${ }^{1,2}$, Thanneer M Perumal ${ }^{3}$, Jesús F Bermejo-Martin ${ }^{4}$, Benjamin Tang ${ }^{5-8}$,

Hector R Wong ${ }^{9,10}$, Ephraim L Tsalik ${ }^{11,12}$, Raymond J Langley ${ }^{13}$, Purvesh Khatri ${ }^{1,2}$ ${ }^{1}$ Stanford Institute for Immunity, Transplantation and Infection, Stanford University School of Medicine, Stanford, CA, US; ${ }^{2}$ Biomedical Informatics 
Research, Stanford University School of Medicine, Stanford, CA, USA; ${ }^{3}$ Sage Bionetworks, Seattle, WA, US; ${ }^{4}$ Bio•Sepsis, Hospital Clínico Universitario de Valladolid/IECSCYL, Valladolid, Spain; ${ }^{5}$ Centre for Immunology and Allergy Research, Westmead Institute for Medical Research, Sydney, Australia; ${ }^{6}$ Department of Intensive Care Medicine, Nepean Hospital, Sydney, Australia; ${ }^{7}$ Nepean Genomic Research Group, Nepean Clinical School, University of Sydney, Sydney, Australia; ${ }^{8}$ Marie Bashir Institute for Infectious Diseases and Biosecurity, Sydney, Australia; ${ }^{9}$ Division of Critical Care Medicine, Cincinnati Children's Hospital Medical Center and Cincinnati Children's Research Foundation, Cincinnati, $\mathrm{OH}$, US; ${ }^{10}$ Department of Pediatrics, University of Cincinnati College of Medicine, Cincinnati, OH, US; ${ }^{11}$ Department of Medicine, Duke University, Durham, NC, US; ${ }^{12}$ Emergency Department Service Line, Durham Veterans Affairs Health Care System, Durham, NC, USA; ${ }^{13}$ Department of Pharmacology, University of South Alabama. Mobile, $\mathrm{AL}$, US

Correspondence: Timothy Sweeney (tes17@stanford.edu) Intensive Care Medicine Experimental 2017, 5(Suppl 1):P22

\section{Background}

Sepsis is defined as a dysregulated immune response, but no immunomodulatory therapies have been shown to decrease sepsis mortality. Despite its high clinical heterogeneity, sepsis is still treated as a single disease. Defining sepsis subtypes (or 'endotypes') based on the molecular immune response may allow for a precision medicine approach to match novel therapies to those sepsis patients that can benefit. Methods

We performed a systematic search of GEO and ArrayExpress for gene expression datasets of clinical studies in sepsis, as previously described[1]. We selected studies of bacterial sepsis, sampled within 48 hours of hospital admission, across a broad range of ages, severities, and geographic locations. We used a batch effect correction algorithm[2] to pool data from 14 bacterial sepsis transcriptomic datasets from 8 different countries $(\mathrm{N}=700)$. We then used a meta-clustering algorithm[3] to find conserved sepsis subtypes across the conormalized discovery samples. We derived a classifier for application in external datasets, and then validated our clusters in 9 independent datasets from 5 different countries $(N=600)$. Clusters were evaluated for both clinical and molecular differences in both discovery and validation datasets.

Results

Meta-clustering revealed three sepsis subtypes, which, based on gene ontology analysis, we termed Inflammopathic, Adaptive, and Coagulopathic. We derived a 33-gene classifier with an overall 83\% accuracy in leave-one-out re-assignment of the discovery data. We then applied this classifier to identify the same 3 subtypes in 9 independent datasets $(\mathrm{N}=$ $600)$. Across both discovery and validation, we showed that the Inflammopathic subtype is associated with younger age, higher clinical severity and higher mortality; the Adaptive subtype is significantly associated with a lower clinical severity and lower mortality; and the Coagulopathic subtype is significantly associated with both older age and clinical coagulopathy. Further, these clusters are significantly associated with clusters derived by others in independent, single-study sepsis cohorts[4-8].

\section{Conclusions}

We identified three sepsis subtypes (Inflammopathic, Adaptive, and Coagulopathic) associated with significant difference in mortality among sepsis patients. These sepsis subtypes may assist in the development of targeted therapies that would fail if applied to all patients with sepsis[6]. The consistent presence of the sepsis subtypes across a broad range of patients from multiple studies around the world suggests their potential for use as a single framework to better define heterogeneity in patients with bacterial sepsis.

\section{Acknowledgements}

The authors wish to thank Ricardo Henao, Raquel Almansa, Eduardo Tamayo, Judith A Howrylak, Augustine Choi, Grant P Parnell, Marshall Nichols, Christopher W Woods, Geoffrey S Ginsburg, Stephen F Kingsmore, Larsson Omberg, and Lara M Mangravite for contributions to this work.

\section{References}

1. Sweeney $T E$, Khatri P: Benchmarking Sepsis Gene Expression Diagnostics Using Public Data. Crit Care Med 2017, 45(1):1-10.
2. Sweeney TE, Wong HR, Khatri P: Robust classification of bacterial and viral infections via integrated host gene expression diagnostics. Sci Transl Med 2016, 8(346):346ra391.

3. Sweeney TE, Chen AC, Gevaert O: Combined Mapping of Multiple clUsteriNg ALgorithms (COMMUNAL): A Robust Method for Selection of Cluster Number, K. Sci Rep 2015, 5:16971.

4. Wong HR, Cvijanovich N, Lin R, Allen GL, Thomas NJ, Willson DF, Freishtat RJ, Anas N, Meyer K, Checchia PA et al: Identification of pediatric septic shock subclasses based on genome-wide expression profiling. BMC Med 2009, 7:34.

5. Wong HR, Cvijanovich NZ, Allen GL, Thomas NJ, Freishtat RJ, Anas N, Meyer K, Checchia PA, Lin R, Shanley TP et al: Validation of a gene expression-based subclassification strategy for pediatric septic shock. Crit Care Med 2011, 39(11):2511-2517.

6. Wong HR, Cvijanovich NZ, Anas N, Allen GL, Thomas NJ, Bigham MT, Weiss SL, Fitzgerald J, Checchia PA, Meyer K et al: Developing a clinically feasible personalized medicine approach to pediatric septic shock. Am J Respir Crit Care Med 2015, 191 (3):309-315.

7. Davenport EE, Burnham KL, Radhakrishnan J, Humburg P, Hutton P, Mills TC, Rautanen A, Gordon AC, Garrard C, Hill AV et al: Genomic landscape of the individual host response and outcomes in sepsis: a prospective cohort study. Lancet Respir Med 2016.

8. Burnham KL, Davenport EE, Radhakrishnan J, Humburg P, Gordon AC, Hutton P, Svoren-Jabalera E, Garrard C, Hill AV, Hinds CJ et al: Shared and Distinct Aspects of the Sepsis Transcriptomic Response to Fecal Peritonitis and Pneumonia. Am J Respir Crit Care Med 2016.

\section{P23}

Histidine-rich glycoprotein as a novel prognostic biomarker for sepsis: multicenter prospective observational study

Kosuke Kuroda', Hiroshi Morimatsu', Naoya Kawanoue ', Hidenori Wake², Shuji Mori ${ }^{3}$, Masahiro Nishibori ${ }^{2}$

${ }^{1}$ Department of Anesthesiology and Resuscitology, Okayama University Graduate School of Medicine, Dentistry and Pharmaceutical Sciences, Okayama, Japan; ${ }^{2}$ Department of Pharmacology, Okayama University Graduate School of Medicine, Dentistry and Pharmaceutical Sciences, Okayama, Japan; ${ }^{3}$ Department of Pharmacology, School of Pharmacy, Shujitsu University, Okayama, Japan

Correspondence: Kosuke Kuroda (kosa_96@yahoo.co.jp)

Intensive Care Medicine Experimental 2017, 5(Suppl 1):P23

\section{Background}

There are many biomarkers and therapies for sepsis used in clinical practices, but few become the standard.

Histidine-rich glycoprotein (HRG) is a plasma glycoprotein produced in the liver, and its plasma concentration is around $80-100 \mu \mathrm{g} / \mathrm{ml}$. Our group reported that plasma HRG levels decreased in mice with sepsis, and supplemental HRG infusion significantly improved survival rate in mice model of sepsis [1]. We also showed that HRG produced strong inhibition of subsequent immunothrombosis, DIC state, hypercytokinemia, and activation of vascular endothelial cells[1]. Moreover, in our clinical study, we performed a prospective observational study of patients with systemic inflammatory response syndrome (SIRS). We found HRG levels were significantly lower in SIRS patients with infection than in those without infection, and HRG could be a novel diagnostic and prognostic biomarker for SIRS patients. In this study, we focused on the HRG levels in patients with sepsis, and assessed the differences of HRG levels between non-survivors and survivors.

Methods

This study was multicenter, prospective, observational investigation. We studied ICU patients who were newly diagnosed as sepsis. We collected blood samples within 24 hours of ICU admission, and HRG levels were determined with a quantitative enzyme-linked immunosorbent assay (ELISA). Data were expressed as median and interquartile ranges (IQR, 25th to 75th percentiles). We used Mann-Whitney U test for comparisons between the groups. We also applied Cox proportional hazard model to analyze relationship between HRG levels and mortality.

Results

Ninety-nine septic patients from 11 institutes in Japan were included. HRG levels were significantly lower in nonsurvivors $(n=16)$ than in 
survivors ( $n=83)(15.1$ [IQR, 12.7 - 16.6] vs. 30.6 [IQR, $22.1-39.6] \mu \mathrm{g} / \mathrm{ml}$; $\mathrm{p}<0.01$ ) (Fig. 21). Survival analysis revealed that HRG levels were associated with mortality (Hazard ratio $0.78 ; \mathrm{p}<0.01$ ), and the Harrell C-index (predictive power) for HRG was 0.90 . Furthermore, when patients were divided into two groups according to HRG levels using the cutoff value of $19.6 \mu \mathrm{g} / \mathrm{ml}$, lower HRG group represented significantly higher mortality $(p<0.01)$ (Fig. 22)

\section{Conclusions}

We studied 99 septic patients and demonstrated that initial HRG levels were significantly lower in non-survivors than in survivors. We also found that HRG levels at ICU admission were associated with mortality. These results suggested that HRG could be a novel prognostic biomarker for septic patients.

\section{Reference}

1. Wake H, Mori S, Liu K, Morioka Y, Teshigawara K, Sakaguchi M, Kuroda K, Gao Y, Takahashi H, Ohtsuka A, et al. Histidine-Rich Glycoprotein Prevents Septic Lethality through Regulation of Immunothrombosis and Inflammation. EBioMedicine 2016, 9: 180-194.

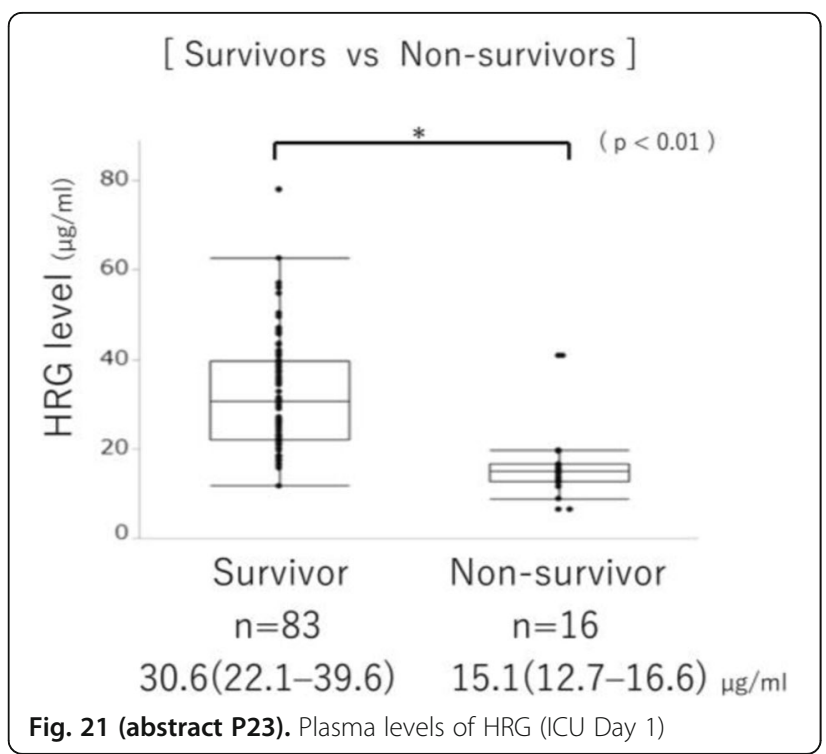

- Kaplan-Meier curve

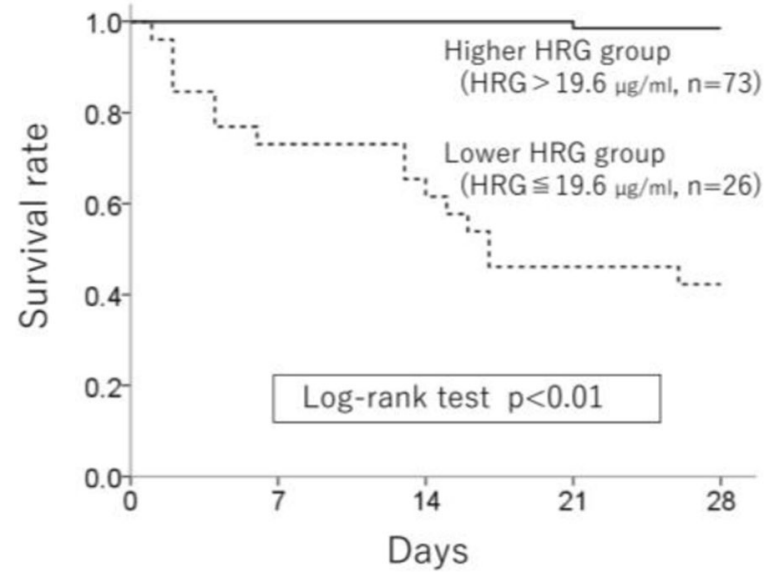

Fig. 22 (abstract P23). Survival Analysis
P24

Histine-rich glycoprotein protects vascular endothelial cells and red blood cells in sepsis

Hidenori Wake', Yuan Gao', Zhong Hui', Shangze Gao', Shuji Mori², Hiroshi Morimatsu', Masahiro Nishibori ${ }^{1}$

'Departments of Pharmacology and Anesthesiology \& Resuscitation,

Okayama University Graduate School of Medicine, Okayama, Japan;

${ }^{2}$ Shujitsu University, Faculty of Pharmaceutical Sciences, Okayama, Japan

Correspondence: Masahiro Nishibori (mbori@md.okayama-u.ac.jp)

Intensive Care Medicine Experimental 2017, 5(Suppl 1):P24

\section{Background}

Sepsis is one of the major causes of death worldwide. However, the pathogenesis of sepsis is extremely complexed and still unclear. Recently, we identified a plasma protein, histidine-rich glycoprotein (HRG), which decreased rapidly in septic mice with the cecal ligation and puncture (CLP)[1]. HRG appears to maintain a neutrophil's spherical shape with minimal microvilli on the surface. These morphological features render neutrophils quiescent with respect to the cell adhesion to vascular endothelial cells, the passage of microvasculatures, and the spontaneous release of reactive oxygen species. The supplementary treatment of CLP septic mice with human HRG dramatically improved the survival rate of CLP mice associated with the inhibition of immunothrombosis in pulmonary vasculatures, DIC state, and inflammatory responses, without interfering with the infiltration of neutrophils into peritoneal cavity. We also demonstrated the low levels of plasma HRG in septic patients in ICU. In addition to neutrophils, the vascular endothelial cells should play an important role in the progression of septic pathogenesis. Moreover, red blood cells may be involved in the enhanced pro-thrombotic state in septic condition. Therefore, in the present study, we examined the effects of HRG on vascular endothelial cells, EA.hy926, and red blood cells.

Methods

EA.hy926 cell monolayer was stimulated with LPS or TNF-alpha in the presence of PBS, human serum albumin (HSA) or HRG for indicated periods. After the stimulation, the cells were stained with by anti-CD54, anti-CD62P or anti-phosphatidylserine Ab. The cells were also stained with fluorescent phalloidin, anti-VE-cadherin or anti-bata-catenin Ab. Washed human red blood cells (RBCs) were stimulated with $\mathrm{Zn} 2+$ to observe phosphatidylserine expression and aggregation.

Results

The results showed that LPS and TNF-alpha induced actin polymerization and loss of VE- cadherin molecule between cells, associated with the up-regulation of CD54 and CD62P. These responses were inhibited by the presence of HRG (1 microM). Moreover, HRG suppressed the permeability increase of endothelial monolayer induced by LPS and TNF-alpha. HRG also protected Zn2 +-induced expression of phosphatidylserine on human RBCs and their aggregation significantly. In addition, heme-induced hemolysis was concentration-dependently inhibited by HRG through its direct binding to heme.

Conclusions

HRG protected vascular endothelial cells from strong activation by LPS and TNF-alpha. Also, HRG inhibited RBC aggregation and hemolysis, leading to the prevention of heme toxicity and excessive clotting state. These actions of HRG may contribute to anti-septic effects of systemic injection of HRG in CLP mice.

\section{Reference}

1. Wake $\mathrm{H}$, Mori S, Liu K et al: Histidine-rich glycoprotein prevents septic lethality through regulation of immunothrombosis and inflammation. EBioMedcine 2016; 9:180-194

\section{P25}

Relative and total levels of CD64 and neutrophil elastase as biomarkers for detection of sepsis

Riya Palchaudhuri, ${ }^{1,2}$, Suzanne Crowe ${ }^{1,2,3}$, Clovis Palmer ${ }^{1,2,4}$, Mary Garcia ${ }^{1}$,

Steve McGloughlin ${ }^{5}$, Shirley Vallance, ${ }^{5}$, David Anderson ${ }^{1,4}$

${ }^{1}$ Burnet Institute, Melbourne, VIC, Australia; ${ }^{2}$ Monash University,

Department of Medicine, Melbourne, Australia; ${ }^{3}$ The Alfred Hospital, 
Infectious Diseases Unit, Melbourne, Australia; ${ }^{4}$ The University of Melbourne, Department of Microbiology and Immunology, Melbourne, Australia; ${ }^{5}$ The Alfred Hospital, Intensive Care, Melbourne, Australia Correspondence: Riya Palchaudhuri (riya.palchaudhuri@burnet.edu.au) Intensive Care Medicine Experimental 2017, 5(Suppl 1):P25

\section{Background}

Improved biomarkers are required for the detection of sepsis at the point of care (POC). Upregulation of surface CD64 expression (the neutrophil CD64 index, nCD64i) has been extensively studied as a sepsis biomarker, but such assays are not amenable to use at POC. We hypothesised that simultaneous measurement of neutrophil CD64 and a neutrophil-specific protein in whole blood could yield a surrogate of the nCD64i that would be feasible for development of a simple immunoassay-based POC test. To test this hypothesis, we evaluated the relative levels of total CD64 and neutrophil elastase in whole blood of healthy controls and patients with clinically diagnosed sepsis.

Methods

We are recruiting adult ICU patients $(n=50)$ with clinically suspected sepsis (Alfred Hospital ICU, Melbourne) and healthy individuals $(n=50)$, Levels of a selected neutrophil specific marker, neutrophil elastase (NE), and neutrophil activation marker (CD64) are measured by commercial sandwich ELISA kits in detergent lysed whole blood. Samples were also tested using the Leuko64 ${ }^{T M}$ assay (Trillium Diagnostics) (surface CD64), and total (surface and intracellular) expression of CD64 was confirmed by flow cytometry staining, and microscopy.

Results

A strong correlation between total (whole blood) CD64 and NE was observed in healthy individuals $\left(n=30, R^{2}=0.7, p<0.0001\right.$, Fig. 23). Surprisingly, the total amount of CD64 reached a threshold at NE levels of only $4 \mu \mathrm{g} / \mathrm{ml}$ (Fig. 23). We established assay cutoffs (mean + 2SD) for CD64 and NE as well as a "Gating" strategy for healthy levels of CD64/ NE (Fig. 23). In sepsis patients analysed to date $(n=11)$, the Leuko64 kit was positive in $8 / 11$ patients. Conversely, total CD64 and/or NE was highly elevated in all except one myelopenic sepsis patient (10/11 positive, CD64 $p<0.0001, \mathrm{NE} p=0.0002$ relative to healthy controls), suggesting that the expected upregulation of neutrophil-CD64 during sepsis includes intracellular CD64 that is detected by ELISA, but not by the Leuko64 assay. Flow cytometry also demonstrated high levels of intracellular CD64 in selected samples that were negative by Leuko64. Conclusion

We observed saturated levels of CD64 with moderate levels of NE in controls, compared with the presence of large amounts of total CD64 (including intracellular CD64) and/or NE in whole blood of sepsis patients. Our results suggest that measurement of both total CD64 and NE levels in whole blood has promise as an improved candidate biomarker for diagnosis of sepsis compared with the detection of surface CD64 (nCD64i, Leuko64) or plasma NE.

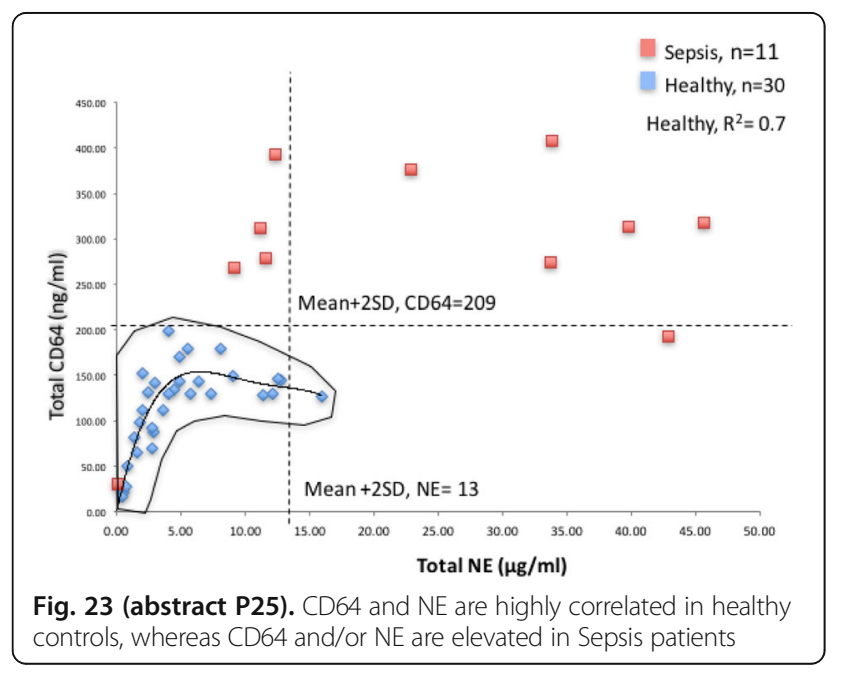

P26

Genetic determination of the dynamics of laboratory markers in septic patients

Olesya Belopolskaya ${ }^{1}$, Mariam Khadzhieva $^{1,2}$, Tamara Smelaya²,

Artem Kuzovlev², Viktor Moroz ${ }^{2}$, Arkady Goloubev², Luybov Salnikova ${ }^{1,2}$

${ }^{1}$ N.N. Vavilov Research Institute of General Genetics, Moscow, Russia;

${ }^{2}$ Federal Research and Clinical Center of Intensive Care Medicine and

Rehabilitology, Moscow, Russia

Correspondence: Artem Kuzovlev (artem_kuzovlev@mail.ru)

Intensive Care Medicine Experimental 2017, 5(Suppl 1):P26

\section{Background}

The main problem hindering the study of the genetic associations in patients with sepsis is high clinical and etiological heterogeneity. This problem can be partially solved using the approach "genotype-endophenotype- clinical phenotype".

Methods

The study involved 430 septic patients hospitalized in the intensive care units (sepsis after major trauma or extensive surgery) Biochemical analyses were performed at the 1, 3, 5, 7 and 14 days from admission. All patients were genotyped for 26 SNPs in the 21 genes of pro- (IL1b, IL6, IL8) and anti-inflammatory (IL4, IL10, IL13) cytokines, toll-like receptors (TLR2, TLR4 and TLR9), the genes of vascular remodeling (ACE, AGT, AGTR1, MTHFR, NOS3, VEGF), and the genes of the detoxification/oxidative stress response (ABCB1, AhR, CYP1A1, CAT, GCLC, NRF2). Statistical analysis was performed by logistic regression. In multivariate models we adjusted for age, sex, APACHE II score, and using of mechanical ventilation.

Results

The dynamics of changes of biochemical parameters was genetically determined by the 14th day after admission: the genotype IL6 rs 1800795 C/C (recessive model) was associated with a predisposition to increased levels of bilirubin: $P=0.0061$; difference 41.39 ( $\mathrm{mkmol} / \mathrm{L})(95 \% \mathrm{Cl}, 12.39-70.39)$, lactate dehydrogenase: $\mathrm{P}=0.0022$; difference 9.74 (U/L) $(95 \% \mathrm{Cl} 2.42-17.07)$, creatine kinase: $P=0.035$; difference 385.55 (U/L) (95\% Cl 32.29-738.80), urea: $P=0.011$; difference $276.44(\mathrm{mmol} / \mathrm{L})(95 \% \mathrm{Cl} 105.17-447.71)$. These results allowed us to reveal an additional association: patients with genotype IL6 rs $1800795 \mathrm{C} / \mathrm{C}$ had an increased chance of developing renal $(\mathrm{P}=$ $0.05, \mathrm{OR}=2.21,95 \% \mathrm{Cl} 1.00-4.78)$ and liver failure $(\mathrm{P}=0.012, \mathrm{OR}=$ $3.10,95 \% \mathrm{Cl} 1.33-7.23)$. CYP1A1 rs2606345-T/T genotype was associated with a strong excess of blood urea nitrogen $(P=0.016$, difference $-6.10,95 \% \mathrm{Cl}-10.98-1.22)$ and creatinine $\mathrm{P}=0.022$; difference $-42.03(\mathrm{mmol} / \mathrm{L})(95 \% \mathrm{Cl}-77.30--6.76)$ and kidney dysfunction among ARDS patients $(\mathrm{P}=0.00046, \mathrm{OR}=7.10,95 \% \mathrm{Cl} 2.40$ - 21.04). This genotype was associated with pneumonia and ARDS in our previous investigations (PMID: 24127120, PMID: 24068433, PMID: 24068433, PMID: 231077630).

\section{Conclusions}

Genetic studies together with clinical and laboratory data, measured in dynamics, give more information about the etiology and pathogenesis of sepsis, allowing us to study the signaling pathways associated with the onset, progression and outcome of it CYP1A1 rs2606345-T/T genotype may be considered as a potential marker of co-occurrence of ARDS and kidney injury.

\section{P27}

Heparin-binding protein improves prediction of sepsis-related acute kidney injury

Jonas Tverring ${ }^{1}$, Suvi T. Vaara ${ }^{2,3}$, Jane Fisher ${ }^{1}$, Meri Poukkanen ${ }^{4}$, Ville Pettilä2, Adam Linder

${ }^{1}$ Department of Clinical Sciences, Division of Infection Medicine, Lund University, Lund, Sweden; ${ }^{2}$ Division of Intensive Care Medicine, Department of Anesthesiology, Intensive Care and Pain Medicine, University of Helsinki and Helsinki University Hospital, Helsinki, Finland; ${ }^{3}$ Department of Intensive Care, Austin Hospital, Melbourne, Australia; ${ }^{4}$ Department of Intensive Care, Lapland Central Hospital, Rovaniemi, Finland

Correspondence: Jonas Tverring (jonas.tverring@med.lu.se)

Intensive Care Medicine Experimental 2017, 5(Suppl 1):P27 


\section{Background}

Sepsis-related acute kidney injury (AKI) accounts for major morbidity and mortality among the critically ill[1]. Heparin-binding protein (HBP) is a promising biomarker in predicting development and prognosis of severe sepsis and septic shock that has recently been proposed to be involved in the pathophysiology of AKI $[2,3]$. The objective of this study was to investigate the added predictive value of measuring plasma HBP on admission to the intensive care unit (ICU) regarding the development of septic AKI.

\section{Methods}

We included 601 patients with severe sepsis or septic shock from the prospective, observational FINNAKI study conducted in seventeen Finnish ICUs during a five-month period (1 September 2011 to 1 February 2012)[4]. The main outcome measure was development of KDIGO AKI stage 2-3 from 12 hours after admission up to 5 days. Statistical analysis for the primary endpoint included construction of a clinical risk model, area under the receiver operating curve (ROC area), category-free net reclassification index (cfNRl) and integrated discrimination improvement (IDI) with $95 \%$ confidence intervals $(95 \% \mathrm{Cl})$.

Results

Out of 511 eligible patients, 101 (20\%) reached the primary endpoint. Addition of plasma HBP to a clinical risk model significantly increased ROC area ( 0.82 versus $0.78, p=0.03)$ and risk classification scores: cfNRI $62.0 \%(95 \% \mathrm{Cl}: 40.5 \%$ to $82.4 \%)$ and IDI 0.053 (95\% Cl: 0.029 to 0.075 )

Conclusions

Plasma HBP adds predictive value to known clinical risk factors in septic AKI. Further studies are warranted to compare the predictive performance of plasma HBP to other novel AKI biomarkers.

\section{References}

1. Uchino S, Kellum JA, Bellomo R, Doig GS, Morimatsu H, Morgera S, Schetz M, Tan I, Bouman C, Macedo E et al: Acute renal failure in critically ill patients: a multinational, multicenter study. Jama 2005, 294(7):813-818.

2. Fisher J, Linder A: Heparin-binding protein: a key player in the pathophysiology of organ dysfunction in sepsis. J Intern Med 2017, 281(6):562-574

3. Fisher J, Russell JA, Bentzer P, Parsons D, Secchia S, Morgelin M, Walley KR, Boyd JH, Linder A: Heparin-Binding Protein (HBP): A Causative Marker and Potential Target for Heparin Treatment of Human Sepsis-Induced Acute Kidney Injury. Shock 2017([Epub ahead of print]).

4. Nisula S, Kaukonen KM, Vaara ST, Korhonen AM, Poukkanen M, Karlsson S, Haapio M, Inkinen O, Parviainen I, Suojaranta-Ylinen R et al: Incidence, risk factors and 90-day mortality of patients with acute kidney injury in Finnish intensive care units: the FINNAKI study. Intensive Care Med 2013, 39(3):420-428.

\section{P28}

Characterization of the safety and pharmacokinetic profile of a novel TREM-1 pathway regulating peptide (MOTREM) during a phase I, randomised, placebo controlled, first in human clinical trial

Valérie Cuvier ${ }^{1}$, Ulrike Lorch ${ }^{2}$, Stephan Witte ${ }^{1}$, Aurélie Olivier ${ }^{1}$,

Sebastian Gibot ${ }^{3,4}$, Isabel Delor ${ }^{5}$, Jean-Jacques Garaud', Marc Derive', Margarita Salcedo

${ }^{1}$ INOTREM SA, Paris, France; ${ }^{2}$ Richmond Pharmacology, St George's University of London, London, UK; ${ }^{3}$ Medical Intensive Care Unit, Hôpital Central, CHU Nancy, Nancy, France; ${ }^{4}$ INSERM U1116, Faculté de Médecine de Nancy, Université de Lorraine, Nancy, France; ${ }^{5} \mathrm{MnS}$ Modeling and Simulation, Dinant, Belgium

Correspondence: Margarita Salcedo (msm@inotrem.com)

Intensive Care Medicine Experimental 2017, 5(Suppl 1):P28

\section{Background}

MOTREM is the clinically formulated synthetic peptide (LR12) that is currently being developed for the treatment of sepsis and for the prevention of sequelae after acute myocardial infarction. Preclinical experiments have demonstrated that LR12 is able to modulate inflammatory responses and prevent their deleterious consequences in various animal models at a pharmacologically active dose of $1 \mathrm{mg} / \mathrm{kg} / \mathrm{h}[1-3]$. Its pharmacological action occurs via the Triggering Receptor Expressed on Myeloid Cells-1 (TREM-1) signalling pathway. MOTREM was safe and well tolerated in GLP toxicology studies.

Methods

Twenty-seven healthy male volunteers have been included in this clinical trial. The product was administered by continuous intravenous infusion (CIV). A single ascending dose design with 8 dose levels was used. Cohorts 1 and 2 received a 15-minutes single dose of MOTREM ( 1 and $10 \mathrm{mg}$ and one and two volunteers respectively). Then, cohorts 3 to 8 received either a 15minutes loading dose (from $0.5 \mathrm{mg} / \mathrm{kg}$ to $5 \mathrm{mg} / \mathrm{kg}$ ) followed by 7.75-hours maintenance dose (from $0.03 \mathrm{mg} / \mathrm{kg} / \mathrm{h}$ to $6 \mathrm{mg} / \mathrm{kg} / \mathrm{h}$ ) of MOTREM or a matching placebo (3:1 ratio). All volunteers were carefully monitored. Before escalation to the next dose level, safety and PK data of the previous dose level were reviewed by a safety review committee. Two follow-up visits were done at Day 8 and at Day 28. Since immune system is at rest in normal individuals and thus TREM-1 pathway is not activated, no pharmacodynamics parameters were analyzed. The main objective of this trial was then to study the safety and pharmacokinetic profile of MOTREM.

Results

No product related changes in vital signs, clinical nor laboratory parameters were observed. No product-related adverse events were re ported. The PK of MOTREM was linear; The main clearance was estimated at $463 \mathrm{~L} / \mathrm{h} / 70 \mathrm{~kg}$ which is higher than the hepatic blood flow in human (i.e., $90 \mathrm{~L} / \mathrm{h} / 70 \mathrm{~kg}$ ) and is therefore indicative of an extensive enzymatic metabolism in blood; effective half-life was calculated to be about 3 minutes

Conclusions

MOTREM was found to be safe and well tolerated up to the highest dose tested $(5 \mathrm{mg} / \mathrm{kg}$ for a 15 -minutes loading dose and $6 \mathrm{mg} / \mathrm{kg} / \mathrm{h}$ for a 7.75-hours maintenance dose). Safety and pharmacokinetics of MOTREM is currently being studied in septic shock patients in a phase lla randomised, double-blind, twostage, placebo controlled, international, multicenter clinical trial (www.clinicaltrials.gov NCT03158948).

\section{References}

[1] Derive M, Bouazza Y, Sennoun N, Marchionni S, Quigley L, Washington V, Massin F, Max JP, Ford J, Alauzet C, Levy B, McVicar DW, Gibot S.: Soluble TREM-like transcript-1 regulates leukocyte activation and controls microbial sepsis. J Immunol. 2012, 188:5585-5592.

[2] Derive M, Boufenzer A, Bouazza Y, Groubatch F, Alauzet C, Barraud D, Lozniewski A, Leroy P, Tran N, Gibot S.: Effects of a TREM-like transcript 1-derived peptide during hypodynamic septic shock in pigs. Shock 2013, 39:176-82.

[3] Derive M, Boufenzer A, Gibot S.: Attenuation of responses to endotoxin by the triggering receptor expressed on myeloid cells-1 inhibitor LR12 in nonhuman primate. Anesthesiology 2014, 120:935-42.

\section{P29}

TREM-1 is expressed by endothelial cells and plays a major role in endothelial dysfunction during experimental sepsis

Amir Boufenzer ${ }^{1}$, Lucie Jolly ${ }^{1,2}$, Kevin Carrasco ${ }^{1,2}$, Marc Derive ${ }^{1}$ Sébastien Gibot ${ }^{2}$

${ }^{1}$ INOTREM SA, Paris, France; ${ }^{2}$ INSERM U1116, Faculty of Medicine, Lorraine University, Nancy, France; ${ }^{3}$ Intensive Care Unit, Hospital Central, CHU Nancy, Nancy, France

Correspondence: Marc Derive (md@inotrem.com) Intensive Care Medicine Experimental 2017, 5(Suppl 1):P29

\section{Background}

TREM-1 (Triggering Receptor Expressed on Myeloid cells-1) is an immunoreceptor initially known to be expressed on neutrophils and monocytes/macrophages. TREM-1 plays an important role during the pathogenesis of sepsis by amplifying the inflammatory response. Modulation of TREM-1 through the administration of the LR12 peptide was linked to beneficial effects in several preclinical sepsis models [1 -3]. This study aimed to explore the mechanisms by which the clinical stage LR12 peptide prevented sepsis-induced cardiovascular dysfunction. 


\section{Methods}

Two hours after induction of fecal peritonitis, rats and mice were randomized to receive a unique $3 \mathrm{mg} / \mathrm{kg}$ intraperitoneal dose of LR12 or vehicle. Animals with laparotomia but without CLP served as controls (sham). Ex vivo vasoreactivity of mice aortic and mesenteric arteries was measured in response to phenylephrine (Phe) and acetylcholine (Ach). The activation of constitutive (Akt,eNOS) and inducible (iNOS,COX-2) endothelial pathways was assessed by WesternBlot and qRT-PCR. Survival, MAP (Mean Arterial pressure), blood lactate, intrinsic cardiac parameters and pharmacological effect of LR12 were monitored in rats. We evaluated the expression of Trem-1 in murine vessels and murine and human isolated endothelial cells by flow cytometry, qRT-PCR and confocal-microscopy.

Results

Arteries from septic mice treated with LR12 showed better vasoreactivity as compared to untreated animals. These results were confirmed in aortic and mesenteric arteries from healthy animals after in vitro stimulation with LPS or a TREM-1 agonist. We observed a reduction of the phosphorylated forms of constitutive proteins (Akt,eNOS) and in the expression of the inducible pathways (iNOS,COX-2) along with a decrease in the expression of inflammatory mediators (TNF-a, IL-6,IL-10) in arterial vessels costimulated with LR12. In rats, TREM-1 pharmacological inhibition by LR12 was associated with preservation of MAP, decreased lactate levels and an improved cardiac function as well as survival. We unexpectedly observed by qRT-PCR and confocal microscopy that TREM-1 was expressed in aorta from animals, and inducible in vivo during sepsis. These results were confirmed in isolated mice and human endothelial cells. LR12 was able to decrease the LPS-induced TREM-1, TNF-a and IL-6 expression.

\section{Conclusions}

We report here for the first time that TREM-1 is expressed and inducible in endothelial cells during sepsis. We also showed that it plays a direct role in endothelial dysfunction responsible for alteration of vasoreactivity. These results shed new lights on the mechanism of action through which the TREM- 1 inhibitory peptide LR12 displays a strong protective effect on cardiovascular function in experimental sepsis models.

\section{References}

[1] Derive M, Bouazza $Y$, Sennoun N, Marchionni S, Quigley L, Washington $V$, Massin F, Max JP, Ford J, Alauzet C, Levy B, McVicar DW, Gibot S.: Soluble TREM-like transcript-1 regulates leukocyte activation and controls microbial sepsis. J Immunol. 2012, 188:5585-5592

[2] Derive M, Boufenzer A, Bouazza Y, Groubatch F, Alauzet C, Barraud D, Lozniewski A, Leroy P, Tran N, Gibot S.: Effects of a TREM-like transcript 1-derived peptide during hypodynamic septic shock in pigs. Shock 2013, 39:176-82

[3] Derive M, Boufenzer A, Gibot S.: Attenuation of responses to endotoxin by the triggering receptor expressed on myeloid cells-1 inhibitor LR12 in nonhuman primate. Anesthesiology 2014, 120:935-42.

\section{P30}

Targeted endothelial TREM-1 deletion protects mice during septic shock

Lucie Jolly ${ }^{1,2}$, Kevin Carrasco ${ }^{1,2}$, Marc Derive ${ }^{1}$, Amir Boufenzer ${ }^{1}$,

Sébastien Gibot ${ }^{2,3}$

${ }^{1}$ INOTREM SA, France; ${ }^{2}$ INSERM U1116, Faculty of Medicine, Lorraine University, Nancy, France; ${ }^{3}$ Intensive Care Unit, Hospital Central, CHU Nancy, Nancy, France

Correspondence: Marc Derive (md@inotrem.com)

Intensive Care Medicine Experimental 2017, 5(Suppl 1):P30

\section{Background}

TREM-1 (Triggering Receptor Expressed on Myeloid cells-1) is an amplifier of the innate immune response. We recently demonstrated that TREM- 1 is expressed by endothelial cells and that the pharmacological inhibition of TREM- 1 by the clinical stage LR12 peptide displayed protective effects on the endothelium in polymicrobial sepsis. This study aims to assess the effect of a constitutive and a targeted deletion of TREM-1 on endothelial cells during experimental sepsis models.

\section{Methods}

We generated constitutive Trem-1 KO (TREM-1-/-) as well as endotheliumconditional TREM-1 KO (EndoTREM-1-/-) mice and submitted them to polymicrobial sepsis through CLP. Organs (Bone marrow (BM), spleen, lungs, aorta and mesenteric artery) and blood were harvested at different time points and analysed for cellular content (Flow cytometry), gene expression (qRT-PCR), cytokine/chemokine concentrations (ELISA), and vasoreactivity (Myograph). Survival was monitored for 1 week.

Results

During CLP-induced sepsis, TREM-1 constitutive deletion altered inflammatory cells mobilization and recruitment in lungs/spleen and favours the accumulation of reparative cells (Ly6Clow monocytes and M2 macrophages) in the lungs. This effect was even more pronounced in EndoTREM-1-/-. Consequently, the activation of many inflammatory genes was reduced in the lungs as well as the concentrations of various cyto/chemokines (MCP-1, VCAM-1, IL6). Sepsis induced a profound vascular hyporeactivity in WT mice which was prevented in TREM-1-/- mice. Interestingly, in EndoTREM-1-/-, while vasoconstriction was still slightly impaired, endothelium-dependent vasodilation remained intact. Finally, survival was improved in the Trem-1-/- group and even more in the EndoTREM-1-/- group.

Conclusions

The targeted deletion of endothelial TREM- 1 confers protection during septic shock in modulating inflammatory cells mobilization and activation and restoring vasoreactivity. The mechanism by which cellular recruitment is reduced is probably linked to a reduction of chemokines production by endothelial cells. The effect of abolition of Trem 1 on vascular tone, while impressive, deserves further investigations.

\section{P31}

Predictive value of the qSOFA in the Emergency Department

Susanne B. J. Gijsbers, Kaoutar Azijli, Tanca C. Minderhoud,

Prabath W. B. Nanayakkara

Departments of Internal Medicine and Emergency Medicine, VU University Medical Center Amsterdam, Amsterdam, the Netherlands

Correspondence: Susanne Gijsbers (s.gijsbers@vumc.nl)

Intensive Care Medicine Experimental 2017, 5(Suppl 1):P31

\section{Background}

Quick Sequential Organ Failure Assessment (qSOFA) was introduced in the 2016 to identify high-risk patients with sepsis outside the in tensive care unit (ICU). However, the value of qSOFA criteria the emergency department (ED) setting is unclear. We performed this prospective study to investigate the value of qSOFA, Systemic Inflammatory Response Syndrome (SIRS) criteria and Modified Early Warning score (MEWS) for predicting mortality and ICU admission within 30 days after presentation at the ED.

Methods

All patients visiting the ED between November 2016 and May (19th) 2017 with suspected or proven infection were enrolled in this prospective study. All components of SIRS, qSOFA and MEWS were collected at presentation at the ED. Sensitivity, specificity and the area under the receiver operating curve (AUROC) were calculated for predicting mortality and ICU admission.

Results

Of the 267 included patients, 12 (4.5\%) died within 30 days after presentation at the ED. Presence of two or more SIRS criteria predicted mortality with $66.7 \%$ sensitivity and specificity of $50.6 \%$. For qSOFA $>2$ a sensitivity of $8.3 \%$ and specificity of $94.9 \%$ was found. A MEWS $\geq 3$ predicted in-hospital mortality with $66.7 \%$ sensitivity and specificity of $58.8 \%$. Discrimination for mortality was highest for qSOFA (AUC 0.73 [95\% Cl 0.58-0.87]), followed by MEWS (AUC 0.69 [95\% Cl 0.58-0.80]) and SIRS (AUC 0.63 [0.48-0.79]). Furthermore, two or more SIRS criteria predicted ICU admission with $85.7 \%$ sensitivity and specificity of $50.6 \%$. For qSOFA $\geq 2$ a sensitivity of $14.3 \%$ and specificity of $95.0 \%$. A MEWS $\geq 3$ predicted ICU admission with $71.4 \%$ sensitivity and specificity of $58.3 \%$. ICU admission was discriminated best by SIRS (AUC 0.76 [95\% Cl 0.60-0.93]), followed by qSOFA (AUC 0.64 [95\% Cl 0.42-0.86]) and MEWS (AUC 0.61 [0.40-0.83]). However, qSOFA missed $91.7 \%$ of the deceased patients and $85.7 \%$ of patients admitted to the ICU. 
Conclusions

Among patients presenting at the ED the qSOFA criteria had high specificity, but lacked sensitivity. The value of Q-SOFA in the ED setting is in our opinion questionable.

\section{P32}

Differences of hepar and kidneys reactive histological changes in a murine model of sepsis

M. Cherkasova', S. Andreevskaya', T. Borovaya', V. Zhukhovitsky ${ }^{1,2}$

'Gamaleya Federal Research Centre for Epidemiology and Microbiology, Moscow, Russian Federation; ${ }^{2}$ Sechenov The First State Medical

University, Moscow, Russian Federation

Correspondence: M. Cherkasova (lambada9191@mail.ru)

Intensive Care Medicine Experimental 2017, 5(Suppl 1):P32

\section{Background}

Various experimental in vivo models are used in order to research in hepar and kidneys pathogenesis under sepsis and effectiveness testing of new medications[1].

Methods

The research was carried out on 27 male mice of the C57BI/6 line, weighing $18-20 \mathrm{~g}$. The animals were randomized into 5 groups of 6 animals each mice, the fifth, (control) group consisted of 3 animals. $0.5 \mathrm{ml}$ of daily broth culture of Pseudomonas aeruginosa 1840, isolated under burn disease, was injected intraperitoneally way in the dose from $1 \times$ $103 \mathrm{CFU} / \mathrm{ml}$ to $1 \times 106 \mathrm{CFU} / \mathrm{ml}$. The control group animals were injected with $0.5 \mathrm{ml}$ of sterile isotonic solution of sodium chloride. General condition of the experimental animals was monitored twice a day. At the terminal stage of the experiment the animals were euthanized, and anatomized according to a conventional technique. The extracted hepar and kidneys were fixed with $10 \%$ neutral formaline and poured into paraffin blocks. Serial sections $4 \mu \mathrm{m}$ thick were stained with haematoxylin and eosin and analyzed in a light microscope ("AxioPlus", Carl Zeiss, Germany).

Results

Hepar histological changes of all experimental animals were almost identical, although they differed in severity. Significant damage of hepatic lobule structure and hepatocyte lamina, as well as replacement of hepar pulp with amorphous eosinophil matter were obtained. The veins and capillaries of the liver showed signs of stasis of blood, veins contained fibrin clots. Other areas of hepar pulp were infiltrated with white cells, many hepatocytes showed signs of destruction. The marked changes were most pronounced in animals that received the maximum infectious dose of $P$. aeruginosa. Kidneys histological changes among the animals of all experimental groups were similar and characterized by an abrupt drop in blood filling of capillary glomerulus of cortical and juxtamedullary nephrons. Shumlyansky-Bowman capsules of the cortical nephrons were widened, while visceral layer capsule, juxtaglomerular complex and mesangium cells showed nucleus pycnosis signs. The epithelial cells of proximal convoluted tubules of cortical nephrons looked swollen with sharply enlightened cytoplasm and signs of vacuolar dystrophy. The apical parts of the epithelial cells were destroyed and the formed cellular detritus partially or completely blocked the lumens of the tubules. There was practically no destruction of the proximal tubules in the unfiltered juxtamedullary nephrons $[2,3]$.

Conclusions

Hepar and kidneys reactive histological changes in the murine model of experimental sepsis used are characterized by a number of differences based upon the histological structure specifics of their microcirculatory bloodstream.

\section{Acknowledgements}

I am extremely grateful to appreciation to my supervisors: Professor T. G. Borovaya (MD, PhD, Corresponding member of the Russian Academy of Sciences) and V. G. Zhukhovitsky (MD, PhD, SSci, head of the laboratory of indicating and ultrastructural analysis of microorganisms), for the mentoring, recommendations, forbearance, constructive advice and assistance in the course of this research.

Throughout the experiments, the animals were processed according to the ethical guidelines for the care of laboratory animals.

\section{References}

1. Deitch EA. Rodent models of intra-abdominal infection. Shock 2005, 24 Suppl 1:19-23.

2. Mayeux Ph.R., Lee A.MacMillan-Crow. Pharmacological Targets in the renal peritubular microenvironment: implications for therapy for sepsis-induced acute kidney injury//Pharmacol. Ther.-2012.-V.134(2).-P.139 - 155.

3. Langenberg C., Bellomo R., May C.N., Egi M., Wan L. Morgera S. Renal vascular resistance in sepsis//Nephron Physiol.-2006.V.104(1).-P.1-11.

P33

Operationalising a Sepsis-3 definition to determine the epidemiology of sepsis from a multi-centre cohort of UK Critical Care Units participating in the newly founded NIHR Critical Care Health Informatics Collaborative

S. K. Harris ${ }^{1,2}$, N. S. MacCallum ${ }^{1,2}$, D. A. Brealey ${ }^{1,2}$, S. Shi ${ }^{3}$, D. Perez-Suarez ${ }^{3}$,

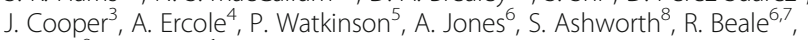
S. Brett ${ }^{9}, M$. Singer

${ }^{1}$ Bloomsbury Institute of Intensive Care Medicine, University College Hospital, London, UK; ${ }^{2}$ Critical Care, University College London Hospitals NHS Foundation Trust, London, UK; ${ }^{3}$ Research Software Development Group, Research IT Services, University College London, London, UK;

${ }^{4}$ Division of Anaesthesia, Department of Medicine, Cambridge University, Cambridge, UK; ${ }^{5}$ Critical Care Research Group (Kadoorie Centre), Nuffield Department of Clinical Neurosciences, Medical Sciences Division, Oxford University, Oxford, UK; ${ }^{6}$ Critical Care, Guy's and St. Thomas' NHS

Foundation Trust, London, UK; ${ }^{7}$ Division of Asthma, Allergy and Lung Biology, King's College, London, UK; ${ }^{8}$ Critical Care, St. Mary's Hospital, Imperial College Healthcare NHS Trust, London, UK; ${ }^{9}$ Critical Care, Hammersmith Hospital, Imperial College Healthcare NHS Trust, London, UK

Correspondence: Niall MacCallum (nsmaccallum@gmail.com) Intensive Care Medicine Experimental 2017, 5(Suppl 1):P33

\section{Background}

To report the incidence of sepsis according to the 3rd International Consensus definitions (Sepsis-3)[1] within critical care (prevalence at admission to critical care, and incidence over time), and the relative contribution of component organ dysfunction. To gain further insight into antibiotic prescribing in critical illness.

Methods

A multi-centre cohort study using a prospectively defined and validated, high-quality research database (NIHR Health Informatics Collaborative). Elective surgical admissions and those missing core physiology or mortality data were excluded. Sepsis was defined as an increase (delta) in the Sepsis-related Organ Failure Assessment (SOFA) score of two or more within a calendar day of a new antibiotic prescription (assuming no organ dysfunction prior to critical care).

Setting: Ten adult critical care units from five NHS teaching hospitals in the UK between 2014-2016.

Results

Of 17,829 critical care admissions (15,132 patients), 12,086 admissions $(10,196$ patients) were available for analysis after excluding 5,743 elective surgical admissions. We identified 14,272 episodes of life-threatening organ dysfunction (median delta-SOFA 4 [IQR 3 to 8$]$ ), predominantly around admission (point prevalence $87 \%$ $[10,447])$. The subsequent incidence was 61 per 1,000 patient days. Deteriorations at admission were largely driven by respiratory $(30 \%)$ and cardiovascular $(28 \%)$ organ dysfunction. 2,402 of all cases $(17 \%)$ were compatible with the Sepsis-3 consensus definition for shock [2]. 14,090 courses of antibiotics were prescribed to $8,502(70 \%)$ of admissions, with $53 \%(7,461)$ initiated at admission. Antibiotics were escalated during 7,110 (49.8\%) lifethreatening organ dysfunction events meeting the operational definition of Sepsis-3. Of these, 1,739 (24.5\%) met the additional criteria for septic shock [Fig. 24]. This translated into a point prevalence of sepsis on admission of 56\% (6,754 admissions), and a subsequent incidence rate of 5.8 episodes per 1,000 patient 
days. The corresponding values for septic shock were $14.2 \%$ and 0.4 per 1,000 admissions. Patients meeting this operationalised definition for Sepsis-3 had a higher ICU mortality $(16.8 \%[1,146$ deaths] versus $8.6 \%$ [453 deaths]) if cases of septic shock were included, or $11.4 \%$ [582 deaths] versus $8.6 \%$ [ 453 deaths] if shock cases were excluded. The ICU mortality for the 1,726 patients with septic shock was $32.7 \%$ (564 deaths).

Conclusions

Sepsis affects around half of all emergency admissions to critical care, with a clear progression in risk of death moving from uncomplicated deteriorations to septic deteriorations to septic shock.

\section{Acknowledgements}

This research was funded by the National Institute for Health Research Health Informatics Collaborative and supported by the National Institute for Health Research University College London Hospitals Biomedical Research Centre.

\section{References}

1. Singer $M$, Deutschman CS, Seymour CW, Shankar-Hari M, Annane $D$, Bauer M, Bellomo R, Bernard GR, Chiche JD, Coopersmith CM et al: The Third International Consensus Definitions for Sepsis and Septic Shock (Sepsis-3). JAMA 2016, 315(8):801-810.

2. Shankar-Hari M, Phillips GS, Levy ML, Seymour CW, Liu VX, Deutschman CS, Angus DC, Rubenfeld GD, Singer M, Sepsis Definitions Task F: Developing a New Definition and Assessing New Clinical Criteria for Septic Shock: For the Third International Consensus Definitions for Sepsis and Septic Shock (Sepsis-3). JAMA 2016, 315(8):775-787.

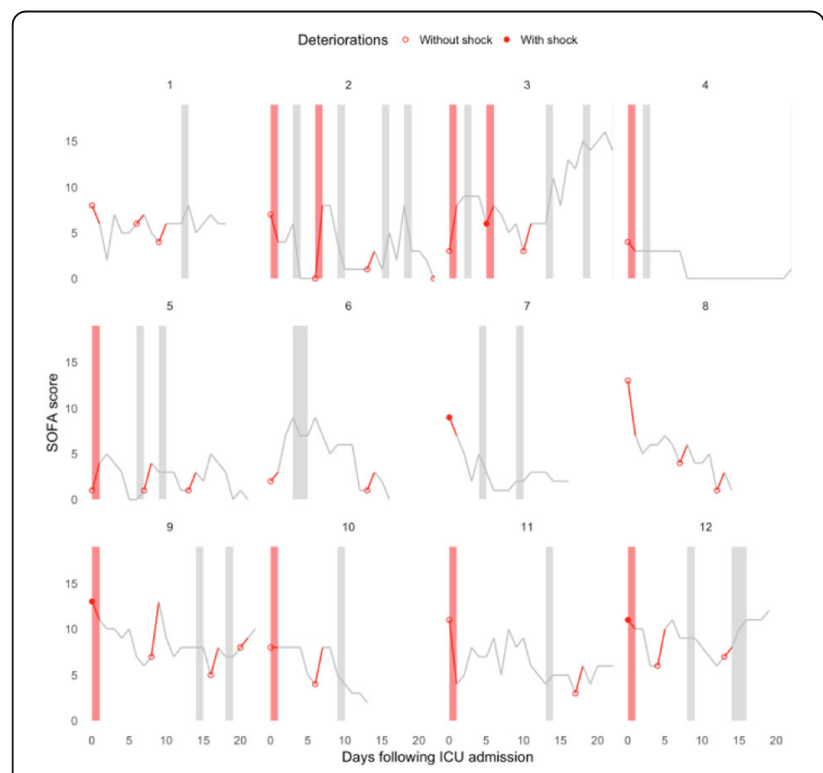

Fig. 24 (abstract P33). An example of clinical deterioration in 12 patients. Bars represent antibiotics prescriptions; red bars represent antibiotic prescriptions where SOFA $\geq 2$ and grey bars where SOFA $\leq 2$

\section{P34}

Don't blame the messenger: correcting for uncertainty in diagnosis when reporting sepsis diagnostic results

Leo C. McHugh ${ }^{1}$, Thomas D. Yager ${ }^{2}$

${ }^{1}$ Medical Science Liaison, Immunexpress, Seattle, USA; ${ }^{2}$ Director, R\&D,

Immunexpress, Seattle, USA

Correspondence: Leo McHugh (leo.m@immunexpress.com)

Intensive Care Medicine Experimental 2017, 5(Suppl 1):P34

\section{Background}

New diagnostic tests in sepsis are generally evaluated and compared in patient cohorts for which there are varying degrees of confidence in the clinical reference diagnosis for each patient. In these cohorts, even small amounts of uncertainty in the clinical reference diagnosis can have large influences on reported sensitivity and specificity that are unrelated to the true diagnostic test performance. This effect influences almost every reported sepsis diagnostic performance to date, but is rarely quantified or reported although it should be for the same reason that confidence intervals are required to contextualize results. There are currently no tools available for non-statistician researchers to address this problem. Methods

Using simulations and empirical data from sepsis trials, we show the effect of uncertainty in clinical reference diagnosis on the reported performance for diagnostic tools for sepsis. We present open-source software that automatically corrects for this effect to give more accurate performance metrics for sepsis diagnostics and can be applied retrospectively. This tool is freely available as an easy-to-use web interface that allows non-specialist researchers to upload their data to have these corrections applied. Results

We show examples of sepsis datasets for which there is sufficient uncertainty in the clinical reference diagnosis that although sensitivity and specificity are well defined, the values are statistically not meaningful. We also show the results of our software tool in normalizing performance to account for clinical uncertainty, allowing more robust comparison of molecular diagnostics over patient cohorts and between diagnostic tests. Generally, we show the usage of this tool to correct values for AUC, sensitivity, specificity, PPV and NPV in a range of sepsis datasets with varying degrees of uncertainty in the clinical reference diagnosis, for both binary diagnoses (positive/negative) and for diagnostics with a variable scale (i.e. ug/L).

Conclusions

The use of this tool allows sepsis researchers to quantify to what extent the results of a new diagnostic test are reliable in the context of uncertainty in clinical reference diagnosis. This tool can be used to identify when reference uncertainty is sufficiently high that new diagnostic performance results should be offered with caution, or not reported at all. Conversely, it may be used to qualify high confidence performance results for new tests. This tool converts the problem of uncertainty in reference diagnosis into a solution of bounding diagnostic performance estimates of new tests.

\section{Acknowledgements}

The authors wish to acknowledge Kevin Snyder, FDA statistician formerly at the Office of In Vitro Diagnostics, Division of Microbiology Devices for his contributions towards the development of this method.

\section{P35}

A combination of thalidomide and clarithromycin prevents acute lung inflammation in mice suffering from Klebsiella pneumoniae B5055 induced pneumonia

Vijay Kumar', Sanjay Chhibber ${ }^{2}$

'Department of Paediatrics and Child Care, Children's Health

Queensland Clinical unit School of Medicine, Mater Research, University

of Queensland, ST Lucia, Brisbane, Queensland, 4078, Australia;

${ }^{2}$ Department of Microbiology, Panjab University, Chandigarh, India

Correspondence: Vijay Kumar (vij_tox@yahoo.com)

Intensive Care Medicine Experimental 2017, 5(Suppl 1):P35

\section{Background}

Despite existing antibiotic therapies, acute lung injures due to acute lung infections (i.e. pneumonia) are a major cause of mortality and morbidity in both developing as well as developed countries. Agents with potent antiinflammatory as well as immunomodulatory properties are required to prevent tissue injury. These agents should be able to suppress the deleterious effects of inflammation, while leaving the protective response intact. In the present study a combination of thalidomide (THD) and clarithromycin (CLM) is used to observe their tissue protective effect in mouse model of acute lung infection induced by Klebsiella pneumoniae B5055. The two are used together as clarithromycin has both antibacterial as well as antiinflammatory properties and thalidomide has immunomodulatory property. Methods

Pneumonia was induced by intranasal instillation of Klebsiella pneumoniae B5055 (104 cfu/ml) by holding the mice in upright position without any anaesthesia. Experimental animals were divided into four groups (i.e. Group $A_{\text {; }}$ control (oral saline), Group B; clarithromycin (CLM) (30 mg/kg/day/os), Group 
C; Thalidomide (THD) ((30 mg/kg/po/day), and Group D; a combination of both thalidomide and clarithromycin (CLM + THD). At designated period of time (1, 2, 3, 5 and 7 days) mice were euthanized by cervical dislocation and lungs were harvested and homogenized in $1 \mathrm{ml}$ of sterile normal saline under sterile conditions. The lung homogenate was used for measuring bacterial load, malondialdehyde (MDA), nitric oxide (NO) and myeloperoxidase (MPO) and pro-inflammatory (i.e. IL-1a, IL-6, TNF-a) anti-inflammatory (i.e. IL-10) cytokine levels in lungs. Histopathological examination of the lungs was also carried out for marking inflammatory lung damage and neutrophil infiltration.

Results

Clarithromycin (CLM) and thalidomide (THD) also exerted the protective immunomodulatory action when given alone. However, the combination (CLM + THD) not only significantly $(p<0.05)$ decreased the bacterial load in lungs of mice infected with Klebsiella pneumoniae B5055 but also significantly ( $p<$ $0.05)$ decreased neutrophil infiltration into the lungs. The combination also significantly $(p<0.05)$ decreased myeloperoxidase $(\mathrm{MPO})$, malondialdehyde $(\mathrm{MDA})$ and nitric oxide $(\mathrm{NO})$ generation along with a significant $(p<0.05)$ reduction in the levels of pro-inflammatory cytokines (i.e. TNF-a, IL-6 and IL-1a). This combination acted in a synergistic way and resulted in reduced tissue injury as observed in histopathological sections of lung tissue.

Conclusions

Thus, thalidomide and clarithromycin together synergistically prevented acute lung inflammation occurring during acute lung infection. This combination has a great potential as an immunomodulatory approach during acute infections causing acute inflammation.

Key Words: Clarithromycin, Thalidomide, Acute lung injury (ALI), Klebsiella pneumoniae Neutrophil, Myeloperoxidase (MPO), Malondialdehyde (MDA), Nitric Oxide (NO)

\section{P36}

LC3 associated phagocytosis defects underlie phagocyte dysfunction in sepsis-induced immunosuppression Tonia Akoumianaki ${ }^{1}$, Remi Beau², Frederic Pene ${ }^{3}$, Eleni Diamantaki ${ }^{1}$ Frank L. van de Veerdonk ${ }^{4}$, Katerina Vaporidou', Mihai G. Netea ${ }^{4}$, Christos Tsatsanis ${ }^{1}$, Jean Paul Latge ${ }^{3}$, Georgios Chamilos ${ }^{1,5}$

'Department of Medicine, University of Crete, 71300, Heraklion, Crete, Greece; ${ }^{2}$ Unité des Aspergillus, Institut Pasteur, Paris, F-75015, France;

${ }^{3}$ Medical Intensive Care Unit, Cochin Hospital, Assistance Publique, Hôpitaux de Paris, Paris, France; ${ }^{4}$ Department of Internal Medicine, Radboud University Medical Center, 6500 HB Nijmegen, The Netherlands; Institute of Molecular Biology and Biotechnology, Foundation for Research and Technology, 71300, Heraklion, Crete, Greece Correspondence: Georgios Chamilos (hamilos@imbb.forth.gr) Intensive Care Medicine Experimental 2017, 5(Suppl 1):P36

\section{Background}

Despite significant advances in life support of critically ill patients, sepsis remains a major cause of mortality worldwide[1]. Currently, most sepsis patients survive the initial sepsis episode to enter a prolonged state of immune deactivation, termed sepsis-induced immunosuppression, which accounts for treatment failure and death due to secondary infection(s)[2]. While the clinical impact of sepsis immunosuppression is well recognized, the underlying mechanisms remain unknown. Evermore, the failure of over 100 clinical trials on sepsis immunotherapy has been largely attributed to the lack of reliable biological measures that discriminate patients with immune deactivation[2]. We recently demonstrated the major role of a specialized autophagy pathway termed LC3 associated phagocytosis (LAP) in immunity against the major human fungal pathogen Aspergillus fumigatus[3, 4]. Because A. fumigatus is an emerging pathogen in ICU patients recovering from sepsis, we explored whether defective LAP is implicated in sepsis immunosuppression. Methods

In a prospective study we evaluated phagocyte function in adult patients ( $>18$ years old) admitted in the ICU of two University Hospitals with community-acquired septic shock. Monocytes isolated during early (Day 1 ) and late (Day 7) phase of sepsis were stimulated with A. fumigatus bioparticles and phagosome biogenesis was assessed by confocal imaging. Phagosome studies were also performed in a physiologically relevant model of peritonitis in mice with different degree of sepsis severity.

Results

We identified a distinct group of patients $(12 / 30,40 \%)$ who developed LAP blockade in monocytes upon sepsis recovery (Fig. 25). LAP blockade was associated with broad phagosome biogenesis defects and impaired phagolysosomal fusion, TLR hypo-responsiveness, increased severity of illness (higher APACHE II score) and significant increase in risk for development of secondary infections $(P=0.04)$ (Fig. 25). Experiments in the CLP model of polymicrobial sepsis mirrored the findings of human studies by demonstrating selective inhibition of LAP in macrophages of mice recovering from severe sepsis, without evidence of LAP defect in macrophages obtained from mild sepsis or sham control mice (Fig. 26). Importantly, studies in macrophages from cytokine KO mice demonstrated a causal relationship between deregulated cytokine signaling pathways and LAP defects (Fig. 27).

Conclusions

Our study provides a novel mechanistic link between defects in molecular pathways regulating phagosome biogenesis and development of sepsis immunosuppression. Delineating the molecular mechanism of sepsis-induced LAP blockade will pave the way for personalized medicine and novel immunomodulatory therapies in sepsis.

\section{Acknowledgements}

This work was supported by grants from the Greek State Scholarship Foundation (I.K.Y.), the Hellenic General Secretariat for Research and Technology-Excellence program (ARISTEIA), and a Research Grant from Institut Mérieux.

\section{References}

1. Hotchkiss, R.S. et al. Sepsis-induced immunosuppression: from cellular dysfunctions to immunotherapy. Nat Rev Immunol. 2013 13(12): p. 862-74.

2. Benjamim, C.F., et al., Reversal of long-term sepsis-induced immunosuppression by dendritic cells. Blood, 2005. 105(9): p. 3588-95.

3. Kyrmizi, l., et al., Corticosteroids block autophagy protein recruitment in Aspergillus fumigatus phagosomes via targeting dectin-1/Syk kinase signaling. J Immunol. 2013 191(3): p. 1287-99.

4. Akoumianaki T, et al. Aspergillus Cell Wall Melanin Blocks LC3-Associated Phagocytosis to Promote Pathogenicity. Cell Host Microbe. 2016 19:79-90.

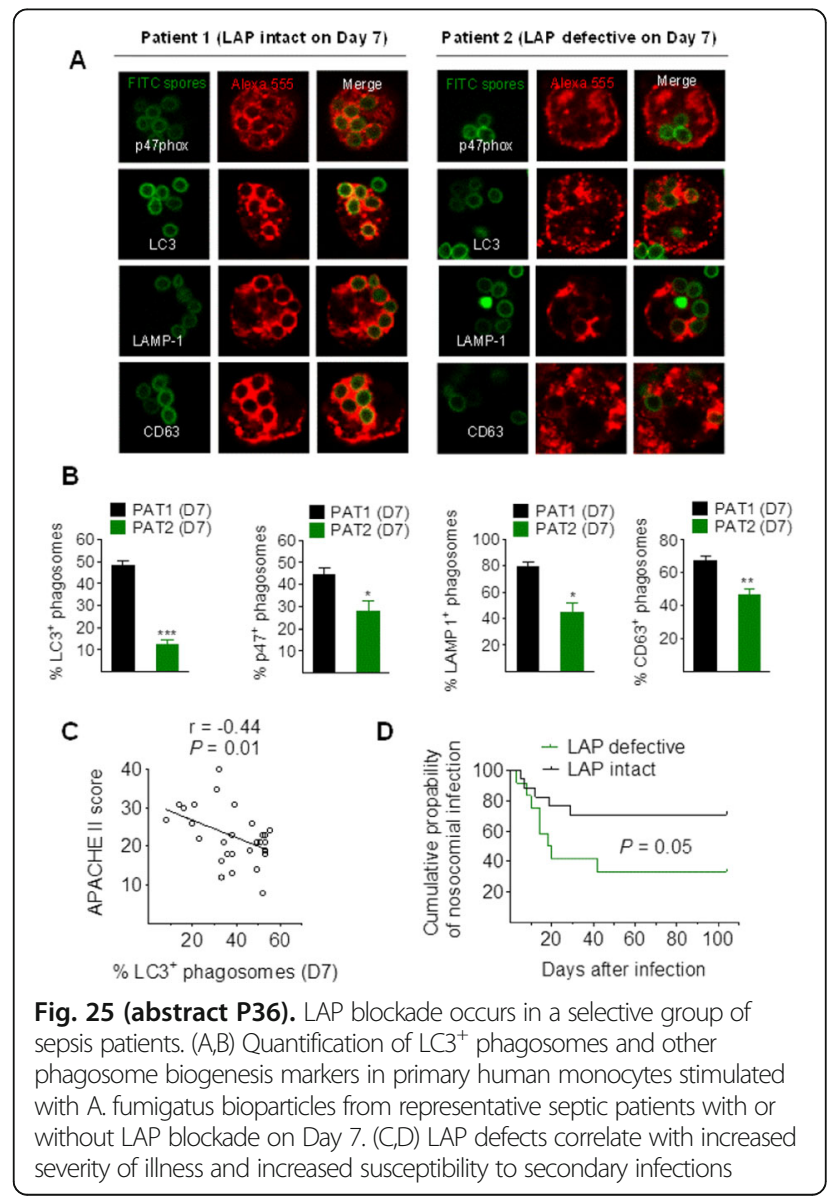




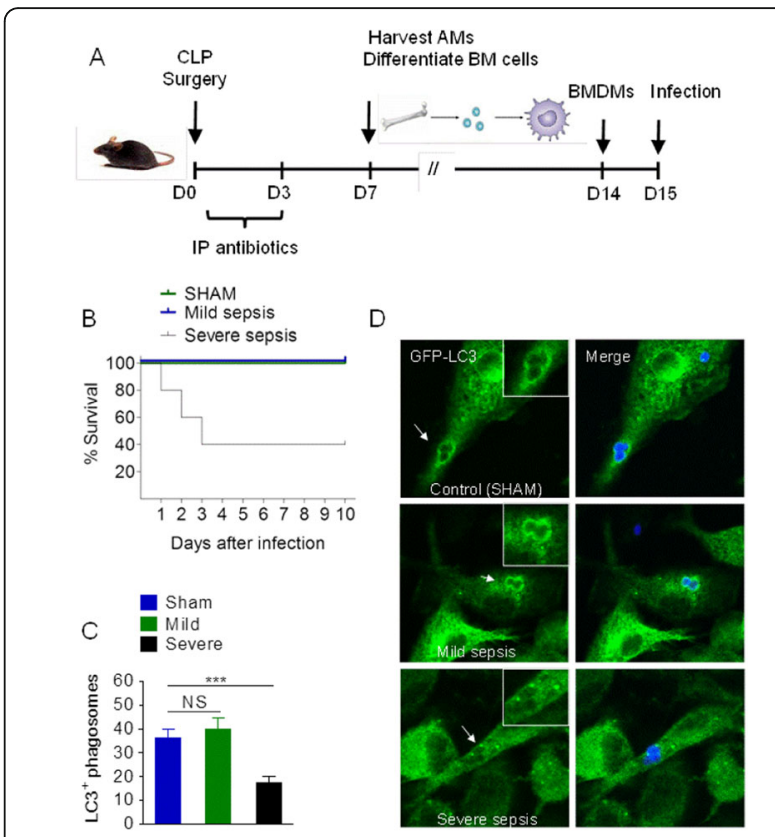

Fig. 26 (abstract P36). LAP defects selectively occur in BMDMs of mice recovering from severe sepsis. $(A, B)$ Murine model of sepsis-induced immunosuppression and outline of macrophage studies. (C,D) Selective inhibition of LAP in BMDMs of GFP-LC3 mice revovering from severe sepsis. Data on quantification of $\mathrm{LC}^{+}$phagosomes in BMDMs stimulated Aspergillus bio-particles (shown in blue) and representative confocal imaging

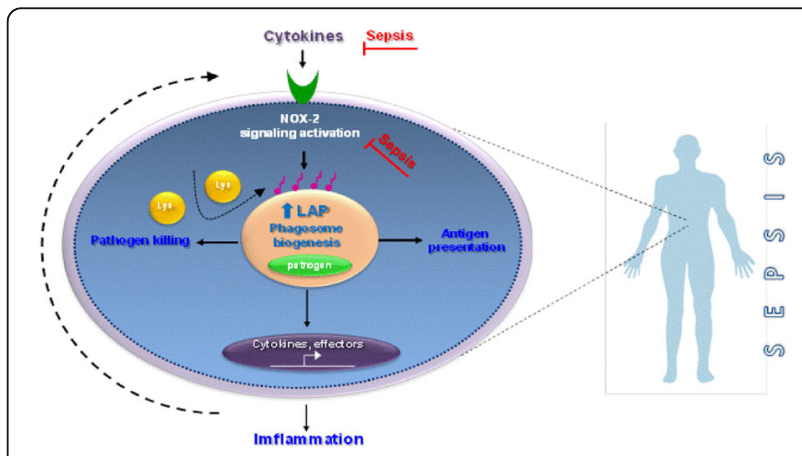

Fig. 27 (abstract P36). Proposed model on the mechanism of LAP defects and phagocyte dysfunction in patients with sepsis immunosuppression. Defective cytokine signaling upstream of $\mathrm{NADPH}$ oxidase inhibits activation of LC3 associated phagocytosis (LAP) and results in multiple levels of phagocyte dysfunction, which is the cellular hallmark of immune deactivation observed in patients with sepsis

\section{P37}

\section{Withdrawn}

\section{P38}

Low endocan levels at ICU admission are predictive of acute respiratory distress syndrome in severe sepsis and septic shock Alexandre Gaudet 1,2,3,4, , Erika Parmentier ${ }^{1,2,3,4,5}$, Sylvain Dubucquoi ${ }^{6}$, Philippe Lassalle ${ }^{1,2,3,5}$, Nathalie De Freitas Caires ${ }^{1,2,3,5,7}$, Daniel Mathieu ${ }^{1,2,3,4,5}$
${ }^{1}$ Univ. Lille, U1019 - UMR 8204 - CIIL - Center for Infection and Immunity of Lille, F-59000, Lille, France; ${ }^{2}$ CNRS, UMR 8204, F-59000, Lille, France; ${ }^{3}$ INSERM, U1019, F-59000, Lille, France; ${ }^{4} \mathrm{CHU}$ Lille, Pôle de Réanimation, Hôpital Roger Salengro, F-59000, Lille, France; ${ }^{5}$ nstitut Pasteur de Lille, F-59000, Lille, France; ${ }^{6} \mathrm{CHU}$ Lille, Institut d'Immunologie, Centre de Biologie Pathologie Génétique, F-59000, Lille, France;

${ }^{7}$ Lunginnov, 1 Rue du Pr Calmette, F-59000, Lille, France

Correspondence: Alexandre Gaudet (alexandre.gaudet@yahoo.fr) Intensive Care Medicine Experimental 2017, 5(Suppl 1):P38

\section{Background}

Endocan is a circulating proteoglycan dosed at high blood levels during severe sepsis, with a likely lung anti-inflammatory role[1-3]. Data from a pilot study suggest that paradoxically low blood levels of endocan at the acute phase of severe sepsis could predict Acute Respiratory Distress Syndrome (ARDS) within $72 \mathrm{~h}[4]$. The main objective of this study was to confirm the prognostic value of low blood levels of endocan at the acute phase of severe sepsis to predict ARDS.

Methods

We constituted a validation cohort in a prospective single-center observational study including patients admitted for severe sepsis in the Intensive Care Unit (ICU) of a French University Hospital, between October 2014 and March 2016. Blood endocan was measured at inclusion. Criteria for ARDS were evaluated at $12 \mathrm{~h}, 24 \mathrm{~h}, 48 \mathrm{~h}$ and $72 \mathrm{~h}$ following inclusion. The primary outcome was a lower endocan blood level at inclusion in patients with ARDS at $72 \mathrm{~h}$.

Results

100 patients admitted in ICU for severe sepsis were included. 31 patients met criteria for ARDS at $72 \mathrm{~h}$.

Endocan blood values at inclusion were significantly lower in patients who met the criteria of ARDS at $72 \mathrm{~h}(p=0.01)$. For each elevation of $1 \mathrm{ng} / \mathrm{mL}$ of endocan, the adjusted $O R$ for presence of ARDS at $72 \mathrm{~h}$ was of $0.93(95 \% \mathrm{Cl} 0.86-0.998 ; \mathrm{p}=0.045)$. In our cohort, an endocan value $<2.54 \mathrm{ng} / \mathrm{mL}$ predicted ARDS at $72 \mathrm{~h}$ with a positive predictive value of $0.75(\mathrm{Sp}=0.97(95 \% \mathrm{Cl}$ $0.9-1))$.

Conclusions

In a validation cohort of severe septic patients, we confirmed that low blood levels of endocan at ICU admission are predictive of ARDS at $72 \mathrm{~h}$.

\section{References}

1. Lassalle P, Molet S, Janin A, Heyden JV, Tavernier J, Fiers W, et al. ESM-1 is a novel human endothelial cell-specific molecule expressed in lung and regulated by cytokines. J Biol Chem. 23 août 1996;271(34):20458-64.

2. Béchard D, Scherpereel A, Hammad H, Gentina T, Tsicopoulos A Aumercier $\mathrm{M}$, et al. Human endothelial-cell specific molecule-1 binds directly to the integrin CD11a/CD18 (LFA-1) and blocks binding to intercellular adhesion molecule-1. J Immunol Baltim Md 1950. 15 sept 2001;167(6):3099-106.

3. Scherpereel A, Depontieu F, Grigoriu B, Cavestri B, Tsicopoulos A, Gentina T, et al. Endocan, a new endothelial marker in human sepsis. Crit Care Med. févr 2006:34(2):532-7.

4. Palud A, Parmentier-Decrucq E, Pastre J, De Freitas Caires N, Lassalle P, Mathieu D. Evaluation of endothelial biomarkers as predictors of organ failures in septic shock patients. Cytokine. juin 2015;73(2):213-8.

\section{P39}

Endocan and its major catabolite $\mathrm{p} 14$ regulate leukocyte diapedesis through control of the LFA-1/ICAM-1 interaction Alexandre Gaudet $1,2,3,4,5$, Lucie Portier 1,2,3,4,5, Anne Tsicopoulos ${ }^{1,2,3,5}$, Daniel Mathieu ${ }^{1,2,3,4,5}$, Philippe Lassalle ${ }^{1,2,3,5}$, Nathalie De Freitas Caires ${ }^{1,2,3,5,6}$ 'Univ. Lille, U1019 - UMR 8204 - CIIL - Center for Infection and Immunity of Lille, F-59000, Lille, France; ${ }^{2}$ CNRS, UMR 8204, F-59000, Lille, France; ${ }^{3}$ INSERM, U1019, F-59000, Lille, France; ${ }^{4} \mathrm{CHU}$ Lille, Pôle de Réanimation, Hôpital Roger Salengro, F-59000, Lille, France; ${ }^{5}$ nstitut Pasteur de Lille, F-59000, Lille, France; ' ${ }^{6}$ Lunginnov, 1 rue du Pr Calmette, F-59000, Lille, France

Correspondence: Alexandre Gaudet (alexandre.gaudet@yahoo.fr) Intensive Care Medicine Experimental 2017, 5(Suppl 1):P39 


\section{Background}

Acute respiratory Distress Syndrome (ARDS) is a severe respiratory failure, occurring in up to $20 \%$ of patients admitted in ICU with severe sepsis[1,2]. Despite years of research, the prognostic of ARDS remains associated with high levels of mortality, reaching up to $50 \%$ in most severe forms[3]. Dysregulated leukocyte diapedesis is a major mechanism leading to the constitution of ARDS[4]. Endocan is a $50 \mathrm{kDa}$ circulating proteoglycan, secreted by lung endothelial cells under pro-inflammatory conditions, which might inhibit leukocyte diapedesis by blocking the interaction between the integrin LFA-1 and its endothelial ligand ICAM-1[5]. On a clinical point of view, paradoxically low blood levels of endocan during acute systemic inflammatory states are associated with the occurence of ARDS[6,7]. This could be explained by a lack of lung anti-inflammatory activity in patients exhibiting low blood levels of endocan. At the opposite, this association could be explained by a potential pro-inflammatory role of endocan's major catabolite, p14, which has been dosed in the plasma of septic patients[8]. The objective of this study was to evaluate the respective roles of endocan and of its major catabolite p14 in the regulation of leukocyte diapedesis.

Methods

We explored in vitro the roles of endocan and p14 in the control of human T cell transendothelial migration, as well as in ICAM-1 mediated migration and ICAM-1 dependant adhesion. We also evaluated at the molecular scale the impact of p14's binding to LFA-1 on the endocan/LFA-1 interaction. Finally, we explored the effect of p14 on the ICAM-1/LFA-1 interaction.

Results

In our in vitro study, endocan inhibited human T cell transendothelial migration, as well as ICAM-1 dependant migration and ICAM-1 mediated adhesion. At the opposite, p14 had the ability to reverse endocan's effects on T cell transendothelial migration, ICAM-1 dependant migration and ICAM-1 mediated adhesion. Our molecular study revealed that p14 could bind to the integrin LFA-1 and inhibit the endocan/LFA-1 interaction. Finally, we could not observe any effect of p14 on the interaction between LFA-1 and ICAM-1.

Conclusions

The results of our study highlight the ICAM-1 dependant antimigratory effect of endocan on human leukocytes. Our study also reveals the potential role of p14 as a biological competitor of endocan, consisting in the restoration of leukocyte diapedesis through restoration of the LFA-1/ICAM-1 interaction.

\section{References}

1. Gajic O, Dabbagh O, Park PK, Adesanya A, Chang SY, Hou P, et al. Early identification of patients at risk of acute lung injury: evaluation of lung injury prediction score in a multicenter cohort study. Am J Respir Crit Care Med. 15 févr 2011;183(4):462-70.

2. Mikkelsen ME, Shah CV, Meyer NJ, Gaieski DF, Lyon S, Miltiades AN, et al. The epidemiology of acute respiratory distress syndrome in patients presenting to the emergency department with severe sepsis. Shock Augusta Ga. nov 2013;40(5):375-81.

3. ARDS Definition Task Force, Ranieri VM, Rubenfeld GD, Thompson BT, Ferguson ND, Caldwell $E$, et al. Acute respiratory distress syndrome: the Berlin Definition. JAMA. 20 juin 2012;307(23):2526-33.

4. Ware LB, Matthay MA. The acute respiratory distress syndrome. N Engl J Med. 4 mai 2000;342(18):1334-49.

5. Béchard D, Scherpereel A, Hammad H, Gentina T, Tsicopoulos A, Aumercier $M$, et al. Human endothelial-cell specific molecule-1 binds directly to the integrin CD11a/CD18 (LFA-1) and blocks binding to intercellular adhesion molecule-1. J Immunol Baltim Md 1950. 15 sept 2001;167(6):3099-106.

6. Palud A, Parmentier-Decruca E, Pastre J, De Freitas Caires N, Lassalle P, Mathieu D. Evaluation of endothelial biomarkers as predictors of organ failures in septic shock patients. Cytokine. juin 2015;73(2):213-8.

7. Mikkelsen ME, Shah CV, Scherpereel A, Lanken PN, Lassalle P, Bellamy SL, et al. Lower serum endocan levels are associated with the development of acute lung injury after major trauma. J Crit Care. oct 2012;27(5):522.e11-17.

8. De Freitas Caires N, Legendre B, Parmentier E, Scherpereel A, Tsicopoulos A, Mathieu $D$, et al. Identification of a $14 \mathrm{kDa}$ endocan fragment generated by cathepsin $\mathrm{G}$, a novel circulating biomarker in patients with sepsis. J Pharm Biomed Anal. 5 mai 2013;78-79:45-51.
P40

Inference of the etiology of bloodstream infections (sepsis) based on procalcitonin value

C. Peronace ${ }^{1}$, A. Quirino ${ }^{1}$, A. Giancotti ${ }^{1}$, L. Galati ${ }^{1}$, L. Gallo', P. Settembre ${ }^{1}$ G. Cortese', P. Morelli', E. Garofalo², G.S. Barreca', A.G. Lamberti1, G. Matera $^{1}$, P. Navalesi ${ }^{2}$, M.C. Liberto ${ }^{1}$, A. Focà ${ }^{1}$

${ }^{1}$ Institute of Microbiology, Department of Health Sciences, University "Magna Graecia" of Catanzaro, Catanzaro, Italy; ${ }^{2}$ Intensive Care Unit, Department of

Health Sciences, "Magna Graecia" University of Catanzaro, Catanzaro, Italy

Correspondence: C. Peronace (cinziaperonace@hotmail.it)

Intensive Care Medicine Experimental 2017, 5(Suppl 1):P40

\section{Background}

During previous investigations, we assessed the diagnostic and prognostic role of several sepsis markers including C-reactive protein (CRP), sCD25, IL-10, procalcitonin (PCT), IL- 35 and presepsin[1-3]. Furthermore, our group mechanistically evaluated PCT in in vitro models of sepsis, thus demonstrating the LPS binding and neutralizing activity of PCT[4]. In this study, we were aiming to estimate which group of microorganisms were causing sepsis, on the basis of PCT serum levels, in order to contribute to a timely and appropriate management of this often fatal syndrome

Methods

This retrospective observational clinical study was conducted using clinical and routine laboratory data collected from June 2016 to June 2017. Blood cultures (BC) and serum samples were serially collected from each patient with suspected sepsis, defined according to the most recent guidelines[5]. Only the first sample, obtained during the first septic episode, was evaluated for each patient. Samples for blood cultures were obtained under aseptic conditions in all patients, incubated and monitored for seven days by BacT/Alert 3D system (bioMérieux, France). The isolated microorganisms and antibiotic susceptibility were assessed by standard clinical microbiology methods or automated systems. Serum PCT levels were measured by VIDAS B.R.A.H.M.S. PCT (bioMérieux, France), in the samples of enrolled patients, as well as in samples of a group of non-bacteremic controls. Statistics were obtained using ANOVA, as well as PLSD post hoc test. Receiver-operating characteristic (ROC) analysis was also performed. A $p<0.05$ was considered statistically significant.

Results

From our data, we can estimate different levels of PCT in different patients stratified by the microorganism isolated in their blood cultures. More specifically the PCT concentration in patients infected by an Enterobacteria organism was very significantly $(p=0.0001)$ different from patients associated with Gram-positive sepsis. Also, we found that levels of PCT in Nonfermentative Gram-negatives were significantly lower $(p=0.014)$ versus Enterobacteria-caused sepsis. Similarly, PCT showed a significantly lower $(p=0.031)$ concentration in fungal sepsis in comparison to sepsis caused by Enterobacterial organisms (Fig. 28). Conclusions

Previously we demonstrated that presepsin levels are differentially affected by different type of blood culture isolates, whose Enterobacteria were associated with very high presepsin levels. Similarly, in the present study, significantly higher PCT concentrations were found in sepsis cases, where blood culture isolates belonged to Enterobacteria.

\section{References}

1. Matera G, Puccio R, Giancotti A, Quirino A, Pulicari MC, Zicca E, Caroleo S, Renzulli A, Liberto MC, Focà A. Impact of interleukin-10, soluble CD25 and interferon- $\gamma$ on the prognosis and early diagnosis of bacteremic systemic inflammatory response syndrome: a prospective observational study. Crit Care. 2013, 17(2): R64

2. Peronace C, Matera G, Galati L, Giancotti A, Barreca GS, Quirino A, Liberto MC, Focà A. IL-35 and soluble CD14 subtype might be useful prognostic markers in critical septic patients. Critical Care 2016, 20(Suppl 3):357. P24

3. Matera G, Quirino A, Peronace C, Settembre P, Marano V, Loria MT, Marascio N, Galati L, Barreca GS, Giancotti A, Amantea B, Liberto MC, Focà A. Soluble CD14 Subtype-A New Biomarker in Predicting the Outcome of Critically III Septic Patients. Am J Med Sci. 2017, 353(6):543-551

4. Matera G, Quirino A, Giancotti A, Pulicari MC, Rametti L, Rodríguez ML, Liberto MC, Focà A. ProcalcitoninneutralizesbacterialLPS and reduces 
LPS-induced cytokine release in human peripheral blood mononuclear cells. BMC Microbiol. 2012, 12:68

5. Singer $M$, Deutschman CS, Seymour CW, Shankar-Hari M, Annane $D$, Bauer M, Bellomo R, Bernard GR, Chiche JD, Coopersmith CM, Hotchkiss RS, Levy MM, Marshall JC, Martin GS, Opal SM, Rubenfeld GD, van der Poll T, Vincent JL, Angus DC. The Third International Consensus Definitions for Sepsis and Septic Shock (Sepsis-3). JAMA. 2016, 315(8):801-10

$*_{p}<0.05$ vs. Gram-negative enterobacterials following ANOVA plus PLSD test

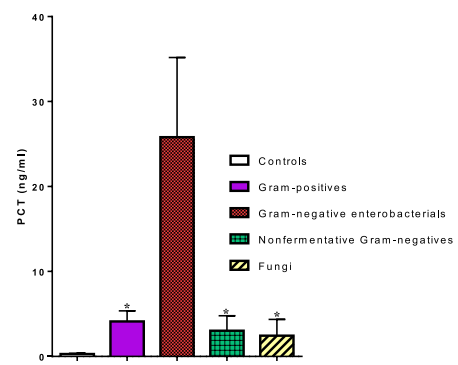

Fig. 28 (abstract P40). Levels of PCT in serum samples of Controls (non-bacteremic controls) and septic patients, whose blood cultures were found positive for Gram-positives, Gram-negative enterobacterials, Nonfermentative Gram-negatives and Fungi. * $p<0.05$ vs. Gram-negative enterobacterials following ANOVA plus PLSD test

\section{P41}

Evaluation of an automated microscopy-based multiplex FISH assay for bacteria and yeasts identification in positive blood cultures A. Focà', C. Peronace ${ }^{1}$, A. Quirino ${ }^{1}$, L. Galati ${ }^{1}$, G.S. Barreca', L. Gallo ${ }^{1}$ P. Morelli ${ }^{1}$, G. Cortese', M.C. Reale ${ }^{1}$, P. Settembre', I. Addolorato ${ }^{2}$, G. Matera', M.C. Liberto', P. Minchella ${ }^{2}$

${ }^{1}$ Institute of Microbiology, Department of Health Sciences, University "Magna Graecia" of Catanzaro, Catanzaro, Italy; ${ }^{2}$ Unit of Mirobiology and Virology, "Pugliese-Ciaccio" Hospital, Catanzaro, Italy

Correspondence: A. Focà (afoca@unicz.it)

Intensive Care Medicine Experimental 2017, 5(Suppl 1):P41

\section{Background}

Rapid identification (ID) and antimicrobial susceptibility testing (AST) are crucial to start appropriate antibiotic stewardship particularly in septic patients. The identification of organisms and drug resistance phenotype characterization from positive blood cultures generally takes several days. However, recently developed rapid diagnostic methods offer the potential for organism identification within only a few hours of blood culture positivity. The aim of this study was to evaluate the performance of an automated microscopy-based multiplex FISH assay to rapidly identify organisms directly from positive blood cultures and perform drug resistance phenotype. Methods

Sixty two positive blood cultures, obtained from critical patients admitted to Pugliese-Ciaccio Hospital of Catanzaro (Italy), were analyzed by automated Accelerate Pheno ${ }^{\mathrm{TM}}$ System (Accelerate Diagnostics Inc.) and in parallel compared to conventional methods by Vitek2 or automated mass spectrometry microbial identification system that uses MALDI-TOF technology (bioMérieux ${ }^{\circledast}$ ). The Accelerate Pheno ${ }^{\mathrm{TM}}$ System identified microbial cells using a broad panel of fluorescence in situ hybridization (FISH) probes in parallel channels. Following a one hour pre-growth and automated dilution step, remaining sample was automatically split and immobilized in separate flowcells for identification phase of the assay (90 min) and AST testing $(7 \mathrm{hr})$. Single concentrations of each appropriate antibiotic in cation-adjusted Muller-Hinton broth with $0.94 \%$ agar were introduced into each flowcell. Automated microscopy captured time-lapse images of each flowcell which were used by the computer software to analyse growth rates and other characteristic features of each individual immobilized progenitor bacterial cell during growth. An algorithm converted bacterial growth or inhibition for each tested antibiotic into a minimum inhibitory concentration (MIC) value. No matching data were also evaluated by molecular method (FilmArray, bioMérieux ${ }^{\circledast}$ ).
Results

Of the overall 62 positive blood cultures tested, six specimens were not included in the analysis due to cassette error of the instrument. Data obtained by the Accelerate Pheno ${ }^{\mathrm{TM}}$ System was confirmed in $82.1 \%$ (46/56 cases) when compared to Vitek2 method or automated mass spectrometry microbial identification system while in 17.9\% (10/56 cases) were discordant. The method was not capable of consistently identifying polymicrobial cultures in their entirety recognizing, in one blood culture, Klebsiella pneumoniae but not Candida parapsilosis since not included in the panel, or completely failing. Concerning antibiotic susceptibility testing, the Accelerate Pheno ${ }^{\mathrm{TM}}$ System showed a concordance of $86.8 \%$ when compared to Vitek2 method.

Conclusions

The data of this study demonstrated that the Accelerate Pheno ${ }^{\mathrm{TM}}$ System appears a rapid and valid method for identification and antibiotic susceptibility assessment directly from positive blood cultures. Rapid AST results provide information necessary for optimization of antimicrobial stewardship.

P42

Quantification of LCN2/HLA-DRA response by next generation-PCR to improve sepsis diagnosis in surgical patients

Raquel Almansa', Marta Martín-Fernández', Alicia Ortega',

Eduardo Tamayo ${ }^{2}$, César Aldecoa ${ }^{3}$, Ana Ávila-Alonso', Diana Benavides ${ }^{4}$,

María Heredia-Rodríguez², Silvia Martín ${ }^{3}$, Jesús F Bermejo-Martín ${ }^{1}$

${ }^{1}$ Group for Biomedical Research in Sepsis (Bio-Sepsis), Hospital Clínico

Universitario de Valladolid/IECSCYL, Valladolid, Spain; ${ }^{2}$ Anesthesiology and Reanimation Service, Hospital Clínico Universitario de Valladolid, Valladolid, Spain; ${ }^{3}$ Anesthesiology and Reanimation Service, Hospital Universitario Río Hortega, Valladolid, Spain; ${ }^{4}$ General Surgery Service, Hospital Clínico Universitario de Valladolid, Valladolid, Spain

Correspondence: Raquel Almansa (ralmansa@saludcastillayleon.es) Intensive Care Medicine Experimental 2017, 5(Suppl 1):P42

\section{Background}

Sepsis has been classically defined as the exuberant, harmful, proinflammatory response to infection. This concept is changing [1] and the presence of a dysregulated host response to infection causing life-threatening organ dysfunction is now considered a central event in the pathogenesis of the disease[2]. Quantification of the expression of immunological genes participating in the pathogenesis of sepsis could represent a new avenue to improve its diagnosis. The aims of this study are 1) to quantify immunological dysfunction in surgical patients with presence/absence of sepsis using a droplet digital PCR (ddPCR) transcriptomic analysis. 2) To evaluate this approach for improving identification of sepsis in these patients.

Methods

Expression of the neutrophil protease gene (LCN2/NGAL) and also of a gene involved in the immunological synapse (HLADRA) were quantified in blood from 101 surgical patients with sepsis, 53 uninfected surgical patients and 16 blood donors by using ddPCR. Areas under receiver operating characteristic curves (AUROC) and multivariate regression analysis were employed to test the ability of the LCN2/HLADRA ratio to identify sepsis, in comparison with procalcitonin. Results

Sepsis induced over-expression of a neutrophil protease gene (LCN2) and depressed expression of an immunological synapse gene (HLADRA). LCN2/HLA-DRA outperformed procalcitonin in differentiating between patients with sepsis and surgical controls in the AUROC analysis (Fig. 29): 0.90 [0.85 - 0.96], PCT: 0.80 [0.73 - 0.88] (AUROC, [Cl95\%]), and also in the multivariate analysis: LCN2/HLA-DRA: 8.57 [2.25 - 32.62]; PCT: 4.20 [1.15 - 15.43] (OR [Cl95\%]) (Table 9). Gene expression levels of HLA-DRA were an independent marker of hospital mortality.

Conclusions

Quantifying the transcriptomic ratio LCN2/HLA-DRA by ddPCR is a promising approach to improve sepsis diagnosis in surgical patients.

\section{Acknowledgements}

We thank the "Instituto de Salud Carlos III" and "Consejeria de Sanidad de Castilla y Leon" for their financial support, grant numbers [EMER 07/050], [PI13/02110]. [PI15/01451], [PI16/01156]. 


\section{References}

1. Bermejo-Martin, J. F. et al. Defining immunological dysfunction in sepsis: A requisite tool for precision medicine. J. Infect. 72, 525-536 (2016).

2. Singer, M. et al. The Third International Consensus Definitions for Sepsis and Septic Shock (Sepsis-3). JAMA 315, 801-810 (2016).

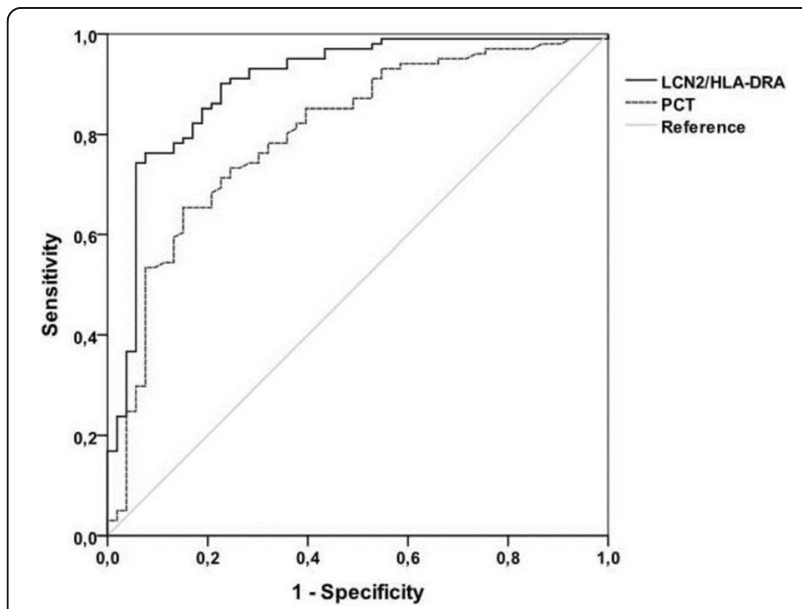

Fig. 29 (abstract P42). AUROC to differentiate sepsis patients from surgical controls. Comparison between LCN2/HLA-DRA and PCT AUROCs to differentiate sepsis

Table 9 (abstract P42). Diagnostic accuracy of the LCN2/HLA-DRA for diagnosis of sepsis

\begin{tabular}{|c|c|c|c|c|c|c|c|c|c|c|c|}
\hline \multirow[b]{3}{*}{$\begin{array}{l}\text { LCN2/ } \\
\text { HLA- } \\
\text { DRA }\end{array}$} & \multicolumn{7}{|c|}{ AUROC } & \multicolumn{4}{|c|}{$\begin{array}{l}\text { Multivariate analysis for } \\
O O P\end{array}$} \\
\hline & \multirow{2}{*}{$\frac{\text { Area }}{0.89}$} & \multicolumn{2}{|c|}{ [C195\%] } & \multirow{2}{*}{$\begin{array}{l}p \\
<0.001\end{array}$} & \multirow{2}{*}{$\frac{O O P}{0.1991}$} & \multirow{2}{*}{$\frac{\mathrm{Se}}{0.837}$} & \multirow{2}{*}{$\frac{S p}{0.811}$} & \multirow{2}{*}{$\begin{array}{l}\text { OR } \\
8.9\end{array}$} & \multicolumn{2}{|c|}{ C195\% } & \multirow{2}{*}{$\begin{array}{l}p \\
0.002\end{array}$} \\
\hline & & 0.83 & 0.95 & & & & & & 2.2 & 36.9 & \\
\hline PCT & 0.80 & 0.73 & 0.88 & $<0.001$ & 1.11 & 0.733 & 0.755 & 6.7 & 1.7 & 26.5 & 0.007 \\
\hline
\end{tabular}

Left: Areas under receiving operating curves (AUROC) for differential diagnosis between sepsis patients and surgical controls. OOP: optimal operating point; Se: sensitivity; Sp: specificity. Right: Multivariate analysis for diagnosing the presence of sepsis. Adjusting variables were [SOFA score], [age], [urgent surgery], [chronic renal failure], [Cancer], [Diabetes Mellitus], [Cardiovascular Disease]

\section{P43}

Prognostic value of basophils in septic shock patients

Marta Martín-Fernández', Raquel Almansa', Eduardo Tamayo²,

David Andaluz-Ojeda ${ }^{3}$, Jesús Blanco ${ }^{4}$, Arturo Muriel ${ }^{4}$,

Ruben Herran-Monge ${ }^{4}$, María Heredia-Rodríguez², Esther Gómez-Sánchez², Jesús F Bermejo-Martín

${ }^{1}$ Group for Biomedical Research in Sepsis (Bio·Sepsis), Hospital Clínico Universitario de Valladolid/IECSCYL, Valladolid, Spain; ${ }^{2}$ Anesthesiology and Reanimation Service, Hospital Clínico Universitario de Valladolid, Valladolid, Spain; ${ }^{3}$ Intensive Care Medicine Service, Hospital Clínico Universitario de Valladolid, Valladolid, Spain; ${ }^{4}$ ntensive Care Medicine Service, Hospital Universitario Río Hortega, Valladolid, Spain

Correspondence: Marta Martín-Fernández (martamf6@hotmail.com) Intensive Care Medicine Experimental 2017, 5(Suppl 1):P43

\section{Background}

Septic shock is defined as a subset of sepsis in which underlying circulatory, cellular, and metabolic abnormalities are associated with a greater risk of mortality than sepsis alone [1]. Septic syndrome represents the major cause of death among critically ill patients worldwide, constituting an important public health problem [2]. In this sense, leukocytes and its subpopulations are crucial components of the innate immune response and the first line of defense against infection [3]. The role of the different leukocyte subsets in the prognosis of severe infections has been studied in different works but these studies have somehow forgotten the basophil. The aim of this study was to evaluate the ability of basophil count in blood to predict mortality in septic shock.

Methods

We performed a two-stage multicenter, retrospective study, aimed at evaluating the potential role of leukocyte subpopulations in patients with septic shock and no immunosuppression. The Derivation Cohort accounted with 238 patients and the Validation Cohort with 186 patients. Statistical analyses were performed to evaluate the impact of basophil count on mortality at 28 days. Results

When patients of the Derivation cohort were distributed into groups based on deciles for basophils count at 28-days for mortality prediction, 110 basophils was selected as cut-off. Patients with $>110$ basophils $/ \mathrm{mm} 3$ died earlier (Fig. 30). Multivariate COX regression analysis demonstrated that showing $>110$ basophils was an independent predictor of mortality $(p=0.048$ and $\mathrm{HR}=2.32$ ) (Table 10).

The cut-off identified in the Derivation Cohort was tested in the Validation Cohort. Kaplan-Meier survival analysis (Fig. 30) and COX regression multivariate analysis confirmed the results obtained in the Derivation cohort $(p=0.012$ and $H R=2.288)$ (Table 11).

Conclusions

Our study reveals a previously unnoticed association between basophil counts and mortality in septic shock.

\section{Acknowledgements}

This study was supported by Proyectos de Investigación en Biomedicina, Consejería de Sanidad, JCYL (BOCYL-D-26072010). This work was also supported by Fondo de Investigaciones Sanitarias, Instituto de Salud Carlos III, Spain (grant numbers: [PI13/02110], [PI16/01156]).

\section{References}

1. Singer, M. et al. The Third International Consensus Definitions for Sepsis and Septic Shock (Sepsis-3). JAMA 315, 801-810 (2016).

2. Reinhart, K. et al. Recognizing Sepsis as a Global Health Priority - A WHO Resolution. N. Engl. J. Med. (2017). doi:10.1056/NEJMp1707170

3. Demaret, J. et al. Marked alterations of neutrophil functions during sepsis-induced immunosuppression. J. Leukoc. Biol. 98, 1081-1090 (2015).

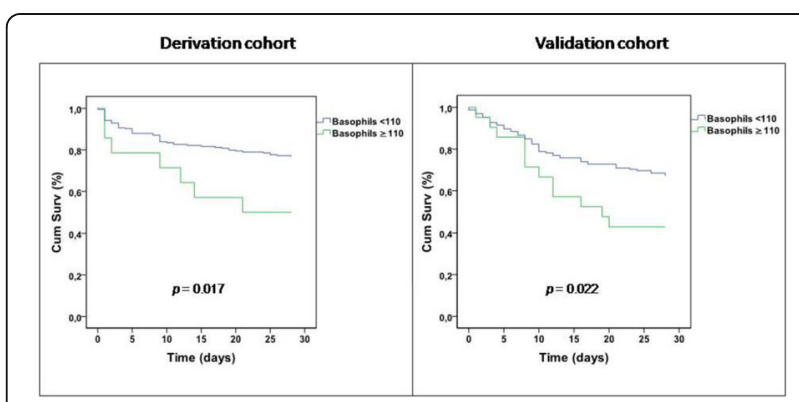

Fig. 30 (abstract P43). Kaplan-Meier survival analysis of 110 basophils cut-off in both Derivation and Validation cohorts

Table 10 (abstract P43). Multivariate COX regression analysis of 110 basophils cut-off in Derivation cohort

\begin{tabular}{lllll}
\hline & HR & $95 \% \mathrm{Cl}$ & & $\mathrm{p}$-Value \\
\hline Age & 1.01 & 0.99 & 1.04 & 0.240 \\
APACHE II & 1.07 & 1.03 & 1.10 & 0.000 \\
COPD & 1.46 & 0.74 & 2.89 & 0.278 \\
Mechanical Ventilation & 2.77 & 1.22 & 6.28 & 0.015 \\
Gram + & 0.55 & 0.27 & 1.15 & 0.111 \\
Fungi & 2.55 & 0.98 & 6.66 & 0.055 \\
Basophils $\left(>110\right.$ cells $\left./ \mathrm{mm}^{3}\right)$ & 2.32 & 1.01 & 5.34 & 0.048 \\
\hline
\end{tabular}


Table 11 (abstract P43). Multivariate COX regression analysis of 110 basophils cut-off in Validation cohort

\begin{tabular}{lllll}
\hline & HR & $95 \% \mathrm{Cl}$ & & p-Value \\
\hline Age & 1.03 & 1.00 & 1.06 & 0.046 \\
APACHE II & 1.05 & 1.00 & 1.09 & 0.043 \\
Diabetes & 0.54 & 0.24 & 1.18 & 0.121 \\
Gram + & 0.56 & 0.32 & 0.99 & 0.045 \\
Basophils $\left(>110\right.$ cells $\left./ \mathrm{mm}^{3}\right)$ & 2.29 & 1.20 & 4.37 & 0.012 \\
\hline
\end{tabular}

\section{P44}

Withdrawn

\section{P45}

Inhibition of the amyloidogenic pathway decreases long-term cognitive damage in rats submitted to sepsis

Cleonice M Michelon, Pricila R M Ávila, Monique Michels, Beatriz Sonai, Henrique Burger, Alexandre Piccinini, Felipe Dal Pizzol

Laboratory of Experimental Pathophysiology, Graduate Program in Health Sciences, University of Southern Santa Catarina-UNESC, Criciúma, SC, Brazil

Correspondence: Cleonice M Michelon (c_michelon@hotmail.com)

Intensive Care Medicine Experimental 2017, 5(Suppl 1):P45

\section{Background}

Sepsis can be defined as the life-threatening organ dysfunction due to a dysregulated host response to infection[1,2]. Acute systemic inflammation can activate the innate immune system, launching a cascade of physiological and behavioral changes ultimately affecting the central nervous system (CNS)[3]. Some of these CNS alterations resemble the pathophysiological mechanisms of neurodegenerative diseases[4,5]. Animals submitted to sepsis induced by cecal ligation and puncture showed increased amyloid beta $(A \beta)$ peptide deposition in brain structures, and this fact is related to cognitive impairment[4]. In this context, the aim of this study was evaluated the effect of inhibition of the amyloidogenic pathway on the deposition of beta-amyloid peptide in brain structures and on the behavior of animals submitted to sepsis.

Methods

Male, Wistar, adult rats were used to perform the experiment. Initially, the activity of secretases in brain structures of septic and non-septic animals was determined. Sepsis was induced using the technique of cecal ligation and perforation (CLP). Next, the animals were divided into three groups: Sham, Sepsis and Sepsis + Inhibitor. The sepsis + inhibitor group received treatment with a beta or gamma secretase, or $A \beta$ inhibitor on the 7th, 8th and 9th day after induction of sepsis. The animals were submitted to behavioral test to evaluate memory retention on the 15th day after induction and later sacrificed to remove structures, prefrontal cortex and hippocampus, for $A \beta$ determination by Western Blot. Data were analyzed using SPSS with $95 \%$ confidence interval.

Results

The activity of beta and gamma secretase were significantly increased in prefrontal cortex and hippocampus 10 and 30 days after sepsis induction, supporting the hypothesis that neuroinflammation promoted by sepsis stimulates Amyloid Precursor Proteins cleavage for amyloidogenic pathway (Fig. 31). Animals treated with all inhibitors when submitted to the inhibitory avoidance test, showed significantly longer latency time than the control group, demonstrating that inhibitors treatments prevents cognitive damage (Fig. 32). In animals treated by betasecretase inhibitor, $A \beta$ peptide was significantly lower in hippocampus in 15 days and in 30 days was observed reduction in prefrontal cortex deposition, when compared to control (Fig. 33), suggesting that the inhibition of enzymes participating in the amyloidogenic pathway can reduce $A \beta$ peptide deposition in brain structures.

Conclusions

We conclude that inhibition of the amyloidogenic pathway decreases $A \beta$ peptide expression in brain structures reducing long-term cognitive damage due to sepsis.

\section{Acknowledgements}

Funding: This work was supported by CAPES, FAPESC, CNPQ and UNESC.

\section{References}

1. Seymour CW, Liu VX, Iwashyna TJ, et al.: Assessment of Clinical Criteria for Sepsis: For the Third International Consensus Definitions for Sepsis and Septic Shock (Sepsis-3). [Internet]. JAMA 2016; 315:762-774

2. van der Poll T, van de Veerdonk FL, Scicluna BP, et al.: The immunopathology of sepsis and potential therapeutic targets [Internet]. Nat Rev Immunol 2017; Available from: http://www.nature.com/doifinder/10.1038/nri.2017.36

3. Fu $H Q$, Yang $T$, Xiao $W$, et al:: Prolonged Neuroinflammation after Lipopolysaccharide Exposure in Aged Rats. PLoS One 2014; 9(8): e106331

4. Schwalm MT, Pasquali M, Miguel SP, et al.: Acute brain inflammation and oxidative damage are related to long-term cognitive deficits and markers of neurodegeneration in sepsis-survivor rats. Mol Neurobiol 2014; 49:380-385

5. Cunningham C, Hennessy E: Co-morbidity and systemic inflammation as drivers of cognitive decline: new experimental models adopting a broader paradigm in dementia research. Res Ther 2011; 7:33

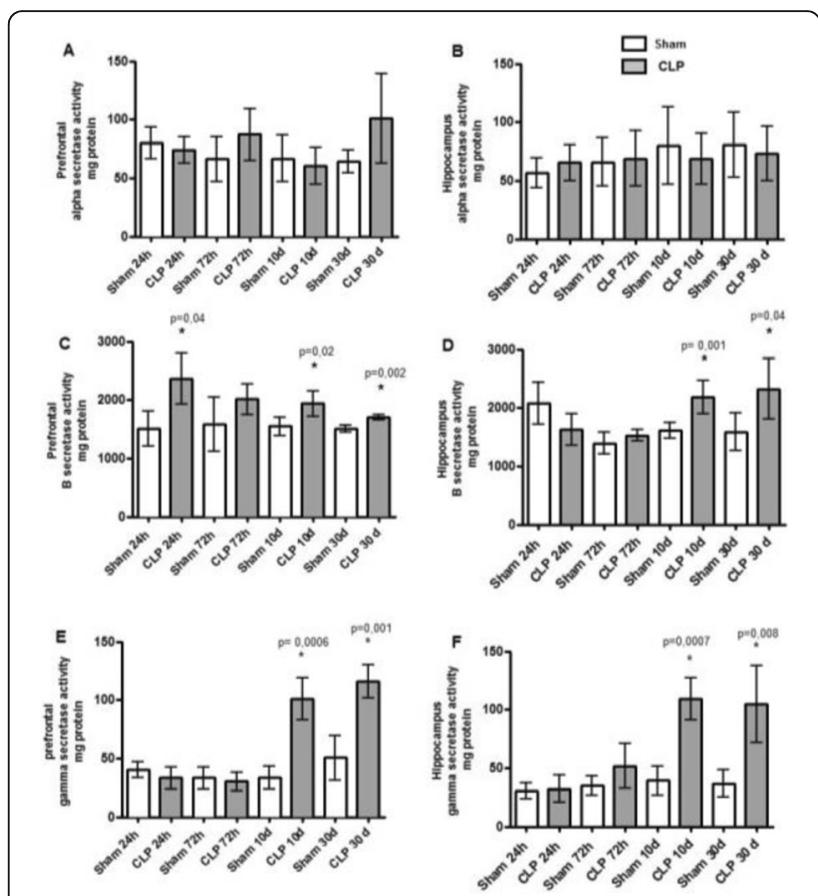

Fig. 31 (abstract P45). Secretases activity determination in prefrontal cortex and hippocampus of rats submitted to sepsis 

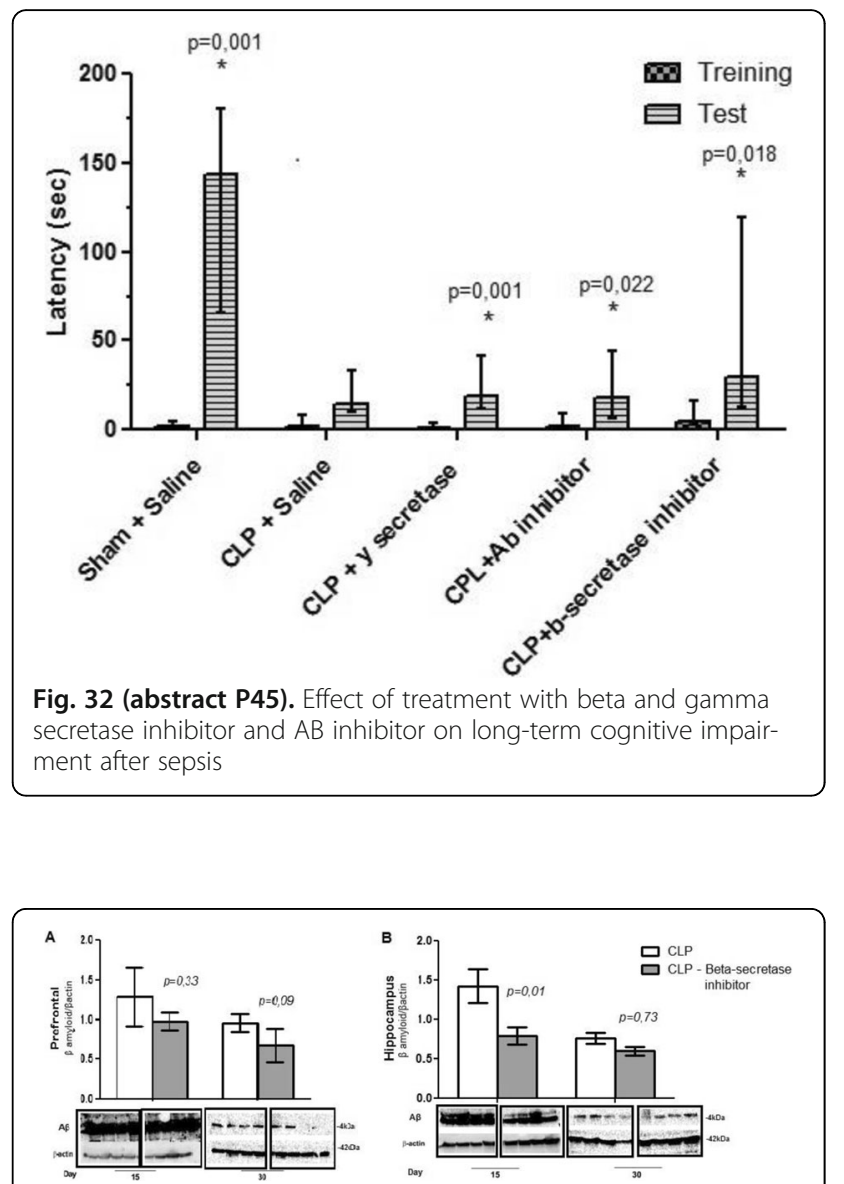

Fig. 33 (abstract P45). A peptide expression in brain structures of septic rats treated and untreated with beta-secretase inhibitor

\section{P46}

Life-space assessment score in elderly patients with sepsis: a single center retrospective cohort study

Ryo Ueno', Atsushi Shiraishi ${ }^{2}$, Ryohei Yamamoto', Seibi Kobara ${ }^{3}$, Yoshiro Hayashi ${ }^{1}$

${ }^{1}$ Department of Intensive Care Medicine, Kameda Medical Center, Kamogawa, Japan; ${ }^{2}$ Emergency and Trauma Center, Kameda Medical Center, Kamogawa, Japan; ${ }^{3}$ Department of Rehabilitation, Kameda Medical Center, Kamogawa, Japan

Correspondence: Ryo Ueno (rueno-tky@umin.ac.jp)

Intensive Care Medicine Experimental 2017, 5(Suppl 1):P46

\section{Background}

Life-Space Assessment Score (LSA) [1] is an established metrics of mobility by asking about movement in areas ranging from one's bedroom $(L S A=0)$ to beyond one's town $(L S A=5)$. Among elderly patients with sepsis, physiological age such as mobility function is often different from their chronological age. We hypothesized that LSA is a risk factor for 28-day mortality in elderly patients with sepsis, independent of their chronological age.

Methods

Design: A single-center retrospective cohort study

Objective: To determine whether baseline restricted life-space is associated with 28-day mortality in the elderly patients with sepsis Setting: Mixed-ICU with closed system, tertiary and teaching hospital in Kamogawa City in Japan, where population aging rate (age $>=$ 65 years old) is higher than $30 \%$ [2].

Population: All elderly (age $>=65$ years old) patients with sepsis admitted to the ICU between September 2014 and January 2017 were included. Sepsis was diagnosed with Sepsis-3 criteria [3].
Primary Outcome: 28-day mortality

Statistical methods: Multivariate logistic regression after adjustment for confounders (age, gender, APACHE2 score, Charlson comorbidity index and do-not-attempt-resuscitation code) was used to evaluate the association between LSA and the 28-day mortality.

Results

Of the 314 participants, 72 (22.9\%) died at 28 days. Among survivors and non-survivors, mean LSA was $2.85(\mathrm{SD}=1.73)$ and $2.25(\mathrm{SD}=$ 1.63) $(p=0.009)$, respectively. The results of multivariate analysis and sensitivity analysis are summarised in Table 12.

Conclusions

Although not statistically significant, low LSA score might be associated with 28-day mortality among elderly patients with sepsis, independent of age. These findings suggest a need for retrospective trial with larger sample size.

\section{References}

1) Baker PS, Bodner EV, Allman RM. Measuring life-space mobility in community-dwelling older adults. J Am Geriatr Soc. 2003 Nov;51(11):1610-4.

2) [https://www.pref.chiba.lg.jp/kenzu/seikatsushuukan/documents/ 21 kamogawashi.pdf] Accessed 29 August 2016.

3) Shankar-Hari M, Phillips GS, Levy ML, et al.; Sepsis Definitions Task Force. Developing a New Definition and Assessing New Clinical Criteria for Septic Shock: For the Third International Consensus Definitions for Sepsis and Septic Shock (Sepsis-3). JAMA. 2016 Feb 23;315(8):775-87.

Table 12 (abstract P46). Multivariable Analyses of Association of LSA at Admission with Mortality

\begin{tabular}{lll}
\hline Analysis & OR per 1 point Increase in LSA (95\% Cl) & P-value \\
\hline Primary Analysis* & $0.97(0.94,1.00)$ & 0.086 \\
Secondary Analysis & & \\
SAPS-II adjusted model & $0.97(0.94,1.00)$ & 0.076 \\
SOFA adjusted model & $0.97(0.94,1.00)$ & 0.098 \\
mixed effect model & $0.86(0.66,1.10)$ & 0.230
\end{tabular}

*Adjusted for age, gender, APACHE 2 score, charlson comorbidity index, DNAR code

\section{P47}

Inter-rater reliability of the SOFA score; a single center retrospective observational study

Ryohei Yamamoto ${ }^{1}$, Natsuki Kondo ${ }^{1}$, Ryo Ueno' ${ }^{1}$ Yasuda Hideto ${ }^{1}$

Atsushi Shiraishi ${ }^{2}$, Yoshiro Hayashi ${ }^{1}$

${ }^{1}$ Department of Intensive Care Medicine, Kameda Medical Center, Kamogawa, Japan; ${ }^{2}$ Emergency and Trauma Center, Kameda Medical

Center, Kamogawa, Japan

Correspondence: Ryohei Yamamoto (ryoheiyamamoto11@gmail.com) Intensive Care Medicine Experimental 2017, 5(Suppl 1):P47

\section{Background}

Since the introduction of sepsis-3[1], SOFA score[2,3] has become increasingly popular in intensive care. However, SOFA score has subjective and complicated components, which may cause inter-rater variability.

\section{Methods}

Objective: To evaluate the inter-rater reliability of SOFA score in ICU patients with sepsis

Design: Single center retrospective observational study

Patients: 491 consecutive patients who were diagnosed with sepsis by the 2001 international diagnostic criteria (sepsis-2) admitted to ICU between April 2013 and March 2016

Setting: A mixed ICU with closed system in Kameda Medical Center (Kamogawa, Japan)

Procedure: Two independent observers, who were senior intensive care trainees (R.Y. and N.K.), assessed SOFA score retrospectively. 
SOFA score was calculated from the worst values in the 24 hourperiod after ICU admission obtained from electronic medical record. Any other detailed rules for scoring SOFA was not made. Primary Outcome: Inter-rater agreement of SOFA score between two evaluators

Statistics: Categorical variables were described as numbers and proportions. Continuous variables were described as mean and standard deviation or median and inter-quartile range. Inter-class correlation coefficients were used to evaluate inter-rater reliability between two assessors for SOFA score.

Results

431 patients were included in the analysis. Patient characteristics were summarized in Table 13. The inter-rater reliability of SOFA score was high (ICC $0.82,95 \% \mathrm{Cl} ; 0.72-0.88)$. The inter-rater reliability of central nervous system component was moderate $(0.49,95 \% \mathrm{Cl} ; 0.34-$ 0.61 ). Detailed results by each component of SOFA score were shown in Table 14.

Conclusions

Inter-rater reliability of SOFA score was high, although that of CNS component was moderate. More rigorous scoring rules for the CNS component of SOFA score may insure further objectivity of SOFA score.

\section{References}

1)Singer

M, Deutschman CS, Seymour CW, et al

JC, Martin GS, Opal SM, Rubenfeld GD, van der Poll T, Vincent JL, Angus DC. The Third International Consensus Definitions for Sepsis and Septic Shock (Sepsis-3). JAMA. 2016 Feb 23;315(8):801-10.

2)Vincent

JL, Moreno R, Takala J, et al; Working Group on Sepsis-Related Problems of the European Society of Intensive Care Medicine. The SOFA (Sepsis-related Organ Failure Assessment) score to describe organ dysfunction/failure. Intensive Care Med. 1996;22(7):707-710. 3)Vincent

$J$, de Mendonça A, Cantraine F, et al; Working Group on "Sepsis-Related Problems" of the European Society of Intensive Care Medicine. Use of the SOFA score to assess the incidence of organ dysfunction/failure in intensive care units: results of a multicenter, prospective study. Crit Care Med. 1998;26(11):1793-1800.

Table 13 (abstract P47). Patients demographics

\begin{tabular}{ll}
\hline $\mathrm{n}=431$ & \\
\hline Age, median [IQR] & $75[65-82]$ \\
Female gender, No (\%) & $150(34.8)$ \\
Medical, No (\%) & $303(70.3)$ \\
APACHE II, median [IQR] & $19[15-26]$ \\
SAPS II, median [IQR] & $49[19-49]$ \\
Base line SOFA, median [IQR] & $0[0-1]$ \\
DNAR order on admission, No (\%) & $94(21.8)$ \\
Charlson index, median [IQR] & $2[1-4]$ \\
Mechanical ventilation, No (\%) & $212(49.2)$ \\
Renal replacement therapy, No (\%) & $115(26.7)$ \\
Noradrenaline use, No (\%) & $264(61.3)$ \\
In-ICU mortality, No (\%) & $55(12.8)$ \\
28-day mortality, No (\%) & $81(18.8)$ \\
In-hospital mortality, No (\%) & $119(27.6)$ \\
\hline
\end{tabular}

Table 14 (abstract P47). See text for description

\begin{tabular}{ll}
\hline SOFA & $\begin{array}{l}\text { ICC* } \\
\text { value }(95 \% \mathrm{Cl})\end{array}$ \\
SOFA components & $0.82(0.72-0.88)$ \\
Respiratory & \\
Coagulation & $0.74(0.66-0.80)$ \\
Liver & $0.90(0.88-0.92)$ \\
Cardiovascular & $0.85(0.74-0.84)$ \\
CNSt & $0.79(0.74-0.84)$ \\
Renal & $0.49(0.34-0.61)$ \\
\hline
\end{tabular}

*ICC: Intra-class correlation coefficients

tCNS: Central nervous system

P48

The ability of Heparin-Binding Protein to identify delayed shock in Emergency Department sepsis patients is impacted by age and source of infection

Brett Schuchardt ${ }^{1,2}$, Muge Capan', Adam Linder ${ }^{3}$, Per Åkesson ${ }^{3}$, Rebecca Kowalski, ${ }^{1,2}$, Kristen Miller ${ }^{4}$, Ryan Arnold ${ }^{1,2}$

${ }^{1}$ Value Institute, Christiana Care Health System, Newark, DE, USA;

${ }^{2}$ Department of Emergency Medicine, Christiana Care Health System,

Newark, DE, USA; ${ }^{3}$ Department of Clinical Sciences, Division of Infection

Medicine, Skåne University Hospital, Lund University, Lund, Sweden;

${ }^{4}$ National Center for Human Factors in Healthcare, MedStar Institute for Innovation, Washington DC, USA

Correspondence: Brett Schuchardt (brett.schuchardt10@gmail.com)

Intensive Care Medicine Experimental 2017, 5(Suppl 1):P48

\section{Background}

Within Emergency Department (ED) sepsis patients, delayed deterioration resulting in shock is associated with an increased risk of death. Heparin-binding protein (HBP), a neutrophil-released serum biomarker, has been shown to be elevated in patients developing delayed shock. The objective of this study was to assess the performance of HBP to predict delayed shock among sepsis sub-populations.

Methods

This was a secondary analysis of a pooled database of patients enrolled into one of two prospective, multi-center observational studies of adult patients from five Swedish and two United States EDs admitted with suspected or confirmed infection. HBP levels were drawn upon ED arrival. Patients were excluded if they were hypotensive [systolic blood pressure $(\mathrm{sBP})<90 \mathrm{mmHg}$ ] upon ED arrival. The primary outcomes were: 1 ) early shock (eShock) $=$ any $\mathrm{sBP}<90$ within 12 hours and 2 ) delayed shock (dShock) = any sBP $<90$ that occurred between 12 and 72 hours after arrival. Controls were defined as patients without shock within 72 hours from arrival. Sepsis subpopulations were defined a priori by age and source of infection. Univariate logistic regression models were utilized to identify independent predictors associated with each outcome.

Results

Over 34 months, 1055 patients were enrolled, with 962 for this analysis after exclusion criteria applied. HBP levels were significantly higher in eShock $(\mathrm{p} 30 \mathrm{ng} / \mathrm{mL})$ was associated with progression to both eShock [OR $=3.67 ; 95 \% \mathrm{Cl}[2.10,6.27], \mathrm{p}<0.0001]$ and dShock $(\mathrm{OR}=2.29 ; 95 \% \mathrm{Cl}[1.33,4.02], \mathrm{p}=0.0032)$. HBP levels in patients without shock increased with age. HBP was elevated across all age groups $<80$ for development of eShock $(<40$ : $p<0.0001 ; 40-59$ : $p=$ 0.007; 60-79: $p<0.0001)$ and dShock $(<40: p=0.025 ; 40-59: p=0.012$; 60-79: $p=0.011)$. In identifying eShock, HBP values were increased across all infection types except abdominal (pneumonia: $p<0.0001$; skin: $p=0.0002$; urinary: $p=0.0007$; other: $p=0.024)$. In identifying dShock, HBP levels were increased for urinary $(p=0.004)$ and abdominal $(p=$ 0.004 ) sources but not pneumonia, skin, and other $(p=N S)$ (Fig. 35). 


\section{Conclusions}

An elevated HBP is associated with a high risk of progression to shock within 72 hours of ED arrival. Differences seen in HBP levels based on age and infection type support the need for further study into the impact of co-morbid and disease specific factors on predictive ability and clinical interpretation of all biomarkers in sepsis.

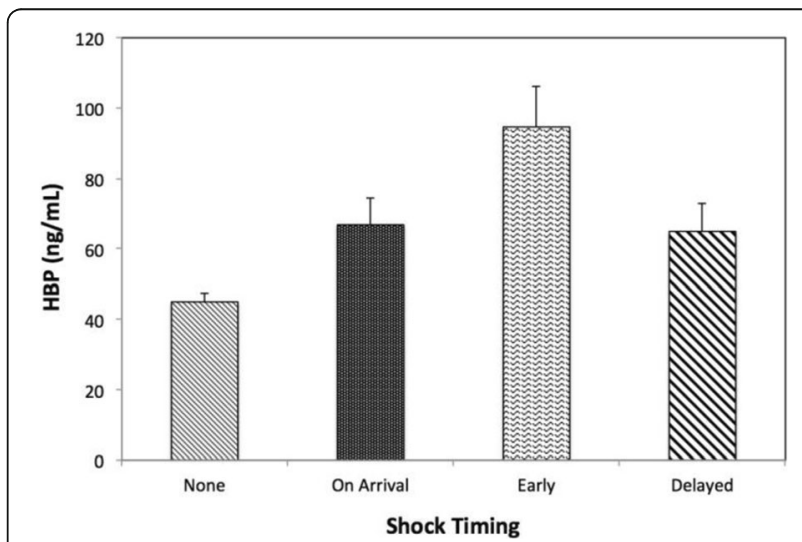

Fig. 34 (abstract P48). See text for description

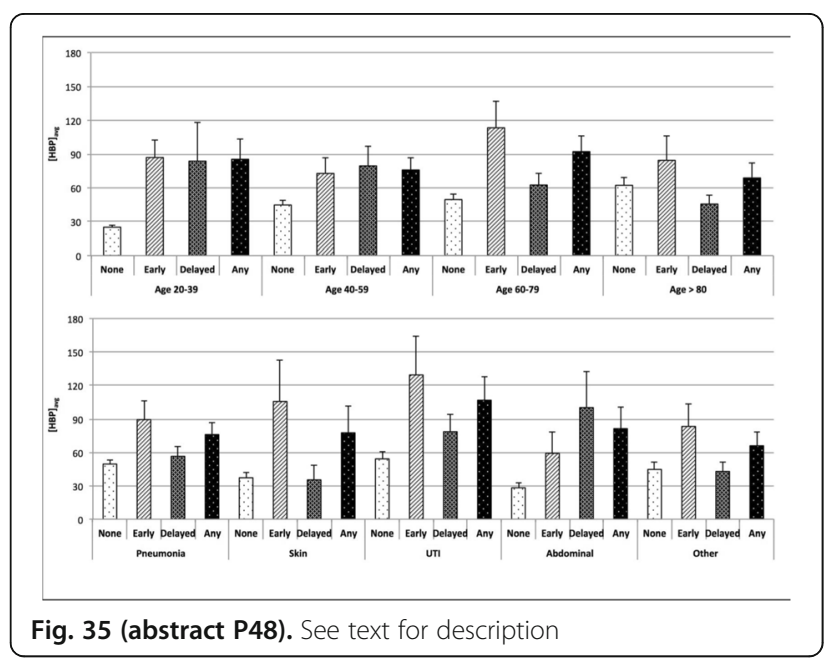

P49

Outcomes associated with the compliance of mandated weight-based fluid resuscitation for septic shock

Rebecca Kowalski', Yash Pandey ${ }^{1}$, Kristen Miller², Pan Wu', Brett Schuchardt', Ryan Arnold

${ }^{1}$ Christiana Care Health System, Value Institute, Newark, DE, USA; ${ }^{2}$ National Center for Human Factors in Healthcare, MedStar Institute for Innovation, Washington DC, USA

Correspondence: Rebecca Kowalski

(rebecca.kowalski@christianacare.org)

Intensive Care Medicine Experimental 2017, 5(Suppl 1):P49

This abstract is not included here as it has already been published [1].

\section{Reference}

[1] Pandey Y, Kowalski R, Miller K, and Arnold R. SAEM 2017 Annual Meeting Abstracts: (180) Outcomes associated with the compliance of mandated weight-based fluid resuscitation for septic shock. Academic Emergency Medicine. (2017) Vol 24 S1
P50

Infections and organ failures: a single center study of prevalence and mortality

Muge Capan', Stephen Hoover', Julie Ivy², Jeanne Huddleston ${ }^{3}$,

Kristen Miller ${ }^{4}$, Ryan Arnold ${ }^{1}$

${ }^{1}$ Christiana Care Health System, Value Institute, Newark, DE, USA;

${ }^{2}$ Edward P. Fitts Department of Industrial and Systems Engineering,

North Carolina State University, Raleigh, NC, USA; ${ }^{3}$ Mayo Clinic Rochester,

Center for Innovation, Rochester, MN, USA; ${ }^{4}$ National Center for Human

Factors in Healthcare, MedStar Institute for Innovation, Washington DC, USA

Muge Caplan (Muge.Capan@ChristianaCare.org)

Intensive Care Medicine Experimental 2017, 5(Suppl 1):P50

\section{Background}

The primary cause of preventable death of hospitalized patients within the sepsis spectrum is the failure to recognize and rescue patients from unexpected acute physiologic deterioration. Organ function in respiratory and cardiovascular systems is measured frequently during patient stay and both are managed aggressively, especially in the infection population. We aim to determine the impact of dysfunction across several additional organ systems on the risk of inpatient mortality.

Methods

Using retrospective EHR data from Christiana Care Health System from July 2013 to December 2015, we investigated the association and co-occurrence between several states of organ dysfunction within a defined infection population. Measures of organ dysfunction included one or more measurements within the cardiovascular, respiratory, renal, hematopoietic, metabolic, nervous, and gastrointestinal systems. Univariate and multivariate logistic regression analyses were conducted to determine the association between inpatient mortality and specific organ dysfunction proximate to time of first antibiotic administration.

Results

An infection and measured organ dysfunction sub-population of 47,480 visits were identified from 210,289 visits. The mortality rate for the infection and organ dysfunction sub-population was $2.96 \%$. In univariate analysis, organ dysfunction within each physiological system was positively associated with inpatient mortality. In multivariate analysis, only indicators for white blood cell count, $\mathrm{FiO} 2: \mathrm{SpO} 2$ ratio, and Glasgow Coma Scale verbal score failed to reach statistical significance $(p>0.05)$ when controlling for all other measures of organ dysfunction as well as age, arrival from nursing home, and administration of antibiotics within 24 hours of arrival. Organ dysfunctions measured by $\mathrm{FiO} 2>21 \%(\mathrm{OR}=4.21)$, lactate $>2 \mathrm{mmol} / \mathrm{L}(\mathrm{OR}=2.37)$, and a relative increase in creatinine of $\geq 50 \%(O R=1.99)$ had the strongest association with mortality.

Conclusions

Organ dysfunction within an infected population is positively associated with inpatient mortality within the sepsis domain. Several measures organ dysfunctions, including relative increase in creatinine and bilirubin $>2 \mathrm{mg} / \mathrm{dL}$, while associated with increased risk of mortality, are not commonly prioritized when evaluating patient severity. Further study is needed to determine if clinician's awareness of risk associated with less appreciated measures will impact patient deterioration.

\section{P51}

So you think you have data - an operational framework for an EHR-based sepsis solution

Muge Capan', Julie Ivy², Jeanne Huddleston ${ }^{3}$, Kristen Miller',

Ryan Arnold'

${ }^{1}$ Christiana Care Health System, Value Institute, Newark, DE, USA:

${ }^{2}$ Edward P. Fitts Department of Industrial and Systems Engineering, North Carolina State University, Raleigh, NC, USA; ${ }^{3}$ Mayo Clinic Rochester, Center for Innovation, Rochester, MN, USA; ${ }^{4}$ National Center for Human Factors in Healthcare, MedStar Institute for Innovation, Washington DC, USA

Correspondence: Muge Caplan (Muge.Capan@ChristianaCare.org) Intensive Care Medicine Experimental 2017, 5(Suppl 1):P51 
Background

While electronic health records (EHRs) inform data-driven clinical decisions involving sepsis recognition and treatment, improper processing of EHR data can threaten any conclusions derived due to compromised data quality. In response to increasing attention on sepsis identification and treatment, we developed an operational framework for addressing challenges in secondary use of EHRs. The objective of this study is to present a systematic approach to assess data quality and address barriers to the operationalization of EHR data in the domain of sepsis.

Methods

Retrospective EHR data from Christiana Care Health System from July 2013 to December 2015 and from the Mayo Clinic from June 2011 to December 2011 was used to develop a framework to identify EHRbased challenges. These EHR-based challenges centered on six data quality attributes: completeness, correctness, concordance, credibility, currency, and clinical relevance.

Results

We developed an operational framework associated with sepsis diagnosis using a dataset with 119,968 and 26,583 unique adult inpatients (210,289 and 33,926 unique visits) from Christiana Care Health System and Mayo Clinic Rochester, respectively. The framework generated recommendations to: (i) define the target clinical environment; (ii) assess six data quality attributes; (iii) design processes to eliminate erroneous documentation; (iv) use appropriate methods to handle missing data; and (v) identify clinically meaningful surrogates. Conclusions

The inherent complexity of diagnosis allows sepsis to uniquely benefit from a comprehensive EHR-based solution. However, false conclusions are unavoidable unless EHR data limitations are addressed. Developing a systematic framework to process EHR data will provide insight into disease progression and enable the operationalization of EHR data in clinical practice.

\section{Acknowledgements}

Authors are presenting on behalf of the S.E.P.S.IS (Sepsis Early Prediction Support Implementation System) Collaborative supported by the National Science Foundation Smart and Connected Health (Award Numbers: 1522072, 1522106, and 1522107).

\section{P52}

Signaling sepsis: understanding clinician mental models for sepsis diagnosis

Kristen Miller ${ }^{1}$, Danielle Mosby ${ }^{1}$, Kendall McKenzie², Russell J Branaghan³ Muge Capan ${ }^{4}$, Brett Schuchardt', Rebecca Kowalski ${ }^{4}$, Joseph Blumenthal', Ella Franklin ${ }^{1}$, Ryan Arnold ${ }^{4}$

${ }^{1}$ National Center for Human Factors in Healthcare, MedStar Institute for Innovation, Washington DC, USA ${ }^{2}$ North Carolina State University, Raleigh NC, USA; ${ }^{3}$ Arizona State University, Mesa, AZ, USA; ${ }^{4}$ Christiana Care Health System, Value Institute, Newark, DE, USA

Correspondence: Kristen Miller (Kristen.E.Miller@medstar.net)

Intensive Care Medicine Experimental 2017, 5(Suppl 1):P52

\section{Background}

In the context of sepsis, challenges to detection and diagnosis are exacerbated by extremely complex cascade of events. Little is known about how evidence-based knowledge is integrated into clinician mental models that often underlie human decision-making. Fuzzylogic cognitive mapping has potential to measure and assess perceptions of sepsis diagnosis both individually and collectively. Taking a human systems engineering approach, the objective of this research is to present differences in mental models among clinicians' diagnosis of sepsis.

Methods

This qualitative study used purposive sampling, a semistructured interview process for data collection, and fuzzy-logic cognitive mapping techniques for analysis. Semi-quantitative models were developed to evaluate the differences between clinician type (e.g. physician, nurse), practice setting (e.g. emergency department, intensive care), and sepsis expertise. Results defined important components in the sepsis diagnosis process, the relationships between these components, and the degree of influence that one component can have on another, defined using qualitative weightings.

Results

Qualitative static models were translated into semi-quantitative dynamic models. Analysis of clinician responses indicated that there was wide variability in clinicians' mental models for sepsis diagnosis but revealed similar themes once responses were organized in a structured terminology platform. Findings also indicated that clinicians identified as "experts" exhibited a clear, focused shared mental model.

Conclusions

Mental models influence how clinicians' behave due to their ability to affect perceptions of reality and can be used to connect values and beliefs that drive organization culture to a commitment to clinical care goals. Addressing variability in terminology and preconceived ideas about sepsis diagnosis will lead to important advances for early diagnoses and appropriate management to improve patient care.

\section{Acknowledgements}

This research project was supported by the National Library of Medicine of the National Institutes of Health under grant number (1R01LM012300-01A1 (PI: Miller)) and by the National Science Foundation Smart and Connected Health (Award Numbers: 1522072, 1522106, and 1522107).

\section{P53}

Severity of illness scoring systems for prognosis and risk stratification of sepsis: a systematic review and comparative analysis

Danielle Mosby', Muge Capan², Kristen Miller', Rebecca Kowalski Brett Schuchardt ${ }^{2}$, Paul Kolm², Rachel Kraft ${ }^{2}$, Ryan Arnold ${ }^{2}$

${ }^{1}$ National Center for Human Factors in Healthcare, MedStar Institute for Innovation, Washington DC, USA; ${ }^{2}$ Christiana Care Health System, Value Institute, Newark, DE, USA

Correspondence: Danielle Mosby (danielle.mosby@gmail.com) Intensive Care Medicine Experimental 2017, 5(Suppl 1):P53

\section{Background}

Multiple scoring systems have been derived and validated with the purpose of quantifying illness severity associated with sepsis and the risk assessment of various outcome measures. The objective of the review was to evaluate the performance features of scoring systems for the prognosis and risk stratification of sepsis and sepsis-induced physiological deterioration.

Material and Methods

A systematic review was conducted in the English language from 1980 to 2016. Papers were included if they contained adults patients with infection with explicit/non-explicit diagnosis of sepsis; were inpatient or emergency department setting; and utilized prognostic modeling. A critical appraisal was conducted for each paper to assess risk of bias. The most common a) ICU-related and b) mortality outcome based on total patients were used to perform a meta-analysis of the pooled discriminatory abilities of scoring systems compared by areas under receiver operating characteristic curve (AUC). Weighted, hierarchical linear regression was used to compare AUCs and results were weighted by sample size. Results

A total of 181 papers met inclusion criteria. 127 unique scoring systems were identified, 15 of which appeared in $>5$ published validation papers and were thus described in detail. A total of 35 independent outcomes were identified, including 14 unique mortality outcomes. The most common shared outcomes were a) admission/ transfer to Intensive Care Unit $(24 / 127 ; 19 \%)$ and b) 30-day inhospital mortality $(14 / 127 ; 11 \%)$. The adjusted AUCs for 30-day inhospital mortality ranged from 0.69 (CRB-65) to 0.92 (MEDS). The adjusted AUCs for ICU admission/transfer to ICU ranged from 0.62 (NHAP) to 0.90 (ATS-rev).

Conclusions

There has been extensive development of unique scoring systems for risk prognostication in sepsis, with no previous systematic comparison of their relative performance. Our results revealed large heterogeneity in outcome measures assessed and risk of bias of each scoring system suggesting the need for further research to 
standardize future metrics. While the high variability identified in the outcome parameters has made large-scale data pooling impossible, results from our meta-analysis show large discrepancy between predictive ability of reported scores and identify that the highest performing scores vary depending on target outcome.
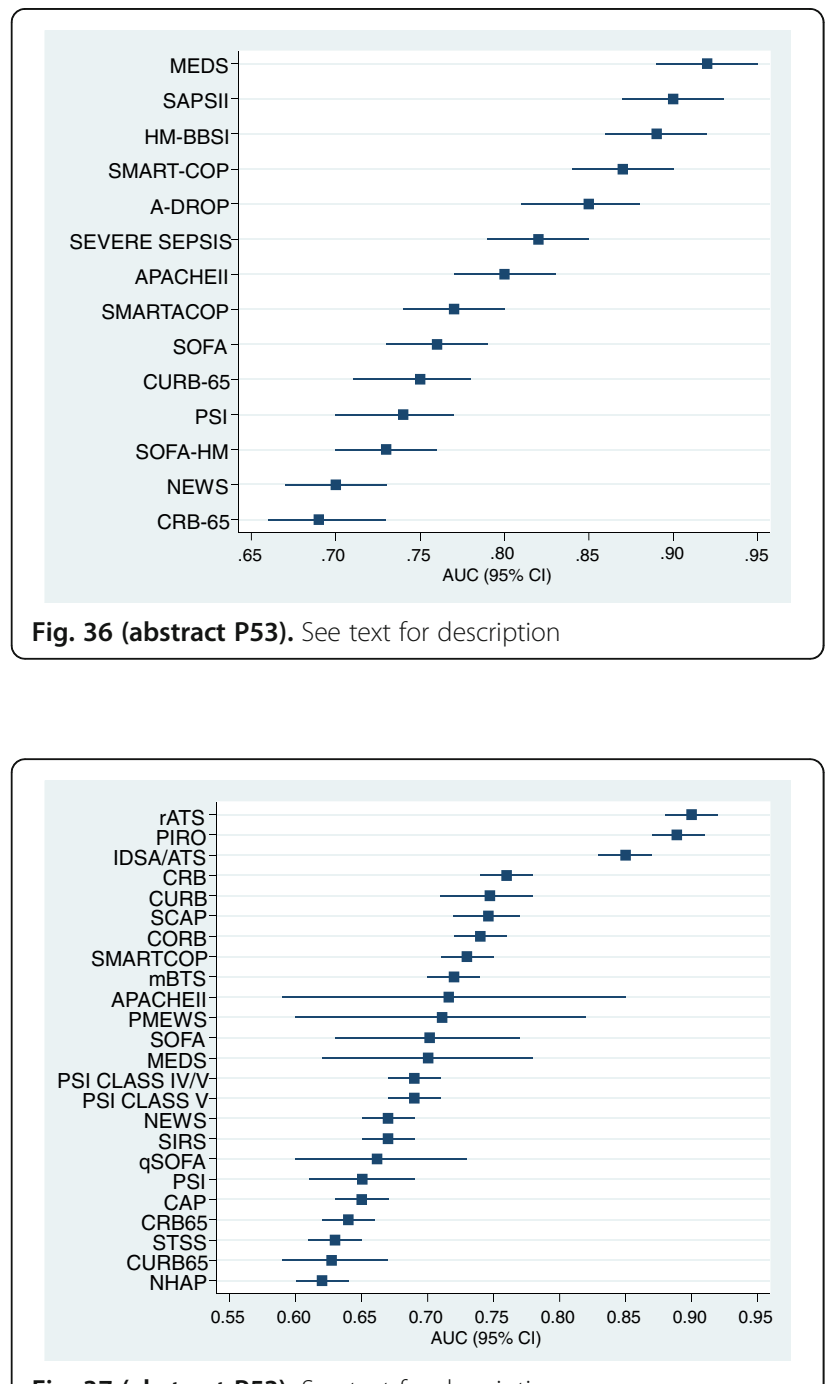

Fig. 37 (abstract P53). See text for description

\section{P54}

Staphylococcus communitary septicaemia in children: study about emergent resistant strains in the paediatric department of a Tunisian university hospital

Houda Ajmi', Khaoula Elfeleh', Sameh Mabrouk', Saida Hassayoun' Minyar Tfifha', Cherifa Chaouech², Noura Zouari', Jalel Chemli',

Noureddine Boujafaar? ${ }^{2}$, Saoussen Abroug ${ }^{1}$

${ }^{1}$ Paediatrics department, Sahloul University Hospital, Sousse, Tunisia;

${ }^{2}$ Bacteriology department, Sahloul University Hospital, Sousse, Tunisia

Correspondence: Houda Ajmi (hd.ajmi@gmail.com)

Intensive Care Medicine Experimental 2017, 5(Suppl 1):P54

Background

Although the commensal colonization of Staphylococcus aureus bacteria showed to be around $30 \%$ of nasal carriage in humans, it is frequently responsible for suppurative communitary infections in infants. Therefore, this bacterium has been associated with recurrent primary skin infections, necrotizing pneumonia and severe osteoarticular infections in young children's. Moreover, because of the worldwide emergence of the community Methicillin-resistant Staphylococcus aureus strain (MRSA), it is expected that incidence of such suppurative pathologies will be subject to a more increase.

Methods

In this retrospective study we examined data collected from 11 years (2006-2017) and involving 30 hospitalized children in the paediatric department of Sahloul university hospital.

Results

Among the 30 patients, there was 16 boys and 14 girls with sex ratio of 1.1. Age ranged between 1 month and 12 years with a mean value of 34 months. We noticed that a congenital heart diseases history was present in two patients while two others suffered from chronic dilated bronchitis. An evident gateway was identified in 25 children: meningitis has been found in 2 cases, acute pulmonary infection in 8 cases, osteomyelitis in 4 cases, facial malignant staphyococi in 2 cases, cutaneous abcess in 2 cases, lymphadenitis in 3 cases, urinary infections in 2 cases, while endocarditis and pericarditis has been detected each in only 1 patient. Staphylococci septicaemia has been identified by blood culture in all our patients' samples. Staphylococus was meticillin-resistant in 4 cases. Under appropriate antibiotherapy, the evoltion was favorable in the majority of cases. However, 4 children have developed a septic shock requiring mechanical ventilation and inotropic placement. In these cases, despite appropriate resisutation care, the outcome was fatal. In 3 children, S. aureus septicemia revealed an underlying pathology: leukemia, diabetes and immunodeficiency in 1 case, respectively.

Conclusions

S.aureus based infections, can be severe and quickly evolve to a life threatening condition, which stresses the importance of an early and adapted management of such infections as the most valuable guarantee of a better patient outcome.

P55

The long pentraxin PTX3 has a non-redundant role in the control of Streptococcus pneumoniae invasive infections

Porte Rémi ${ }^{1}$, Davoudian Sadaf ${ }^{2}$, Asgari Fatemeh ${ }^{2}$, Parente Raffaella ${ }^{2}$, Sironi Marina, ${ }^{2}$ Pasqualini Fabio'2, Barbagallo Maria Luisa², Silva-Gomes Rita ${ }^{2}$ Van der Poll Tom ${ }^{3}$, Garlanda Cecilia², Bottazzi Barbara ${ }^{2}$ and Mantovani Alberto ${ }^{1,2}$

${ }^{1}$ Humanitas University, Rozzano, Milan, Italy; ${ }^{2}$ Humanitas Clinical and Research Hospital, Rozzano, Milan, Italy; ${ }^{3}$ Academic Medical Centre, Center for Experimental and Molecular Medicine and the Division of Infectious Diseases, University of Amsterdam, Amsterdam, Netherlands Correspondence: Porte Rémi (remi.porte@hunimed.eu) Intensive Care Medicine Experimental 2017, 5(Suppl 1):P55

\section{Background}

Pentraxin 3 (PTX3) is a fluid phase pattern recognition molecule, which has served as a paradigm for linking the cellular and humoral arms of innate immunity. PTX3 is an important component of host resistance to pulmonary infections with selected microorganisms, including Aspergillus fumigatus and Pseudomonas aeruginosa. Our aim was to investigate the role of PTX3 in the control of pneumococcal infections caused by Streptococcus pneumoniae, the most common causative bacteria in community-acquired pneumonia and an important cause of mortality world-wide.

Methods

By using a model of invasive pneumococcal infection in mice, we analyzed the local colonization and the bacterial translocation (pneumococcal count in the lung and spleen respectively). PTX3 expression was characterized during the different stages of the infection using different techniques (mRNA analysis, ELISA and immunohistochemistry). Neutrophil recruitment were evaluated by measuring the myeloperoxidase (MPO) levels by ELISA. Binding of PTX3 on S. pneumoniae was realized with a specific assay revealed by Western Blot.

Results

A strong expression of PTX3 is observed at the local site of the infection but also in the systemic compartment following the invasive 
kinetic of the infection, in correlation with an important upregulation of PTX3-inducing cytokines (e.g TNF- $\alpha$ and IL-1 $\beta$ ). With immunohistochemistry analysis we find a specific expression of PTX3 localized at the bacterial site of infection at earlier time points. We also found that human and mouse PTX3 specifically bind on S. pneumoniae, independently of the polysaccharide capsular composition and expression. Comparing the pneumococcal load in PTX3 deficient and competent mice, we observed a higher sensitivity of $P t \times 3^{-/-}$animals during the invasive phase of the infection. Interestingly, the intranasal administration of recombinant PTX3 to Ptx $3^{-/-}$restored the sensitivity to the pneumococcal infection at the level of PTX3 competent mice. We also showed that an exogenous local instillation of PTX3 during the ongoing infection is able to reduce the pulmonary pneumococcal load. Our first results finally indicate that S. pneumoniae opsonized with PTX3 prior the infection are controlled more efficiently in the lung (compared to unopsonized bacteria) but only after the neutrophils recruitment in the respiratory tract.

Conclusions

Our results suggest a non-redundant role of PTX3 in the control of $S$. pneumoniae infections. Given that neutrophils are important for the control of pulmonary pneumococcal infection we are currently characterizing the effect of PTX3 on opsonophagocytosis of S. pneumoniae. This will allow to characterize a possible mechanism involved in PTX3 control of pneumococcal pneumonia.

\section{P56}

Coding and long non-coding RNA networks of specific peripheral blood mononuclear cell subsets in sepsis

Brendon P. Scicluna ${ }^{1,2}$, Arie J. Hoogendijk ${ }^{1}$, Hina Naz-Khan ${ }^{1,2}$,

Lonneke A. van Vught ${ }^{1}$, Maryse A. Wiewel', Janneke Horn ${ }^{3}$,

Marcus J. Schultz ${ }^{3}$, Tom van der Poll ${ }^{1,4}$.

${ }^{1}$ Center for Experimental Molecular Medicine, Academic Medical Center, University of Amsterdam, Amsterdam, the Netherlands; ${ }^{2}$ Department of Clinical Epidemiology, Biostatistics and Bioinformatics, Academic Medical Center, Amsterdam, the Netherlands; ${ }^{3}$ Department of Intensive Care Medicine, Academic Medical Center, University of Amsterdam, Amsterdam, The Netherlands; ${ }^{4}$ Division of Infectious Diseases, Academic Medical Center, University of Amsterdam, Amsterdam, The Netherlands Correspondence: Brendon P. Scicluna (b.scicluna@amc.uva.nl) Intensive Care Medicine Experimental 2017, 5(Suppl 1):P56

\section{Background}

Sepsis is a multifaceted syndrome caused by an unbalanced host response to infection. Peripheral blood mononuclear cells (PBMCs) are crucial effectors of innate and adaptive immune responses, which are functionally impaired and/or hyperactive in sepsis. A comprehensive evaluation of the genomic responses that ensue in specific mononuclear cell subsets of sepsis patients has not been performed. Objective

(1) To identify protein coding and long non-coding RNA (IncRNA) molecules in monocytes, $T$ cells and $B$ cells that are significantly altered due to sepsis; (2) to classify IncRNAs that potentially regulate cell-type specific functions of protein coding transcriptional networks in sepsis. Methods

The study comprised six sepsis patients diagnosed with community acquired pneumonia (CAP) and four healthy subjects. Fluorescence activated cell sorting was used to isolate $\mathrm{CD} 14^{+}$monocytes, $\mathrm{CD} 4^{+} \mathrm{T}$ cells, $C D 8^{+} T$ cells and $C D 19^{+} B$ cells. Total RNA of each cell type was analyzed by RNA-sequencing using Illumina HiSeq 2500. Raw sequences were aligned against the Genome Reference Consortium human genome build 38. DESeq2 was used to compare CAP patients and healthy subjects. Enrichment (Fisher test) of canonical signaling pathways was performed by Ingenuity Pathway Analysis.

Results

Principal component (PC) analysis of protein coding sequence counts in monocytes, $\mathrm{CD}^{+}, \mathrm{CD}^{+} \mathrm{T}$ cells and $\mathrm{B}$ cells revealed clear cellspecific clusters with explainable variance in $\mathrm{PC} 1$ and $\mathrm{PC} 2$ equating to $28 \%$ and $15 \%$, respectively. PC analysis of IncRNA sequences also resulted in clear clusters per cell-type with explainable variance in PC1 and PC2 equating to $19 \%$ and $12 \%$, respectively. Comparing CAP patients to healthy subjects revealed differences that were predominantly unique $(84 \%)$ for monocytes, $\mathrm{CD}^{+}, \mathrm{CD} 8^{+} \mathrm{T}$ cells and $B$ cells. Pathway analysis of different protein coding RNAs uncovered significant enrichment (adjusted $p<0.05$ ) for various cytokine, metabolic, cell growth and death canonical signaling pathways. Notably, a variety of metabolic pathways, for example GDP-glucose biosynthesis, were unique to monocytes; cell death signaling pathways (eg. apoptosis signaling) were unique to $\mathrm{CD}^{+} \mathrm{T}$ cells; antigen presentation and innate-to-adaptive immunity crosstalk pathways were unique to $\mathrm{CD}^{+} \mathrm{T}$ cells. No significant enrichment was detected for unique genes in B cells. Classification of significantly altered IncRNA per cell-type as biotypes revealed enrichment of antisense $(55 \%$, adjusted $p<0.05)$ and long intervening non-coding RNA (lincRNA)(21\%, adjusted $\mathrm{p}<0.05$ ).

Conclusion

Our findings suggest monocytes, $\mathrm{CD} 4^{+}, \mathrm{CD} 8^{+} \mathrm{T}$ cells and $\mathrm{B}$ cells cells engage in predominantly distinct protein coding and IncRNA expression programs in sepsis relative to health. Antisense and long intervening non-coding RNA were predicted as drivers of cell-type specific protein coding gene regulation.

\section{P57}

Identification of blood transcriptional networks dependent on lipopolysaccharide dose in human endotoxemia Hina Naz-Khan ${ }^{1,2}$, Desiree Perlee ${ }^{1}$, Lieke Schoenmaker ${ }^{1}$ Anne-Jan van der Meer ${ }^{1}$, Marek Franitza ${ }^{3}$, Mohammad Toliat ${ }^{3}$, Peter Neurenberg ${ }^{3,4,5}$, Tom van der Poll ${ }^{1,6}$, Brendon P. Scicluna ${ }^{1,2}$

${ }^{1}$ Center for Experimental Molecular Medicine, Academic Medical Center, University of Amsterdam, Amsterdam, the Netherlands; ${ }^{2}$ Department of Clinical Epidemiology, Biostatistics and Bioinformatics, Academic Medical Center, Amsterdam, the Netherlands; ${ }^{3}$ Cologne Center for Genomics (CCG), University of Cologne, Cologne, Germany; ${ }^{4}$ Cologne Excellence Cluster on Cellular Stress Responses in Aging-Associated Diseases (CECAD), University of Cologne, Cologne, Germany; ${ }^{5}$ Center for

Molecular Medicine Cologne (CMMC), University of Cologne, Cologne, Germany; ${ }^{6}$ Division of Infectious Diseases, Academic Medical Center, University of Amsterdam, Amsterdam, The Netherlands

Correspondence: Hina Naz-Khan (h.n.khan@amc.uva.nl)

Intensive Care Medicine Experimental 2017, 5(Suppl 1):P57

Background

The host response to infection is characterized by a complex interplay of cellular transcriptional responses. Both hyper-activation as well as severely depressed host response mechanisms are detrimental to cells and tissue systems. This argues for a better understanding of the quantitative nature of the host response.

Objective:

To identify host innate immune mechanisms that underlie the quantitative genomic response to lipopolysaccharide (LPS) in humans.

Methods

The study included whole blood gene expression microarray data from the human endotoxemia model ( $n=42 ; 4$ hours post-LPS), Publicly available data of Illumina microarrays were retrieved for $1 \mathrm{ng} / \mathrm{kg}$ and $4 \mathrm{ng} / \mathrm{kg}$ LPS doses. We recruited an additional 8 healthy volunteers who received $2 \mathrm{ng} / \mathrm{kg}$ LPS intravenously with blood for microarrays (Affymetrix human genome U219 chips) collected at 4 hours post-LPS. After background correction, the different microarray studies were combined for meta-analysis by firstly re-mapping probes to the current Genome Reference Consortium Human genome build 38 (GRCh38) available via GenCode. Gene expression data were subsequently normalized and adjusted for non-experimental chip effects. Data were analyzed by moderated t statistics and enrichment of canonical signaling pathways performed by Ingenuity Pathway Analysis.

Results

Differential gene expression analysis of $1 \mathrm{ng} / \mathrm{kg}, 2 \mathrm{ng} / \mathrm{kg}$ and $4 \mathrm{ng} / \mathrm{kg}$ LPS doses relative to pre-LPS (baseline) uncovered 6044, 4796 and 5893 significantly altered genes, respectively. Both unique and common transcriptional signatures were identified, with 638 commonly altered and 2267 uniquely altered genes dependent on LPS dose. Pathway analysis of elevated genes as a function of LPS dose revealed 27 canonical signaling pathways 
that included death receptor signaling, protein ubiquitination, $\mathrm{PI} 3 \mathrm{~K} / \mathrm{AKT}$ and IL-6 signaling. Genes with decreased expression as a function of LPS dose significantly over-represented 12 pathways including EIF2 signaling, oxidative phosphorylation and mitochondrial dysfunction.

Conclusion

Shared and distinct cellular biological pathways were identified that responded quantitatively to different LPS doses. Genes involved in translation (EIF2 signaling), metabolism (oxidative phosphorylation, mitochondrial dysfunction) and cell death (death receptor signaling) represented major cellular biological pathways influenced by LPS dose.

\section{P58}

The circulatory small non-coding RNA landscape in health and community-acquired pneumonia

Hina Naz-Khan ${ }^{1,2}$, Lonneke A. van Vught ${ }^{1}$, Aldo Jongejan ${ }^{2}$, Janneke Horn $^{3}$, Olaf L. Cremer ${ }^{4}$, Aeilko H. Zwinderman², Marcus J. Schultz ${ }^{3}$, Marc J. Bonten ${ }^{5,6}$, Tom van der Poll ${ }^{1,7}$, Brendon P. Scicluna ${ }^{1,2}$

${ }^{1}$ Center for Experimental Molecular Medicine, Academic Medical Center, University of Amsterdam, Amsterdam, the Netherlands; ${ }^{2}$ Department of Clinical Epidemiology, Biostatistics and Bioinformatics, Academic Medical Center, Amsterdam, the Netherlands; ${ }^{3}$ Department of Intensive Care Medicine, Academic Medical Center, University of Amsterdam,

Amsterdam, The Netherlands; ${ }^{4}$ Department of Intensive Care Medicine, University Medical Center Utrecht, Utrecht, The Netherlands:

${ }^{5}$ Department of Medical Microbiology, University Medical Center Utrecht, Utrecht, the Netherlands; ${ }^{6}$ Julius Center for Health Sciences and Primary Care, University Medical Center Utrecht, Utrecht, the Netherlands; ${ }^{7}$ Division of Infectious Diseases, Academic Medical Center, University of Amsterdam, Amsterdam, The Netherlands

Correspondence: Hina Naz-Khan (h.n.khan@amc.uva.nl)

Intensive Care Medicine Experimental 2017, 5(Suppl 1):P58

\section{Background}

Community-acquired pneumonia (CAP) is a major determinant of sepsis. Exaggerated systemic cytokine release and inhibitory mechanisms in leukocytes that can lead to immunoparalysis are pathognomonic features of sepsis. Small non-coding RNA, particularly microRNA, are potent modifiers of gene expression that include innate immune signaling genes. Notably, microRNA are not exclusive to the intracellular milieu, but have also been detected in the circulation (cell-free) with functional consequences. A comprehensive evaluation of circulatory small non-coding RNA in CAP has not been performed.

Objective

We here sought to firstly identify differentially abundant circulatory small non-coding RNA in CAP patients as compared to healthy subjects, and secondly delineate those putatively targeted cellular pathways.

Methods

The study comprised 10 intensive care CAP patients with culture proven infection and 5 healthy subjects. Blood plasma (EDTA) was collected for small RNA isolation and processed for small RNA next generation sequencing (Solexa). Sequence alignments were done against the current Genome Reference Consortium Human genome build 38 (GRCh38) available via GenCode.

Results

In health we found 127170 small non-coding RNA sequences, with a preponderance of microRNA (93.5\% of total). Other small RNA species were also detected that included small nucleolar (sno)RNA and small cajal-body (sca)RNA, accounting for $6.5 \%$ of circulatory small RNA species. In CAP patients we found 120943 sequences, with microRNA as the most abundant species (85.2\%). We uncovered 24 differentially abundant circulatory microRNA (13 high, 11 low; adjusted $p<0.05)$ in CAP relative to health. Pathway analysis of the high abundance microRNA revealed an over-representation for PI3KAKT, mTOR, TNF and AMPK signaling pathways, including hsa-miR100, hsa-miR-483 and hsa-miR-501. Low abundance circulatory microRNA were associated to TGF-beta signaling and cell cycle pathways that included hsa-miR-146b, hsa-miR-744 and hsa-miR-191.
Conclusion

In both health and CAP small RNA molecules were detected in the circulation with microRNA the dominant species. Pathway analysis of the differentially abundant microRNA in CAP relative to health suggests metabolic, cytokine and cell cycle pathways may be in the cross-hairs of those circulatory microRNA.

\section{P59}

Justification of the effectiveness of Decasan antiseptic medication in patients with diabetic foot syndrome

Julia Fuss ${ }^{1}$, Anna Voloboyeva ${ }^{2}$

'Department of physical rehabilitation, D.Halycky Lviv National Medical University, Lviv, Ukraine; ${ }^{2}$ Department of intensive care, Central Region Hospital in Pustomyty, Pustomyty, Ukraine

Correspondence: Anna Voloboyeva (voloboyeva@gmail.com)

Intensive Care Medicine Experimental 2017, 5(Suppl 1):P59

\section{Background}

The purulonecrotic lesions of lower limbs is one of the most frequent surgical implications of diabetes mellitus (DM), the results of the treatment of which, unfortunately, have not been satisfactory[1,2]. The diabetes mellitus prevalence in different countries is from 1 to $6 \%[3]$. Methods

The serial industrial samples of Decasan medicinal products of YURiA FARM production and Octinesept Schulke production were used in the researches. The conclusion of antisepsis efficiency was drawn based on whether there was microbial growth in medium after 48 hours of incubation at the temperature of $37^{\circ} \mathrm{C}$. Each indicator was identified according to the results of the test conducted three times. The clinical effectiveness of Decasan was studied in the course of the treatment of 275 inpatients with purulonecrotic lesions.

Results

The index of the minimal bactericidal concentrations fully reflects the sensitivity of a certain strain of microorganisms to the main active ingredient of the antimicrobial remedy when growing in artificial conditions and is taken into account when selecting concentration of antimicrobial compound being part of finished dosage form. The results of the study of sensitivity of clinical strains of microorganisms to Decasan and Octenisept are given in the Table 15.

Analyzing the obtained results, high sensitivity of a wide range of opportunistic microorganisms to the antiseptic medications under study should be pointed out. The sensitivity of individual types to each of the medications, however, significantly differed. Decasan has found wide application in the practice of purulent surgery, where it is used for local treatment of patients with purulent lesions of soft tissues of different localization. Decasan was heated before use to $37-38{ }^{\circ} \mathrm{C}$ and was used to rinse wounds after opening of abscess, phlegmon, carbuncle, boil, whitlow and paraproctitis. The period of complete wound healing was reduced to 2.5 days. The duration of inpatient stay of patients with phlegmons and abscesses reduced from 14.97 and 15.18 days to 12.33 and 10.8 days respectively.

Conclusions

Decasan antiseptic medication has high antimicrobial activity with respect to a wide range of pathogenic and opportunistic microorganisms and potent antiseptic properties, and, therefore, should be used for prevention and treatment of microbial diseases. The experience of the clinical use of Decasan shows its high medical effectiveness in purulonecrotic lesions of different localization, lack of undesired side effects and economic efficiency of a wide medical use.

\section{References}

1. Pedachenko, E. G. Use of a new antimicrobial drug de cametoxin in neurosurgery [Text]/E. G. Pedachenko, V. V. Malovik, G. A. Pedachenko [et al.]//9th Europe an congress of neurosurgery. Book of abstracts. Moscow, 1991. - MKT 19.

2. Fedchuk A.S., Zaritsky V.P., Gridina T.L. Anti influen za and antigerpetic activity of decametoxin//Antiviral Research. - 2003. - V. 57. - P. 137

3. Sepsis and multiple organ disfunction/E.A. Deitch, J.L. Vinsent, W.B. Sounders//London, 2002. - $370 p$ 
Table 15 (abstract P59). Characteristic of antimicrobial activity of serial samples of Decasan and Octenisept

\begin{tabular}{lllll}
\hline Microorganism & Number of strains & \multicolumn{2}{l}{ Antiseptic medication } & \multirow{2}{l}{$\begin{array}{l}\text { Multiplicity of } \\
\text { difference }\end{array}$} \\
\cline { 3 - 4 } & & Decasan & Octenisept & \\
\hline S.aureus & 3 & $4.68 \pm 1.49$ & $16.54 \pm 4.27$ & 3.5 \\
S.epidermidis & 13 & $1.68 \pm 0.60$ & $19.25 \pm 7.09$ & 11.2 \\
E.coli & 5 & $3.68 \pm 0.87$ & $12.69 \pm 3.17$ & 3.5 \\
P.vulgaris & 3 & $12.78 \pm 2.92$ & $26.42 \pm 5.08$ & 2.1 \\
P.aeruginosa & 3 & $91.87 \pm 32.5$ & $78.21 \pm 39.23$ & 1.1 \\
A.fecalis & 3 & $42.62 \pm 8.45$ & $83.45 \pm 16.78$ & 2.0 \\
\hline
\end{tabular}

\section{P60}

Relationship between purulonecrotic diseases in patients with diabetes mellitus and blood sugar level

Julia Fuss', Anna Voloboyeva ${ }^{2}$, Roksolana Yaremkevich ${ }^{3}$

Dmytro Romanchak ${ }^{3}$

'Department of physical rehabilitatioa, D.Halycky Lviv National Medical

University, Lviv, Ukraine; ${ }^{2}$ Department of intensive care Central Region

Hospital in Pustomyty, Pustomyty, Ukraine; ${ }^{3}$ Department of surgery,

Emergensy hospital, Lviv, Ukraine

Correspondence: Julia Fuss (juliafuss78@gmail.com)

Intensive Care Medicine Experimental 2017, 5(Suppl 1):P60

\section{Background}

The problem of detecting purulonecrotic lesions of foot associated with diabetes mellitus remains relevant[1]. It is conditioned by constant increase in the number of patients with this pathology. From the surgical point of view, the significance of this problem is caused by the fact that purulonecrotic processes on foot develop in more than $35-70 \%$ of diabetic patients[2,3]. The diagnostics of purulonecrotic processes in diabetic foot syndrome has recently undergone significant changes[4]. All this points to the relevance of the problem, especially given that early detection of purulonecrotic complications allows, as numerous researches prove, preventing loss of functional capability of lower limbs in $50 \%$ of cases, and thus, lowering economic and social losses of population and country[5, 6].

Methods

The clinical material consisted of 175 patients divided into two groups. The first group included 87 patients with controlled blood sugar level and the second group comprised 88 patients with uncontrolled blood sugar level. The diabetes mellitus type 2 was detected in $86.7 \%$ of patients from the second group and $88.9 \%$ of patients from the first group. The newly diagnosed diabetes mellitus was detected in $7.8 \%$ of patients in the comparison group and $8.1 \%$ of patients in the treatment group. All patients underwent the following tests: complete blood count (WBC, neutrophil (\%), lymphocyte (\%), NLR; LII) and determination of procalcitonin level.

Results

White blood cell counts $(P=0.022)$, neutrophil \% $(P=0.045)$ and the neutrophil lymphocyte ratio (NLR) $(P=0.034)$ were significantly higher in the unregulated DM group compared to that in the regulated DM group (Table 16). There were no differences in lymphocyte $\%$, and LII. NLR were higher in unregulated DM patients with complications than in the regulated DM group. The researchers conducted by us showed that the NLR and LII values in the second group were higher than those in the first group.

Conclusions

Thus, due to the observance of clear correlation between the neutrophil count and blood sugar level, NLR can be considered to be a marker of the development of purulonecrotic diseases in patients with diabetes mellitus, especially with uncontrolled blood sugar level.

\section{References}

1. Levin ME. Foot lesions in patients with diabetes mellitus. End Met Clin North Amer 1996; 25:447-462.
2. Mark P, Sloven Ki. Foot problems in diabetes. Med Clin North Am. 1998; 82 (4):949-71.

3. Laing P. The development and conıplications of diabetic foot ulcers. Am J Surg. 1998 Aug 176 (Suppl 2A): 11-19

4. Levin ME. Foot lesions in patients with diabetes mellitus. End Metab Clin North Am.1996; 5 (2): 448-54

5. Pitsavos C, Tampourlou M, Panagiotakos DB, Skoumas Y, Chrysohoou C Nomikos T, et al: Association Between Low-Grade Systemic Inflammation and Type 2 Diabetes Mellitus Among Men and Women from the ATTICA Study. Rev Diabet Stud 2007, 4:98- 104.

6. Nakanishi N, Sato M, Shirai K, Suzuki K, Tatara K: White blood cell count as a risk factor for hypertension; a study of Japanese male office workers. J Hypertens 2002, 20:851-857.

Table 16 (abstract P60). See text for description

\begin{tabular}{llll}
\hline Characteristic & Group I & Group II & $p$ \\
\hline WBC $\left(\times 10^{9} / \mathrm{L}\right)$ & $7.29 \pm 1.9$ & $8.53 \pm 2.72$ & 0.022 \\
Neutrophile (\%) & $53.27 \pm 8.79$ & $58.58 \pm 9.98$ & 0.045 \\
Lymphocyte (\%) & $34.09 \pm 8.09$ & $32.59 \pm 8.97$ & 0.070 \\
NLR & $1.71 \pm 0.76$ & $2.25 \pm 1.3$ & 0.034 \\
LII & $2.39 \pm 0.2$ & $2.85 \pm 0.29$ & 0.028 \\
PCT & $0.079 \pm 0.08$ & $1.14 \pm 2.65$ & 0.157 \\
\hline
\end{tabular}

P61

Expediency of the different biomarkers use for diagnosis and monitoring of perinatal sepsis treatment of the newborns with surgical pathology

Gennadiy Khanes (androshchuk@hotmail.com)

Neonatal Surgery Department, Ukrainian National Pediatric Hospital

OkhMatDyt, Kyiv, Ukraine

Intensive Care Medicine Experimental 2017, 5(Suppl 1):P61

\section{Background}

The main role in perinatal sepsis appearance is played by intranatal and contamination infections, both bacterial and fungal, and immature immune biologic activity of an organism. The redundant secretion of cytokines (interleukins) happens under the influence of bacterial, viral and fungal toxins, also the humoral and cell immunity is disordered as discharge of bacterial antigens and of complement system and coagulatory hemostasis disorder.

Methods

The experience of treatment of newborns with postoperative and bone and joint sepsis showed that during 3 days of the decease we noted critical values of coagulatory tests such as APTT - more than $50 \mathrm{sec}$, TT - clot absence, PDF - 8.0 and more, Fn $10 \mathrm{ng} / \mathrm{l}$ was observed during intestinal perforation, peritonitis, aspiration pneumonia; it exceeded 2 ng/l during pyelonephritis development against the background of different kidney malformations or tumors. The high level of C-reactive protein (CRP $>24 \mathrm{~mm} / \mathrm{l}$ ) was observed in early stage of the decease in cases of severe sepsis and only in 2 weeks after the beginning of the decease in case of SIRS.

Results

The undertaken monitoring shows that normalization of coagulatory tests is connected with effective surgical treatment, adequate antibacterial therapy, introduction of specific immunoglobulin and to a lesser extent with using protease inhibitors. The main point in diagnostics and treatment of perinatal sepsis is the determination of etiologic factor of bacterial, viral or fungal origin by serologic and PCR methods. The determination was made during the treatment of all patients and allowed to choose the adequate therapy. This investigation showed that bacterial infections caused sepsis in $90 \%$ cases, viral - in $10 \%$. The $30 \%$ of clinical observations showed the joining of fungal infections as well. 
Conclusions

The coagulatory tests, the evaluation of humoral immunity by the presence of specific antibodies and the continuous evaluation of PCT level are the most expedient for early diagnostics of perinatal sepsis in newborns with surgical pathology.

\section{P62}

Extracorporal adsorption of endotoxin for Gram-negative sepsis in oncological patients

Natalia Dmitrievna Ushakova (ndu2000@rambler.ru)

Department of A\&R, Rostov Research Institute of Oncology, Rostov-on-

Don, Russia

Intensive Care Medicine Experimental 2017, 5(Suppl 1):P62

\section{Background}

The aim of the study was to study the clinical efficacy of polymyxin adsorption in gram-negative sepsis in cancer patients.

Methods

The study included 86 patients aged from 47 to 84 , who were operated for oncological diseases of the abdominal cavity, postoperative period was complicated by Gram-negative sepsis (qSOFA $>2, P C T>2 \mathrm{ng} / \mathrm{ml}$, EAA 0.45- 0.9. Microbiological confirmation of sepsis was obtained in $76 \%$ of cases, of which $45 \%$ of the isolated microorganisms were multiresistant. At the time of the diagnosis of sepsis, the severity of the condition by APACHE-II scale was $28.6+3.1$, by SOFA scale: $18.2+4.7$. In 64 of 86 patients $(74.5 \%)$, the development of MNT required organ replacement therapy. All patients received standard sepsis therapy (Surviving Sepsis Campaign: International Guidelines, 2015). Adsorption was performed by immobilized Polymyxin cartridge B "TORAYMYXIN PMX-20R" (Japan). Adsorption was performed with a cartridge with immobilized polymyxin B "TORAYMYXIN PMX-20R" (Japan). The beginning of the procedure is 2-6 hours after the diagnosis of sepsis. Blood flow velocity is $120-150 \mathrm{ml} / \mathrm{min}$, duration is 240 minutes. All patients underwent two sessions of LPS-adsorption at intervals of 24 hours. PCT and EAA were determined before LPS adsorption and 24 hours after each procedure.

Results

After the completion of the selective lipopolysaccharide-adsorption program, optimization of the clinical course of generalized inflammation was observed in $87 \%$ of cases: regression of hyperthermia, a statistically significant decrease in the level of leukocytes and neutrophils in peripheral blood, improvement of biochemical parameters of blood. In $89 \%$ of patients, EAA declined and values were below 0.4. PCT blood levels decreased significantly in $92 \%$ of observations. Against the backdrop of inclusion in the complex intensive treatment of lipopolysaccharide adsorption in $62 \%$ of cases, there was a decrease in the need for vasopressor support in connection with the optimization of the hemodynamic profile. After the final procedure of sorption therapy, 42 of 64 patients had no need for further organ-replacement therapy. LPSadsorption did not cause adverse events. Lethality was $21.9 \%$.

Conclusions

Inclusion of lipopolysaccharide adsorption into intensive therapy of Gram-negative sepsis can effectively stop the manifestation of bacterial endotoxin systemic effects and improve the results of cancer patient hospital treatment period.

\section{P63}

One year follow-up of patients with purulent-necrotic complications of lower limb and sepsis - importance of compliance on postoperative prognosis

Aleksandra V. Shalaeva, Bakhtiyor B. Janabaev, Azam R. Bobabekov,

Nailya A. Dadabaeva, Evgeniya V. Shalaeva

Tashkent Medical Academy, Tashkent, Uzbekistan

Correspondence: Aleksandra V. Shalaeva

(aleksandrashalaeva@gmail.com)

Intensive Care Medicine Experimental 2017, 5(Suppl 1):P63

Background

Sepsis is a common cause of mortality and morbidity worldwide. Early and appropriate empiric antimicrobial therapy and surgical intervention are critical in decreasing mortality and morbidity. A systematic review by Winters et al suggested that beyond the standard 28-day in-hospital mortality endpoint, ongoing mortality in patients with sepsis remains elevated up to 2 years and beyond[1]. In addition, survivors consistently demonstrate impaired quality of life[2]. The purpose of the study was to evaluate the impact of compliance in 1-year prevention of major adverse events (MAE) in patients with sepsis.

Methods

This is a prospective single center interventional cohort study. We included 82 symptomatic patients (49 male and 33 female) of $57 \pm$ 9.2 years old with sepsis undergoing surgical treatment for purulentnecrotic complications of lower limb from January to December 2013 in Tashkent Medical Academy. Patients were examined every day at the hospital, and every month after discharge during 1-year follow-up. Patients' consent was received at day of admission to the Academy for diagnosis, in-patient treatment and 1-year follow-up examination.

Results

Baseline patients' characteristics are shown in Table 17. During the examination period $28(34.1 \%)$ patients strictly comply with the prescribed antibacterial, antifungal treatment, life style changes (stop smoking, alcohol intake), treatment of comorbidities (arterial hypertension, coronary heart disease, diabetes and al.). 54/82 patients (65.8\%) were non-compliant. In 46 patients (56\%), blood culture was positive. Staphylococcus species were detected in $18(39.1 \%)$, Streptococcus species $9(19.5 \%)$, Enterococcus species in $3(6.5 \%)$, Klebsiella species in $12(21.4 \%)$, E. coli in 8 (14.2\%), Pseudomonas aeruginosa in 11 (23.9\%) patients. Candida species were the most frequent fungi that cause sepsis in 27 patients (58.6\%). During 1 year examination, we detected significant decrease of new admissions for inpatient treatment for purulent surgical complications, MAE, such as cardiac death and myocardial infarction, pulmonary embolism, $\mathrm{p}<0.05$ (Table 18). Kaplan-Meier curve of survival after death is shown in Fig. 38.

Conclusions

Patients with good compliance showed twofold decrease risk for MAE. Compliance initiatives are vitally significant in septic patients to decrease the risk of reactivation of infection, purulent-necrotic complications, cardiac death, and pulmonary embolism.

\section{References}

1. Winters BD, Eberlein M, Leung J, Needham DM, Pronovost PJ, Sevransky JE. Long-term mortality and quality of life in sepsis: a systematic review. Crit Care Med. 2010 May. 38(5):1276-83.

2. Sarikonda KV, Micek ST, Doherty JA, et al. Methicillin-resistant Staphylococcus aureus nasal colonization is a poor predictor of intensive care unit-acquired methicillin-resistant Staphylococcus aureus infections requiring antibiotic treatment. Crit Care Med. 2010 Oct. 38(10):1991-5.

Table 17 (abstract P63) Clinical characteristic of compliant and noncompliant patients with sepsis

\begin{tabular}{lll}
\hline Parameters, $\mathrm{n}(\%)$ & $\begin{array}{l}\text { Compliant } \\
\mathrm{n}=28(100 \%)\end{array}$ & $\begin{array}{l}\text { Non-compliant } \\
\mathrm{n}=54(100 \%)\end{array}$ \\
\hline Skin abscess & $9(32.1)$ & $14(25.9)$ \\
Skin cellulitis (phlegmon) & $19(67.8)$ & $30(74.1)$ \\
Necrotizing fasciitis & $12(42.9)$ & $25(46.3)$ \\
Smoking after operation & - & $42(77.8)$ \\
Type 2 diabetes & $18(64.3)$ & $37(68.5)$ \\
Arterial hypertension in anamnesis & $16(57.1)$ & $29(53.7)$ \\
Arterial hypotension ('90 mmHg) at admission & $28(100)$ & $54(100)$ \\
Coronary artery disease & $26(92.8)$ & $47(87.0)$ \\
Atrial fibrillation & $7(25.0)$ & $9(16.6)$ \\
Supraventricular arrythmia & $8(28.5)$ & $12(22.2)$ \\
Ventricular arrythmia & $5(28)$ & $17(31.5)$ \\
Congestive heart failure & $16(57.1)$ & $38(64.8)$ \\
\hline
\end{tabular}


Table 18 (abstract P63) Clinical outcomes during 1-year postoperative period of patients with skin infection complicated by sepsis

\begin{tabular}{llll}
\hline Clinical outcomes & $\begin{array}{l}\text { Compliant } \\
\mathrm{n}=28(100 \%)\end{array}$ & $\begin{array}{l}\text { Non-compliant } \\
\mathrm{n}=54(100 \%)\end{array}$ & $\mathrm{p}$-value \\
\hline 1 year postoperative mortality & $3(10.7)$ & $14(25.9)$ & 0.033 \\
Myocardial infarction/fatal & $2(7.1) / 1(3.8)$ & $9(16.6) /(7.4)$ & $0.001 / 0.081$ \\
$\begin{array}{l}\text { Fatal acute cardiovascular } \\
\text { insufficiency }\end{array}$ & $2(7.1 \%)$ & $12(22.2)$ & 0.0091 \\
$\begin{array}{l}\text { Ischemic stroke/fatal } \\
\text { Fatal pulmonary embolism }\end{array}$ & - & $4(7.4) / 2(3.7)$ & $0.0612 / 0.3605$ \\
Gastrointestinal bleeding & $1(3.5)$ & $5(9.2)$ & 0.024 \\
Purulent-necrotic complications & $7(25.0)$ & $5(11.1)$ & 0.0718 \\
\hline
\end{tabular}

Kaplan-Meier curve for event-free survival from death in patients with purulent- necrotic complications of lower limb

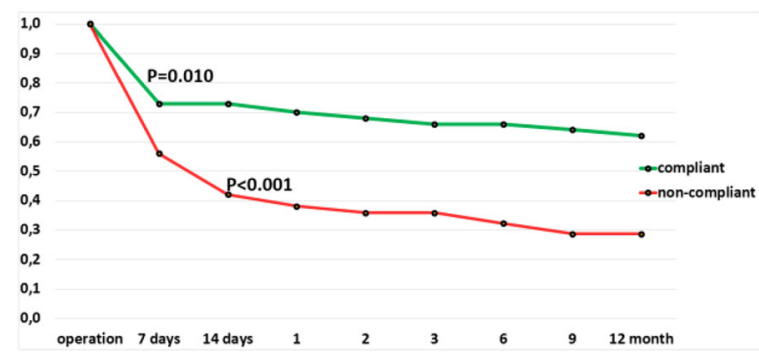

Fig. 38 (abstract P63). See text for description

\section{P64}

Foot synovial tendon complex injury as an important factor of sepsis development and severity of purulent-necrotic complications in type 2 diabetic patients

Aleksandra V. Shalaeva, Evgeniya V. Shalaeva, Bakhtiyor B. Janabaev, Azam R. Bobabekov

Tashkent Medical Academy, Republican Center of purulent surgery and complications of diabetes, Tashkent, Uzbekistan

Correspondence: Aleksandra Shalaeva (aleksandrashalaeva@gmail.com) Intensive Care Medicine Experimental 2017, 5(Suppl 1):P64

\section{Background}

Type 2 diabetic (T2D) patients with the lower extremity artery disease are at the highest risk of lower limbs purulent-necrotic complications and sepsis. The aim of the study was to evaluate the prediction of the synovial tendon complex injury (STCl) on the incidence of septic complications in T2D patients.

Material and methods

In this prospective single center interventional cohort study, we evaluated 179/331 consecutive symptomatic T2D patients (mean age $60.4 \pm 8.8$ years) without a history of cardiac events undergoing trans-femoral amputation (TFA) during the year 2013, who had no contraindications for computed tomographic angiography (CTA). Laboratory tests were performed at the baseline and in dynamics. Patients' consent was received at day of admission to the Academy for diagnosis, in-patient treatment and follow-up examination.
Results

Baseline clinical characteristics are summarized in Table 19. Laboratory and instrumental tests in the different clinical severity of sepsis are shown in Table 20. The incidence of all dorsal and plantar STCI on admission in our Centre in 2013 was similar (52.6\%/47.3\%). Among 179 T2D patients included in this study cohort, 127 (70.95\%) had plantar STCl vs. $52(29.05 \%)$ dorsal. The odds ratio of TFA from plantar complications was $2.65(p=$ 0.0267 ). Severe sepsis and septic shock were developed significantly more often in patients with plantar STCl, $\mathrm{F}(1,177)=99.59, \mathrm{p}<0.001, \mathrm{t}(177)=9.88$ $p<0.001$. The $95 \%$ confidence interval for the slope was .18 to .32 . The correlation coefficient was $0.62, p<0.001$. Figure 39 shows dorsal synovial injury, Fig. 40 indicates the plantar spread of infection. On CTA stenosis $>50 \%$ of the posterior tibial artery was detected in 1.9 times more often than anterior tibial artery $25.6 \%$ vs. $32.2 \%$; occlusion 3.8 times higher $70.4 \%$ vs. $18.4 \%$, respectively $(p<0.001)$. The correlation coefficient between plantar STCl and lesions of the posterior tibial artery was $r=0.71, p<0.001$.

Table 19 (abstract P64) Baseline characteristics and laboratory findings of 179 study patients with type 2 diabetes undergoing trans-femoral amputation of lower limb

\begin{tabular}{|c|c|c|c|c|c|c|}
\hline \multirow[t]{2}{*}{ Parameters } & \multicolumn{2}{|c|}{ Total, $n=179$} & \multicolumn{2}{|c|}{ Male, $n=120$} & \multicolumn{2}{|c|}{ Female, $n=59$} \\
\hline & $\mathrm{n}$ & $\%$ & $n$ & $\%$ & $n$ & $\%$ \\
\hline Arterial hypertension & 161 & 89.9 & 108 & 90.0 & 53 & 89.8 \\
\hline Hyperlipidemia & 179 & 100,0 & 120 & 100,0 & 59 & 100,0 \\
\hline Statin use & 35 & 19.6 & 21 & 17.5 & 14 & 23.7 \\
\hline Current smoking & 99 & 55,3 & 99 & 82,5 & 0 & 0,0 \\
\hline Family history of CAD & 140 & 78,2 & 81 & 67,5 & 59 & 100,0 \\
\hline Clinical CAD & 179 & 100 & 120 & 100 & 59 & 100,0 \\
\hline Congestive heart failure & 133 & 74.3 & 74 & 61.7 & 59 & 100.0 \\
\hline Septicemia & 50 & 27.9 & 28 & 23.3 & 22 & 37.3 \\
\hline Sepsis & 92 & 51.4 & 69 & 57.5 & 23 & 39.0 \\
\hline Severe sepsis & 28 & 15.6 & 18 & 15.0 & 10 & 16.9 \\
\hline Septic Shock & 9 & 5.0 & 5 & 4.2 & 4 & 6.8 \\
\hline
\end{tabular}

CAD coronary artery disease, FC functional class, NYHA New York

Heart Association

Table 20 (abstract P64) Laboratory and instrumental tests in Type 2 diabetic patients underwent trans-femoral amputation of lower limb

\begin{tabular}{llllll}
\hline Data & Septicemia & Sepsis & $\begin{array}{l}\text { Severe } \\
\text { sepsis }\end{array}$ & $\begin{array}{l}\text { Septic } \\
\text { shock }\end{array}$ & $\begin{array}{l}\text { P- } \\
\text { value }\end{array}$ \\
\hline Systolic blood pressure & $158.7 \pm$ & $154.1 \pm$ & $88.7 \pm 9.4^{* \wedge}$ & $64.1 \pm 7.8^{*}$ & $\leq 0.001$ \\
& $14.4 \wedge$ & $22.8 \wedge$ & & & \\
Diastolic blood pressure & $93.4 \pm 5.3 \wedge$ & $91.9 \pm 8.6 \wedge$ & $63.4 \pm 5.3^{* \wedge}$ & $31.9 \pm 7.6^{*}$ & $\leq 0.01$ \\
LVEF, \% & $54.3 \pm 3.3 \wedge$ & $48.7 \pm 4.1^{* \wedge}$ & $43.3 \pm 4.4^{* \wedge}$ & $38.3 \pm 3.1^{*}$ & $\leq 0.05$ \\
HbA1C, \% & $9.1 \pm 1.1 \wedge$ & $13.9 \pm 0.5^{*}$ & $14.4 \pm 2.5^{*}$ & $13.9 \pm 0.9^{*}$ & $\leq 0.05$ \\
Fibrinogen, mg\% & $511 \pm 152 \wedge$ & $722 \pm 132^{*}$ & $754 \pm 127^{*}$ & $995 \pm 165^{*}$ & $\leq 0.001$ \\
Blood urea, mmol/l & $7.1 \pm 1.9 \wedge$ & $12.4 \pm 1.6^{*}$ & $12.1 \pm 2.2^{*}$ & $16.8 \pm 2.7^{*}$ & $\leq 0.001$ \\
Blood creatinine, mmol/ & $88 \pm 18 \wedge$ & $123 \pm 27^{* \wedge}$ & $167 \pm 17^{*}$ & $163 \pm 47^{*}$ & $\leq 0.001$ \\
I & & & & & \\
Brain natriuretic peptide, & $232 \pm 28 \wedge$ & $395 \pm 35^{*}$ & $412 \pm 32^{*}$ & $445 \pm 41^{*}$ & $\leq 0.001$ \\
ng/ml & & & & & \\
\hline
\end{tabular}

* - comparison with septicemia, $\wedge$ - comparison with septic shock 


\section{Conclusion}

Plantar STCl contrast to dorsal, more often complicated by spread of infection and sepsis. We found a strong association between posterior tibial artery lesions and plantar STCl, as well as the development of severe septic complications.

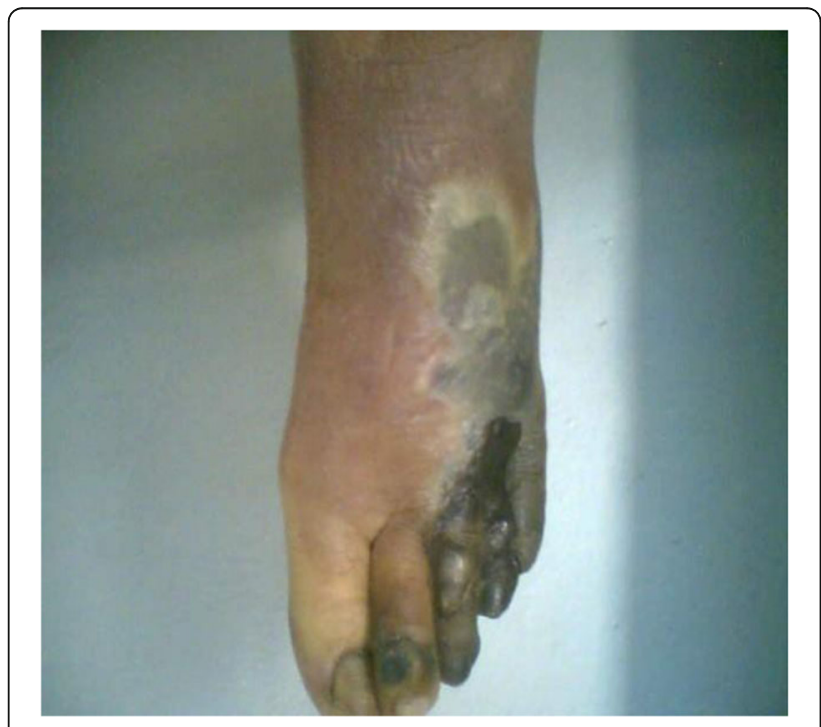

Fig. 39 (abstract P64). Dorsal tendon synovial complex injury

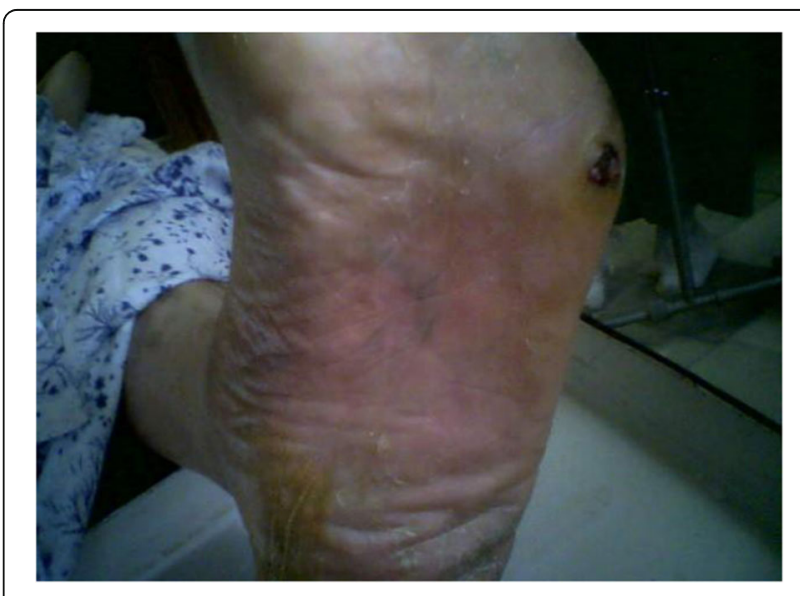

Fig. 40 (abstract P64). Plantar tendon synovial complex injury

\section{Publisher's Note}

Springer Nature remains neutral with regard to jurisdictional claims in published maps and institutional affiliations. 